\title{
Design and Construction of a Compact, Modular Fusion Testbed and Proton Source
}

\author{
Aaron English \\ A thesis submitted to the Faculty of Graduate and Post Doctoral Affairs \\ in partial fulfillment of the requirements for the degree of
}

Master of Applied Science

in

Electrical and Computer Engineering

\author{
Carleton University \\ Ottawa, Ontario, Canada \\ C)2018 Aaron English
}




\begin{abstract}
Design and construction of a Penning Ion Generator (PIG) plasma source with an Einzel focusing lens and a test bed for fusion experiments was undertaken. The unloaded vacuum chamber achieved a clean vacuum with an ultimate pressure of $4 \times 10^{-8}$ Torr and a background gas of $\mathrm{CO}$ and $\mathrm{H}_{2} \mathrm{O}$ as measured by an Residual Gas Analyzer (RGA). The plasma source operated with both argon and nitrogen feed gasses and a maximum current of $2.9 \mu \mathrm{A}$ at an acceleration potential of approximately $5 \mathrm{kV}$ was achieved.
\end{abstract}




\section{Acknowledgements}

Firstly, I would like to thank Tony Tapp, Dr. Walter and Martha Chudobiak, and Dottie Strolovitch for their generous financial contributions that made all of this possible. I would also like to express my gratitude to the wonderful staff at Carleton University, Alex, Angela, Kevin, Nagui, Rob, and Steve for all their technical support, considerate donation of time and expertise which has enabled me to carry out much of this work. I would like to thank my supervisor Steve McGarry for his guidance. His dedication to quality of research, and commitment to genuine and meaningful scientific investigation are a continual source of inspiration and provide an often much needed grounding and focus. Finally, I would like to thank my family, friends, girlfriend, and especially, my parents. Without them I would be surely be adrift in a sea of shenanigans and meaningless noise. 
For Tony, Dottie, Kit, and Stan 


\section{Contents}

Title Page

Abstract

Acknowledgements $\quad$ iii

Dedication $\quad$ iv

Acronyms

$\begin{array}{ll}\text { Constants } & \text { xvi }\end{array}$

Symbols $\quad$ xvii

1 Introduction 1

1.1 Motivation ........................... 1

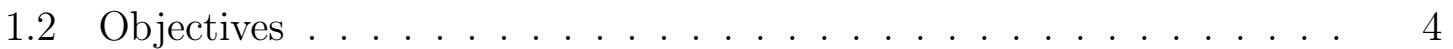

2 Theory and Background $\quad 6$

2.1 Vacuum Theory ..................... 6

2.1.1 Vacuum Considerations . . . . . . . . . . . . . . . . . 8

2.1.2 Formation of the Vacuum _. . . . . . . . . . . . . . . 9

2.1.3 Inspection of the Vacuum . . . . . . . . . . . . . . . . . 10

2.2 Plasmas . . . . . . . . . . . . . . . . . . . . . . . . . 11

2.2.1 Ionization . . . . . . . . . . . . . . . . . 11

2.2.2 Plasma Basics . . . . . . . . . . . . . . . . . . . . . 14

2.2.3 Plasma Sources . . . . . . . . . . . . . . . . . . . . 16

2.2.4 Penning Ion Source . . . . . . . . . . . . . . . . . . 18

2.3 Ion Beams . . . . . . . . . . . . . . . . . . . . . . . . . . . . 19

2.3.1 Extraction From Plasmas . . . . . . . . . . . . . . . 19 
2.3.2 Manipulation and Control of Ions and Ion Beams . . . . . . . 21

2.4 Fusion ............................ 25

2.4.1 Calculating Energy Release . . . . . . . . . . . . 25

2.4.2 The Fusion Process . . . . . . . . . . . . . . . . . . . . . . . 29

3 State of the Art 33

3.1 Fusion Experiments . . . . . . . . . . . . . . . . . . 33

3.2 Small Scale Fusion Experiments . . . . . . . . . . . . . . . . . 34

3.2.1 Pyroelectric Fusion . . . . . . . . . . . . . . . . . 35

3.2.2 Plasma Source . . . . . . . . . . . . . . . . . 35

3.3 Previous Experimental System and its Limitations . . . . . . . . . . . 36

4 Design $\quad 38$

4.1 Initial Considerations . . . . . . . . . . . . . . . . . . 38

4.2 Target Sub-chamber . . . . . . . . . . . . . . . . 42

4.3 Ion Source and Electrostatic Lenses . . . . . . . . . . . . . . . . . 44

4.3.1 Theoretical Design of the Ion Source . . . . . . . . . . . . . . 44

4.3.2 Theoretical Design of the Electrostatic Lenses . . . . . . . . . 46

4.3.3 Simulation of Beam Extraction and Optics . . . . . . . . . . 47

4.4 Final System Design Parameters . . . . . . . . . . . . . . . . 59

5 Construction and Experimental Results $\quad 61$

5.1 Construction of Vacuum System . . . . . . . . . . . . . . . 61

5.2 Vacuum System Results . . . . . . . . . . . . . . . . . . 64

5.3 Construction of Internal Components . . . . . . . . . . . . . . 67

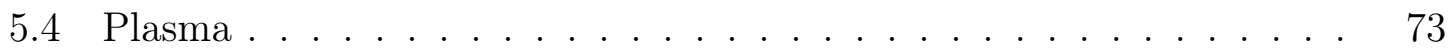

5.4.1 Nitrogen Plasma ................. . . 73

5.4.2 Argon Plasma .................... 76 
$\begin{array}{lll}6 & \text { Discussion } & 79\end{array}$

6.1 Pressure Management . . . . . . . . . . . . . . . . 79

6.2 Resistor Array Arcing . . . . . . . . . . . . . . . 80

6.3 Vacuum Arcing . . . . . . . . . . . . . . . 80

$\begin{array}{lll}7 & \text { Conclusions } & 81\end{array}$

7.1 Recommendations and Future Work . . . . . . . . . . . . . 81

7.1.1 Chamber.................... 81

7.1 .2 Simulation ....................... 82

7.1.3 Penning Ion Generator . . . . . . . . . . . . . . 82

$\begin{array}{lr}\text { References } & 84\end{array}$

$\begin{array}{ll}\text { Glossary } & 89\end{array}$ 


\section{List of Figures}

1 World TPES (of 158.7 PW h total) by fuel type for 2015 . . . . . . . . 1

2 World TFC (of $109.1 \mathrm{PW}$ h total) by fuel type for 2015 . . . . . . . . 2

3 Comparison of total global electricity production and oil consumption 3

4 An oversized cutaway showing the $\mathrm{CF}$ compression seal . . . . . . . . 9

5 Impact ionization cross section for $\mathrm{H}_{2}, \mathrm{H}_{2}^{+}$, and $\mathrm{H}$. . . . . . . . . 13

6 Sketch of PIG basic design . . . . . . . . . . . . . . . . . 18

7 Diagram showing the Pierce angle on the extraction electrode (blue) as applied to an extracted electron beam (red) with direction of travel indicated by the arrow . . . . . . . . . . . . . . . 20

8 Sketches of emittance ellipses and interpretation of particle behaviour 25

$9 \quad$ A visualization of the possible fusion pathways for ${ }_{1}^{2} \mathrm{H}-{ }_{1}^{2} \mathrm{H}$ fusion. . . 27

10 Binding energy per nucleon of stable nucleons . . . . . . . . . . . 28

11 Sketch of the approximate potential well of a nucleus . . . . . . . . 30

12 Cut away of the original pyroelectric based accelerator and vacuum chamber ....................... . . . . . 37

13 Base pieces for modular test bench . . . . . . . . . . . . . . . 38

14 Base beamline showing key components . . . . . . . . . . . . . . . . . 40

15 Full assembly CAD design . . . . . . . . . . . . . . . . 41

16 The target sub-chamber . . . . . . . . . . . . . . . 42

17 Cutaway of the beamline with the target sub-chamber in place demonstrating clearance . . . . . . . . . . . . . . . . . . 43

18 Results of COMSOL magneto-static simulations $\left(\log \left(\frac{B}{1 \mathrm{~T}}\right)\right) \quad \ldots \quad \ldots \quad 45$

19 CAD design of PIG source . . . . . . . . . . . . . 46

20 CAD design of PIG with Einzel lens and mounting flange . . . . . . . 47 
21 Comparison of extraction angles and visualization of meniscus potential where the $z$-axis is the direction of the ion beam travel, and the $x$-axis is the distance from the center of the beam. The system is nearly axially symmetric about $z \ldots \ldots . \ldots 53$

22 Demonstration of flattening of meniscus equipotential . . . . . . . . 54

23 Emittance plots for the extracted beam taken between the first Einzel lens electrode and the plasma electrode; where $y$ is the distance from the beam center, travelling along the $z$-axis. The system is nearly axially symmetric about $z \ldots \ldots \ldots \ldots$

24 Full beamline simulations with no Einzel lens, one extraction electrode, and Einzel lenses with different extraction angles to show trajectories. The $x$-axis is the distance to the center of the beam and the $z$-axis is the direction of travel for the ion beam. The system is nearly axially symmetric about $z \ldots \ldots \ldots \ldots$. . . . . . . . . . . 56

25 Full beamline simulations to show trajectories . . . . . . . . . 57

26 Demonstration of beam characteristics after passing through the Einzel lens, $x$ and $y$ are distances to the center of the beam travelling along the $z$-axis. Emittance plot taken after the Einzel lens. . . . . . . . 58

27 Top-down view of slice of beamline with major component dimensions. All values are in centimeters . . . . . . . . . . . . . . . . 59

28 Construction of the frame . . . . . . . . . . . . . . . . 61

29 Assembling the chamber before lowering it into place . . . . . . . . . 62

30 Chamber positioned on frame . . . . . . . . . . . . 63

31 Completed vacuum chamber and pump, sealed for initial vacuum testing 64

32 RGA results after the first pump down . . . . . . . . . . . . 65

33 RGA results after a multi-day pump down . . . . . . . . . . 65

34 RGA results after successful sealing of all leaks . . . . . . . . . . . 66 
35 Pressure of the chamber 24 hours after application of epoxy to leak . 66

36 RGA results under static vacuum . . . . . . . . . . . . . 67

37 Manufactured Components for PIG and Einzel Lens . . . . . . . . . . 68

38 CNC Milled target sub-chamber . . . . . . . . . . . . . . 68

39 Assembly of PIG and Einzel Lens . . . . . . . . . . . . . . . . 69

40 Electrical schematic of system . . . . . . . . . . . . . . . 70

41 Mounted and electrically connected target sub-chamber . . . . . . . . 70

42 Mounted, electrically connected, and gas connected PIG with Einzel lens 71

43 External electrical connection for system . . . . . . . . . . . 72

44 Image of nitrogen plasma and extracted beam . . . . . . . . . . . 74

45 Optical Spectrum of nitrogen plasma . . . . . . . . . . . . 75

46 RGA spectrum for nitrogen plasma trials . . . . . . . . . . . . . . 75

47 Image of argon plasma and extracted ions . . . . . . . . . . . . 76

48 Optical spectrum of argon plasma . . . . . . . . . . . . . . 77

49 RGA spectrum for argon plasma trials . . . . . . . . . . . . . 78

50 Flow chart for beamline simulation code . . . . . . . . . . . . . . 99 


\section{List of Tables}

1 Vacuum Levels, Pressure Ranges, and Key Details . . . . . . . . . . . 6

2 Diameter $\left(D_{m}\right)$ of Some Typical Gasses . . . . . . . . . . . . . 8

3 Channel Peaks and Their Relative Intensity for Important Gasses . . 11

4 Commonly used coefficients for binding energy calculation . . . . . . 26

$5 \quad$ Fundamental forces and their basic properties . . . . . . . . . . . 29

$6 \quad$ Important Reaction in Nuclear Fusion . . . . . . . . . . . . . . . . . . 31

7 Tabulated currents for different extraction geometries . . . . . . . . 51

8 Tabulated currents for different extraction geometries . . . . . . . . . 52

$9 \quad$ Required separation of components as determined by the simulations $\quad 59$

10 Electrode Potentials . . . . . . . . . . . . . . . . . 60 


\section{List of Appendices}

Appendix A Code ......................... 93

A.1 Beamline Simulation Suite README . . . . . . . . . . . . . 93

A.1.1 Introduction . . . . . . . . . . . . . . . . . . 93

A.1.2 Required Packages . . . . . . . . . . . . . . . 93

A.1.3 Installation and Setup Process . . . . . . . . . . . . 94

A.1.4 Code Hierarchy . . . . . . . . . . . . . . . . . . . . . 98

A.1.5 Usage . . . . . . . . . . . . . . . . 100

A.2 Beamline Simulation Code . . . . . . . . . . . . . . 102

A.2.1 multiRunPy . . . . . . . . . . . . . . . . . . 102

A.2.2 $\operatorname{runObj} \ldots \ldots \ldots \ldots \ldots \ldots$

A.2.3 addTools . . . . . . . . . . . . . . . 106

A.2.4 geomPrep . . . . . . . . . . . . . . . 107

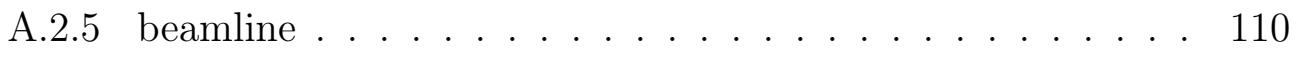

A.2.6 beamDiag . . . . . . . . . . . . . . . 117

A.2.7 analysis . . . . . . . . . . . . . . 122

A.3 Additional Calculation Code . . . . . . . . . . . . . . . . 124

A.3.1 simParamCalc . . . . . . . . . . . . . . . . . . 124

A.3.2 wallThickCalc . . . . . . . . . . . 126

A.3.3 geomPrepManDef . . . . . . . . . . . . . 126 


\section{Acronyms}

304-SS Type 304 Stainless Steel. 8, 45, 61, 67, 89, Glossary: 304-SS

316-SS Type 316 Stainless Steel. 8, 61, 89, Glossary: 316-SS

CAD Computer Aided Design. viii, 38, 41, 42, 46, 47, 91

CERN European Organization for Nuclear Research. 90, Glossary: CERN

CF Conflat Flange. viii, 8, 9, 38, 39, 65, Glossary: CF

CLI Command Line Interface. 97, 98

CNC Computer Numerically Controlled. x, 68

COMSOL COMSOL Multiphysics (COMSOL). viii, 44, 45, 47, 48, Glossary: COMSOL

CT Compact Toroid. 17

DC Direct Current. 16-19

ECR Electron Cyclotron Resonance. 17

FE Field Emission. 18, 83

FRC Field-Reversed Configuration. 17

GF General Fusion. 34

GHG Greenhouse Gas. 1-3, Glossary: GHG

HV High Vacuum. 6, 8, 9, 38, 89, Glossary: HV

IC Inertial Confinement. 33, 34 
ISO International Organization for Standardization. 92, Glossary: ISO

ITER International Tokamak Experimental Reactor. 33

JANIS Java-base Nuclear Data Information System. 30, Glossary: JANIS

MC Magnetic Confinement. 33, 34

MFP Mean Free Path. 7, 39, 79, Glossary: MFP

NdFeB Neodymium Iron Boron Rare Earth Permanent Magnet. 44

NEA Nuclear Energy Agency. 30, 90, Glossary: NEA

NIF National Ignition Facility. 33

OECD Organization for Economic Co-operation and Development. 91, Glossary: OECD

PIG Penning Ion Generator. ii, viii, x, 17-19, 36, 44-47, 59, 67-69, 71, 73, 79, 81-83, Glossary: PIG

PTFE Polytetraflouroethylene (PTFE), commonly referred to by the brand name Teflon, is a synthetic polymer with highly desirable electrical breakdown characteristics. 82

RF Radio Frequency. 10, 16, 17, 19, 21

RGA Residual Gas Analyzer. ii, ix, x, 10, 11, 64-67, 73, 75, 77, 78, Glossary: RGA

SAE SAE International. 89, Glossary: SAE

SmCo Samarium Cobalt Rare Earth Permanent Magnet. 44

SQL Structure Query Language. 48, 91, Glossary: SQL 
TFC Total Final Consumption. viii, 1, 2, Glossary: TFC

TPES Total Primary Energy Supply (TPES). viii, 1, Glossary: TPES

UHV Ultra High Vacuum. 6, 8, 9, 89, 90, Glossary: UHV 


\section{Constants}

$R_{y} \quad$ Rydberg energy $(13.605693 \mathrm{eV})$

$\varepsilon_{0}$ permittivity of free space $\left(8.854187 \times 10^{-12} \mathrm{~F} \mathrm{~m}^{-1}\right)$

$a_{0}$ Bohr radius $\left(5.291772 \times 10^{-11} \mathrm{~m}\right)$

c speed of light $\left(2.997924 \times 10^{8} \mathrm{~m} \mathrm{~s}^{-1}\right)$

$e$ elementary charge $\left(1.602176 \times 10^{-19} \mathrm{C}\right)$

$h$ Planck constant $\left(6.626070 \times 10^{-34} \mathrm{~m}^{2} \mathrm{~kg} \mathrm{~s}^{-1}\right)$

$k$ Boltzmann constant $\left(1.380648 \times 10^{-23} \mathrm{~J} \mathrm{~K}^{-1}\right)$

$m_{e}$ mass of electron $\left(9.109383 \times 10^{-31} \mathrm{~kg}\right)$ 


\section{Symbols}

A total number of nucleons in nucleus (unitless). 26

$B_{e}$ electron orbital binding energy $(\mathrm{eV}) .13,14$

$B$ magnetic field (T). 18, 22, 49

$D_{m}$ diameter of gas molecules (m). xi, 7, 8, 39

$E_{B}$ nuclear binding energy $(\mathrm{eV}) .26$

$E_{D}$ mass defect $(\mathrm{eV}) .28$

$E_{k}$ kinetic energy $(\mathrm{eV}) .13,20,21$

$E_{o}$ electron orbital average kinetic energy $(\mathrm{eV}) .13,14$

$E$ energy (J). 4, 12, 26

$J$ current density $\left(\mathrm{A} \mathrm{m}^{-2}\right) .20$

$L_{11}$ mean free path of gas molecules in gas of same type $(\mathrm{m}) .7$

$L_{i 1}$ mean free path of ions in neutral gas of same atomic type (m). 7, 39

$L$ electrode separation (m). 20, 23, 24

$M$ mass of ion (kg). 15, 16, 20, 21

$N_{o e}$ electron occupation number (unitless). 13

$N$ number of neutrons in nucleus (unitless). 26

$P$ pressure $(\mathrm{Pa}) .7,8,39$

$R_{r}$ number of reactions (unitless). 31

$R$ radius of curved trajectory $(\mathrm{m}) .22$ 
$T_{e}$ electron temperature (K). 15, 16, 45

$T$ temperature $(\mathrm{K}) .7,8,39$

$V$ applied voltage (V). 20-24

$Z$ number of protons in nucleus (atomic mass number - unitless). 21, 22, 26

$\Delta E_{B}$ change in nuclear binding energy i.e. energy released in reaction (eV). 27, 28

$\Delta$ pairing energy parameter $(\mathrm{eV}) \cdot 26$

$\Phi$ particle flux $\left(\mathrm{s}^{-1}\right) .31$

$\lambda_{D}$ Debye length (m). 15, 20, 21

$\lambda$ wavelength (m). 12

$\mathcal{E}$ electric field $\left(\mathrm{V} \mathrm{m}^{-1}\right) \cdot 12,23,24,49$

$\nu_{B}$ Bohm velocity $\left(\mathrm{m} \mathrm{s}^{-1}\right) .15$

$\nu$ particle velocity $\left(\mathrm{m} \mathrm{s}^{-1}\right) .15,22,49$

$\phi_{P W}$ plasma potential (V). 16

$\phi_{P}$ potential of presheath region $(\mathrm{V}) .16$

$\phi_{W}$ potential of plasma sheath $(\mathrm{V}) .16$

$\phi$ self-consistent electric potential (V). 49

$\rho$ space-charge $\left(\mathrm{C} \mathrm{m}^{-3}\right) .49$

$\sigma$ interaction cross section $\left(\mathrm{b} / 1 \times 10^{-24} \mathrm{~cm}^{2}\right) .31$

$f$ particle distribution function $\left(\mathrm{C} \mathrm{s} \mathrm{m}^{-4}\right) .49$

$m$ mass $(\mathrm{kg}) .4,22,26,49$ 
$n_{e}$ number density of electrons $\left(\mathrm{m}^{-3}\right) \cdot 15,45$

$n$ number density $\left(\mathrm{cm}^{3}\right) .31$

$q$ charge (C). 22, 49

$r^{\prime}$ slope of the particle trajectory relative to travel axis (unitless). 23,24

$r$ particle distance from travel axis (m). 23, 24

$z$ penetration depth $(\mathrm{cm}) .31$ 


\section{Introduction}

\subsection{Motivation}

In the scientific community, it is no controversial statement to say that humanity faces an existential threat in climate change. The cause of this is well known to be anthropogenic Greenhouse Gasses (GHGs), the biggest component of which is $\mathrm{CO}_{2}$ [1] generated as a by-product from nearly all forms of mechanical work, as well as a significant portion of electrical power generation. An important metric in investigating global energy usage is Total primary energy supply (TPES). This gives a picture of all the energy sources used before their transformation into the form of final use, and so enables inspection that includes loss and use in all forms. This breakdown is shown in Figure 1. [2] This can then be compared against another

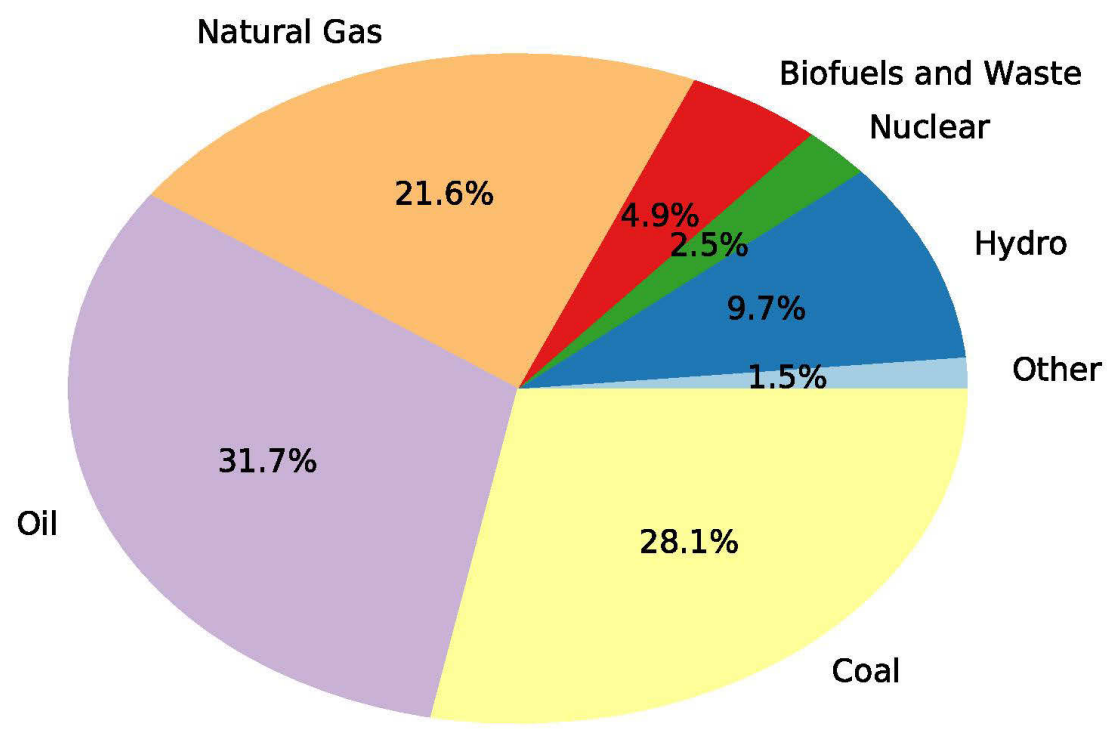

Figure 1: World TPES (of 158.7 PW h total) by fuel type for 2015

metric Total final consumption (TFC) which gives the total fuel consumed in its final form, after conversion to electricity or refinement, shown in Figure 2. As is made 


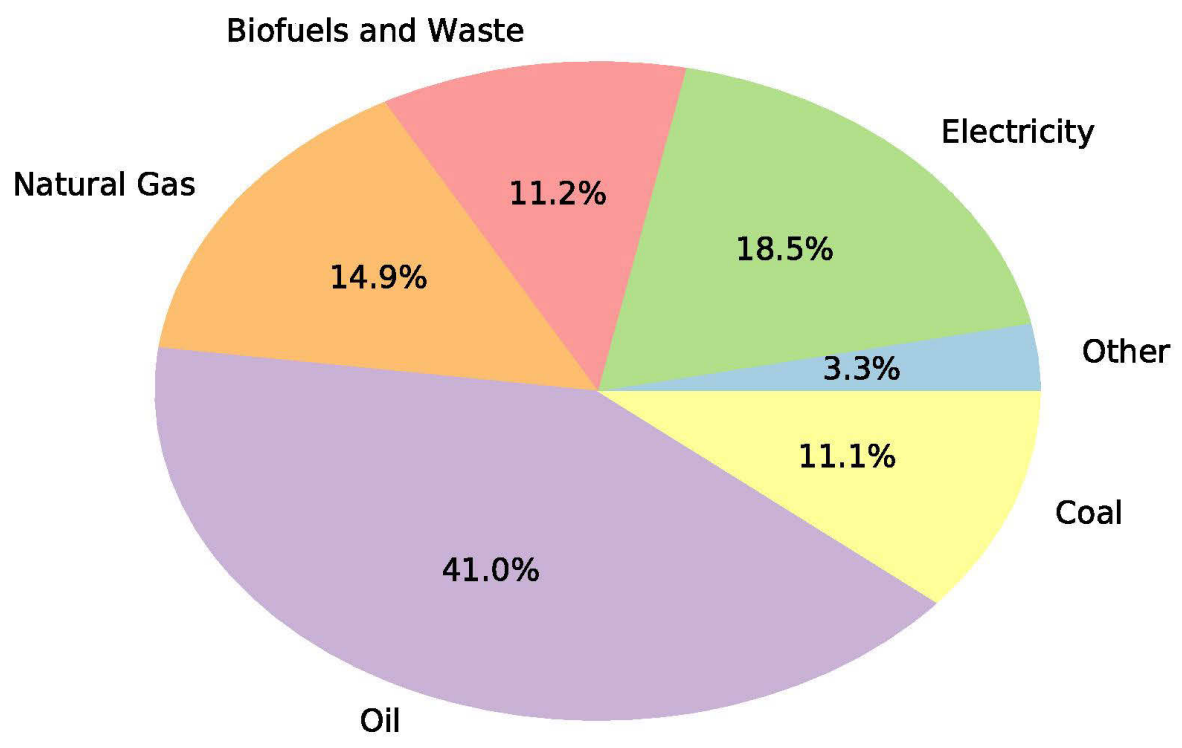

Figure 2: World TFC (of 109.1 PW h total) by fuel type for 2015

clear comparing Figures 1 and 2, $\mathrm{CO}_{2}$ emitting fuels make up $86.7 \%$ of all consumed fuel, and what's more, the vast majority of the fuel's final form is not as electricity, which accounts for only $18.5 \%$ of the worldwide TFC.

Delving into the electricity production by method, and comparing it against the usage of oil alone as done in Figure 3, a more complete picture of the global energy crisis and environmental crisis can be formed. In order to decisively combat climate change without sacrificing technological advances, or condemning the vast majority of the human population to poverty, the amount of fuel consumed that produces GHGs must see an incredible reduction. However, as Figure 3 shows, more than twice as much oil is consumed by road vehicles as is produced in electricity globally (in equivalent units), and in addition to this, only $37.8 \%$ of global energy production is done using clean technologies. Combatting climate change and averting a worldwide energy crisis will require not only increased power generation by clean sources, but a rapid shift from traditional combustion engines as well. The current trend is for combustion engines to be replaced with rechargeable electric vehicles, and while they 


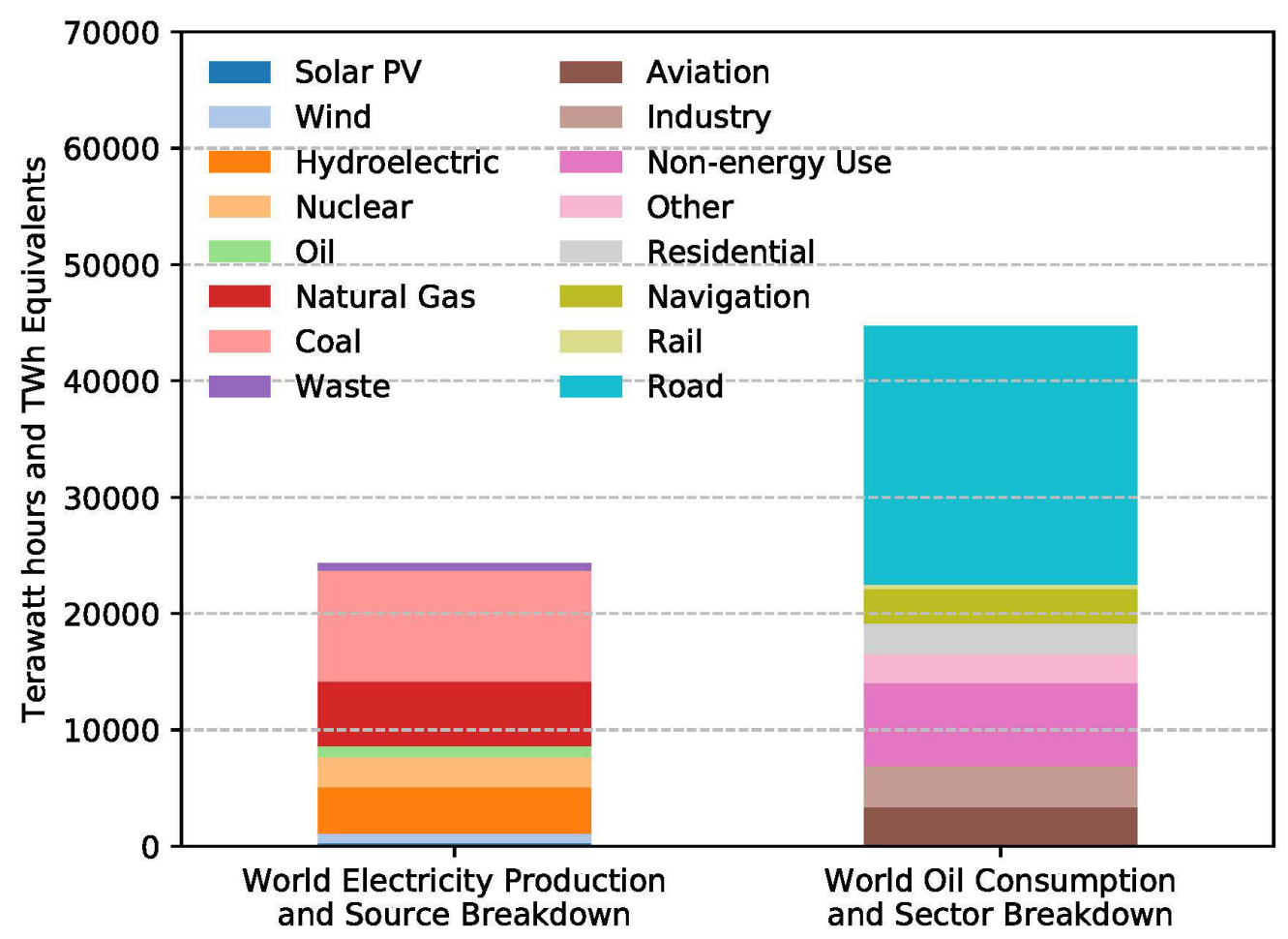

Figure 3: Comparison of total global electricity production and oil consumption

provide dramatically more efficiency in many respects, unless the source of their charging is from clean sources, then the result will be an increase in traditional GHG emitting power plants, negating any benefit and continuing to accelerate climate change.

It quickly becomes apparent that another clean power source is desperately needed. In order to be the difference needed, this power source must have a number of key properties.

1. The power source must have very high power density (for weight and volume restrictions)

2. Its by-products must be easily managed (and preferably be biologically and ecologically inert)

3. Its fuel must be universally available, and cheap. 
The technology that promises to fill all these requirements is nuclear fusion. While thus far a net positive nuclear fusion power source has not been developed, it is notable that under many circumstances it can be achieved, as evidenced by the many groups, individuals, and amateurs (notably including a number of secondary school students) that have successfully attained fusion conditions. [3] Fusion's promise lies in its incredible power density, a result of the direct conversion of mass to energy, which as described by the famous equation $E=m c^{2}$, gives enormous amounts of power. This stands in stark contrast to other clean sources like solar, or wind, where intermittency and density are severely limiting. On the other side of the spectrum, the potential power that could be generated by a fusion power source dwarfs conventional fuels, which rely breaking of chemical bonds to generated energy, which have significantly lower binding energy.

Beyond its promise of power density and output, fusion, is potentially the cleanest form of power, giving off primarily helium as a by produced. When then contrasted with modern nuclear plants, all of which are fission, its promise grows even more. Fusion reactions would be incapable of dangerous run away and not useable as weapons or in the development of them. Fusion power is better in every way and in no ways worse.

\subsection{Objectives}

Given the great potential fusion has, and its underrepresentation in the green energy development sector, it was seen as critical that more effort and focus be put on it. The objective of this work was to contribute to the development of possible fusion power sources by constructing a test bed for investigation of fusion fuels. The scope of the project required the test bed to be flexible and modular to enable growth

and retain enough plasticity to meet the evolving needs of the investigation. As a starting point, to narrow the scope of the project, the test bed was made to be a beam 
target fusion experiment with a proton beam extracted from a plasma ion source. 


\section{Theory and Background}

\subsection{Vacuum Theory}

Fusion experiments, particularly those relying on ion beams, are performed in vacuum environments in order to control atomic species involved in reactions. This is especially true when constructing any sort of ion beam, where the background gas greatly influences the ion path length and beam contaminants. Typically, vacuum is thought of as an absence of particles, but vacuum levels exist on a continuum where the number of particles within a volume decreases, but does not necessarily go to zero. This is an important distinction because even in High Vacuum (HV) or Ultra High Vacuum (UHV), the time or distance between particle interactions, or the ability of the background gas to contribute to contamination is non-zero.

This is exemplified in Table 1, which shows the pressure ranges typically indicated when vacuum levels are discussed, as well as the number of molecules that would be present at such a vacuum level (at room temperature), and the primary constituent gasses that would make up a gas at different levels. [4] Important to understanding vacuum systems, it is common to use units of Torr in vacuum science and systems. Originally defined as 1/760 atm, but now defined as $133.3 \mathrm{~Pa}$, this unit is not frequently used in calculations which are by convention done in SI units, but descriptions and measurements of vacuum systems are typically in Torr. Given this

Table 1: Vacuum Levels, Pressure Ranges, and Key Details

\begin{tabular}{c|c|c|c|c}
$\begin{array}{c}\text { Vacuum } \\
\text { Level }\end{array}$ & $\begin{array}{c}\text { Pressure } \\
(\text { Torr })\end{array}$ & $\begin{array}{c}\text { Pressure } \\
(\mathrm{Pa})\end{array}$ & $\begin{array}{c}\text { Molecular } \\
\text { Density } \\
\left(\mathrm{cm}^{-3}\right)\end{array}$ & $\begin{array}{c}\text { Primary Gas } \\
\text { Composition }\end{array}$ \\
\hline Rough & 760 & $1.01325 \times 10^{5}$ & $3 \times 10^{19}$ & Wet Air \\
\hline High & $1 \times 10^{-3}$ & $1.33 \times 10^{-1}$ & $4 \times 10^{13}$ & Water Vapour \\
\hline Ultra High & $1 \times 10^{-6}$ & $1.33 \times 10^{-4}$ & $4 \times 10^{10}$ & $\mathrm{H}_{2} \mathrm{O}, \mathrm{CO}$ \\
\hline & $1 \times 10^{-8}$ & $1.33 \times 10^{-6}$ & $4 \times 10^{8}$ & $\mathrm{H}_{2} \mathrm{O}, \mathrm{CO}, \mathrm{N}_{2}, \mathrm{H}_{2}$ \\
\hline & $1.33 \times 10^{-8}$ & $4 \times 10^{6}$ & $\mathrm{CO}, \mathrm{H}_{2}$
\end{tabular}


persistence of molecules, a particularly useful measure for many interactions is the Mean Free Path (MFP). This is a distance defined as the average distance a particle will go between interactions. It can be determined using Equation 1 and is of great value in determining necessary vacuum levels. [5]

$$
L_{11}=\frac{k T}{\sqrt{2} \pi D_{m}^{2} P}
$$

Where $k$ is the Boltzmann constant $\left(1.380648 \times 10^{-23} \mathrm{~J} \mathrm{~K}^{-1}\right), T$ is the temperature $(\mathrm{K}), D_{m}$ is the diameter of gas molecules $(\mathrm{m}), P$ is the pressure $(\mathrm{Pa})$, and $L_{11}$ is the mean free path of gas molecules in gas of same type (m). Examining Equation 1 it's clear that the equation is dependent on the molecular diameter $\left(D_{m}\right)$ of the constituent atoms. If, however, the gas is ionized, it is no longer sufficient to assume a $D_{m}$ of the same value, as the size of the atom has changed significantly. The equation thus becomes 2 .

$$
L_{i 1}=\frac{k T}{\pi D_{m}^{2} P}
$$

Where $L_{i 1}$ is the mean free path of ions in neutral gas of same atomic type (m). It should be noted, however, that Equation 2 only accounts for the great difference in velocity of the ions as compared to the atoms of the background gas and does not consider the difference in size of the ions, and of ions of different degrees of ionization, and so is useful only for simple calculations. As an example, Table 2 lists some commonly used gasses, and their molecular diameters for calculations of MFP. $[5]$ 
Table 2: Diameter $\left(D_{m}\right)$ of Some Typical Gasses

\begin{tabular}{c|c|c} 
Gas & $\begin{array}{c}\text { Chemical } \\
\text { Formula }\end{array}$ & $\begin{array}{c}\text { Diameter } \\
(\mathrm{m})\end{array}$ \\
\hline Nitrogen & $\mathrm{N}_{2}$ & $3.74 \times 10^{-10}$ \\
\hline Argon & $\mathrm{Ar}$ & $3.66 \times 10^{-10}$ \\
\hline Hydrogen & $\mathrm{H}_{2}$ & $2.74 \times 10^{-10}$ \\
\hline Oxygen & $\mathrm{O}_{2}$ & $3.59 \times 10^{-10}$
\end{tabular}

\subsubsection{Vacuum Considerations}

Formation and maintenance of the requisite vacuum levels involves appropriate selection of all components being placed in the vacuum, as well as selection of pumps, and sealing faces. A key detail of particular importance is the elimination of leaks, both real and virtual. Fortunately, given the importance of UHV and HV chambers to semiconductor manufacturing and many areas of research, there exist industry standards for vacuum flanges and so a lot of design work can be sidestepped.

Commonly used vacuum flanges are Conflat Flange CF flanges, sexless flanges that work by compressing a soft metal gasket (typically copper) between two knife edges formed of a hard metal like type 304 stainless steel or type 316 stainless steel (316-SS) on both mating surfaces. An oversized cutaway of this seal is shown in Figure 4. This all metal design provides a variety of benefits over a fluorocarbon gasket. Correctly selected metals like stainless steel and copper have much lower permeability to even small gas molecules like hydrogen, and much lower vapour pressures than even other metals.

$$
\log P=\log (101325)\left(A+\frac{B}{T}+C \log T+\frac{D T}{10^{3}}\right)
$$

The vapour pressure of a metal $(P)$ in Pa can be calculated using Equation 3, where $T$ is the temperature $(\mathrm{K})$; and $A, B, C$, and $D$ are fitted coefficients whose value is given in [6]. Using this, the vapour pressure of zinc (a good example of a low vapour pressure metal) can be found. While at room temperature, zinc has a low vapour 


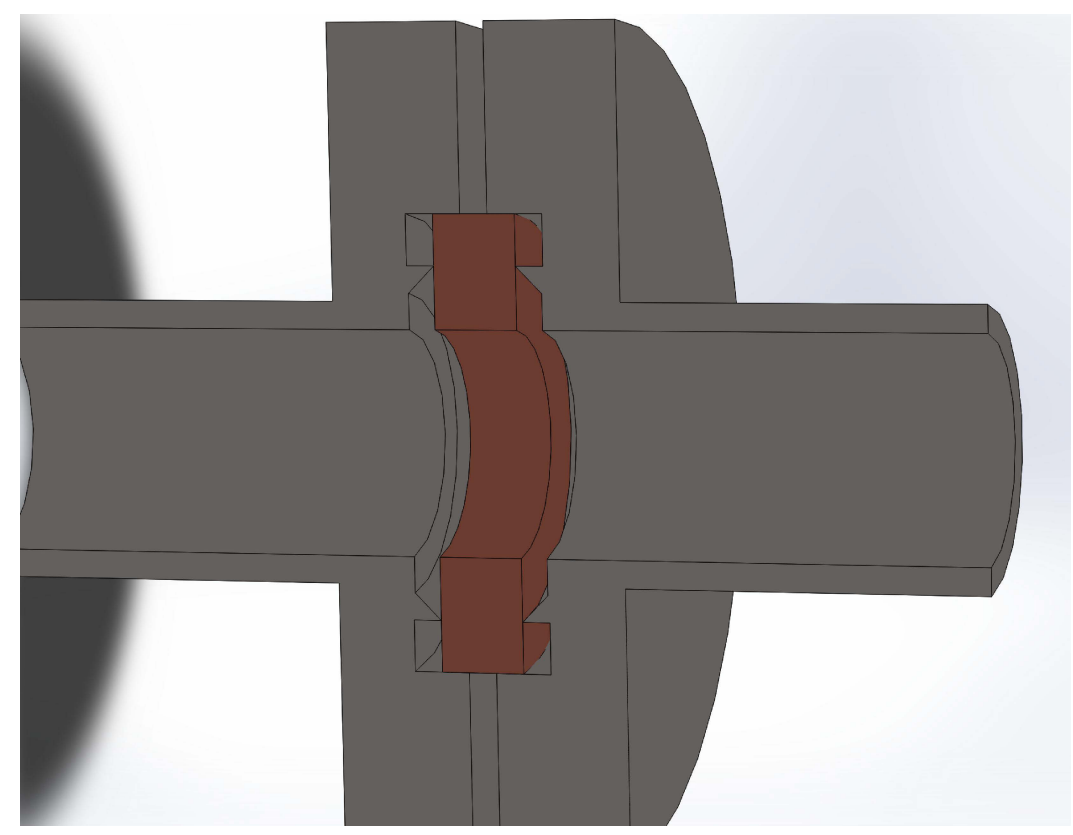

Figure 4: An oversized cutaway showing the CF compression seal

pressure of around $1 \times 10^{-14}$ Torr, but this value rises rapidly and even at $200^{\circ} \mathrm{C}$ the vapour pressure is $1 \times 10^{-5}$ Torr. If used in a high vacuum system where it was exposed to heat, zinc would quickly coat anything within line-of-sight. [7]

In addition to reduced outgassing concerns, all metal flanges enable the system to be baked out while sealed. Baking out is a process by which the entire chamber is heated evenly to force the desorption of gas molecules adsorbed to the inside surfaces of the chamber. The most common molecule adsorbed to the surface of the chamber is water, which without forced desorption, acts as a virtual leak inside the chamber and slowly desorbs, limiting the ultimate attainable pressures of the system. [5]

\subsubsection{Formation of the Vacuum}

With appropriate sealing, HV or UHV levels can be attained using a two or more stage vacuum pump. The first stage, as seen from the vacuum chamber, is the pump used to attain either HV or UHV. These specialized pumps do this in a variety of fashions, but are classified into overarching groups like entrapment pumps, and gas 
transfer pumps. [8] Entrapment pumps typically operate by a form of gas absorption or adsorption to reduce the pressure inside the chamber. Gas transfer pumps, on the other hand, operate as the name suggests by moving gas, and preventing backflow. One of the more common high vacuum pump devices are turbomolecular pumps, typically referred to as turbopumps, which operate by imparting the gas molecules with significant kinetic energy away from the chamber. These are often highly desirable as they can be the lowest maintenance and most cost-effective systems. Turbopumps, however, are limited to higher ultimate pressures than more sophisticated pumps, but can nonetheless achieve pressures greater than $1 \times 10^{-10}$ Torr. These pumps, however, cannot operate at atmospheric pressures and so must be accompanied by a second stage, the backing pump. This stage uses a more conventional vacuum pump, be it a rotary vane pump, or other liquid sealed pumps, or a dry pump. It is frequently advantageous for system operation to use a dry pump, where no pump oil can backflow into the vacuum chamber in failure conditions, as any failures in the system can result in a destructive backflow of pump oil into the chamber.

\subsubsection{Inspection of the Vacuum}

Once constructed, the quality of a vacuum chamber's seal can be inspected using an Residual Gas Analyzer (RGA), and compared against the expected species for a vacuum system as described in Table 1 . The RGA is a specialized mass spectrometer used in identifying species that make up a gas. RGAs typically use a quadrupole array, operated at Radio Frequency (RF) frequencies to discriminate masses. As the quadrupoles oscillate, the changing electric fields cause the ions travelling down the array to oscillate as well. This oscillation is dependent on the charge to mass ratio of the ion, and by sweeping the frequency, the particles whose mass allows them to travel down the quadrupole array to the sensor can be varied, allowing for the detection of a wide range of molecules. Table 3 shows the relative intensity of mass channels peaks 
for important gasses. [8] These mass channels correspond directly to the molecular mass of the fragment, and so is the same for all RGAs If after analysis of a RGA

Table 3: Channel Peaks and Their Relative Intensity for Important Gasses

\begin{tabular}{|c|c|c|c|c|c|c|c|c|}
\hline $\begin{array}{l}\text { Mass } \\
\text { Channel } \\
\text { (Molecular Mass) }\end{array}$ & $\mathbf{H}_{\mathbf{2}}$ & $\mathbf{H e}$ & $\mathbf{H}_{\mathbf{2}} \mathbf{O}$ & $\mathbf{N}_{\mathbf{2}}$ & $\mathbf{O}_{\mathbf{2}}$ & $\mathrm{Ar}$ & $\mathrm{CO}_{\mathbf{2}}$ & $\mathbf{C O}$ \\
\hline 2 & 100 & & & & & & & \\
\hline 4 & & 100 & & & & & & \\
\hline 12 & & & & 7 & & & & \\
\hline 14 & & & & & 11 & & 9 & \\
\hline 16 & & & 25 & & & & & \\
\hline 17 & & & 100 & & & & & \\
\hline 20 & & & & & & 10 & & \\
\hline 28 & & & & 100 & & & 11 & 100 \\
\hline 32 & & & & & 16 & & & \\
\hline 40 & & & & & & 100 & & \\
\hline 44 & & & & & & & 100 & \\
\hline
\end{tabular}

spectrum a real leak is suspected, the RGA can be used to perform a helium leak test. A helium leak test takes advantage of the helium atom's small size to identify locations of leaks by using a wand with a flow controlled helium source at the end. This wand is moved around the chamber until the helium is close enough to the leak that it can penetrate through the crack and subsequently be detected by the RGA. Given the very low atmospheric concentration of helium, a helium source near a leak creates a very distinct signature; and with an RGA set to scan exclusively for the helium mass, this test can be extremely effective in identifying even very slow leaks.

\subsection{Plasmas}

\subsubsection{Ionization}

A cornerstone of understanding plasmas is ionization. Ionization is a process where a neutral atom has one (or more) electrons removed from their orbitals around the 
nucleus by some form of excitation, resulting in a positively charged ion, and a free negatively charged electron. The three primary mechanisms by which this occurs are photoionization, field ionization, and impact ionization. These reactions are described by the equations shown in 4 , depicting the first, second, and third ionization of an arbitrary atom, X.

$$
\begin{gathered}
\mathrm{X} \longrightarrow \mathrm{X}^{+}+\mathrm{e}^{-} \\
\mathrm{X}^{+} \longrightarrow \mathrm{X}^{2+}+\mathrm{e}^{-} \\
\mathrm{X}^{2+} \longrightarrow \mathrm{X}^{3+}+\mathrm{e}^{-}
\end{gathered}
$$

In photoionization, the electron is excited by a photon of sufficient energy to overcome the ionization potential. This photon energy can be calculated using Equation 5.

$$
E=\frac{h c}{\lambda}
$$

Where $h$ is the Planck constant $\left(6.626070 \times 10^{-34} \mathrm{~m}^{2} \mathrm{~kg} \mathrm{~s}^{-1}\right), c$ is the speed of light $\left(2.997924 \times 10^{8} \mathrm{~m} \mathrm{~s}^{-1}\right), \lambda$ is the wavelength $(\mathrm{m})$, and $E$ is the photon energy $(\mathrm{J})$ which can then be converted teV o identify the required photon wavelength for ionization to occur.

Field ionization occurs when the electric field is greater than the ionization potential of an atom. This is demonstrated using the example of the hydrogen molecule, where the ionization energy is known to be $15.43 \mathrm{eV}$. [9] The ionization field can then be determined as shown in Equations 6. [10]

$$
\begin{aligned}
& \mathcal{E} \geq \frac{15.43 \mathrm{~V}}{a_{0}} \\
& \mathcal{E} \geq 2.916 \times 10^{11} \mathrm{~V} \mathrm{~m}^{-1}
\end{aligned}
$$

Where $a_{0}$ is the Bohr radius $\left(5.291772 \times 10^{-11} \mathrm{~m}\right)$, and $\mathcal{E}$ is the minimum electric field $\left(\mathrm{V} \mathrm{m}^{-1}\right)$ capable of ionizing an atom. Conversely, impact ionization is the result of a more direct collision of electrons, where the kinetic energy is imparted to the 
bound electron by a more energetic free one. Figure 5 shows the impact ionizations cross section for $\mathrm{H}_{2}, \mathrm{H}_{2}^{+}$, and $\mathrm{H}$ as a function of impinging electron energy. These

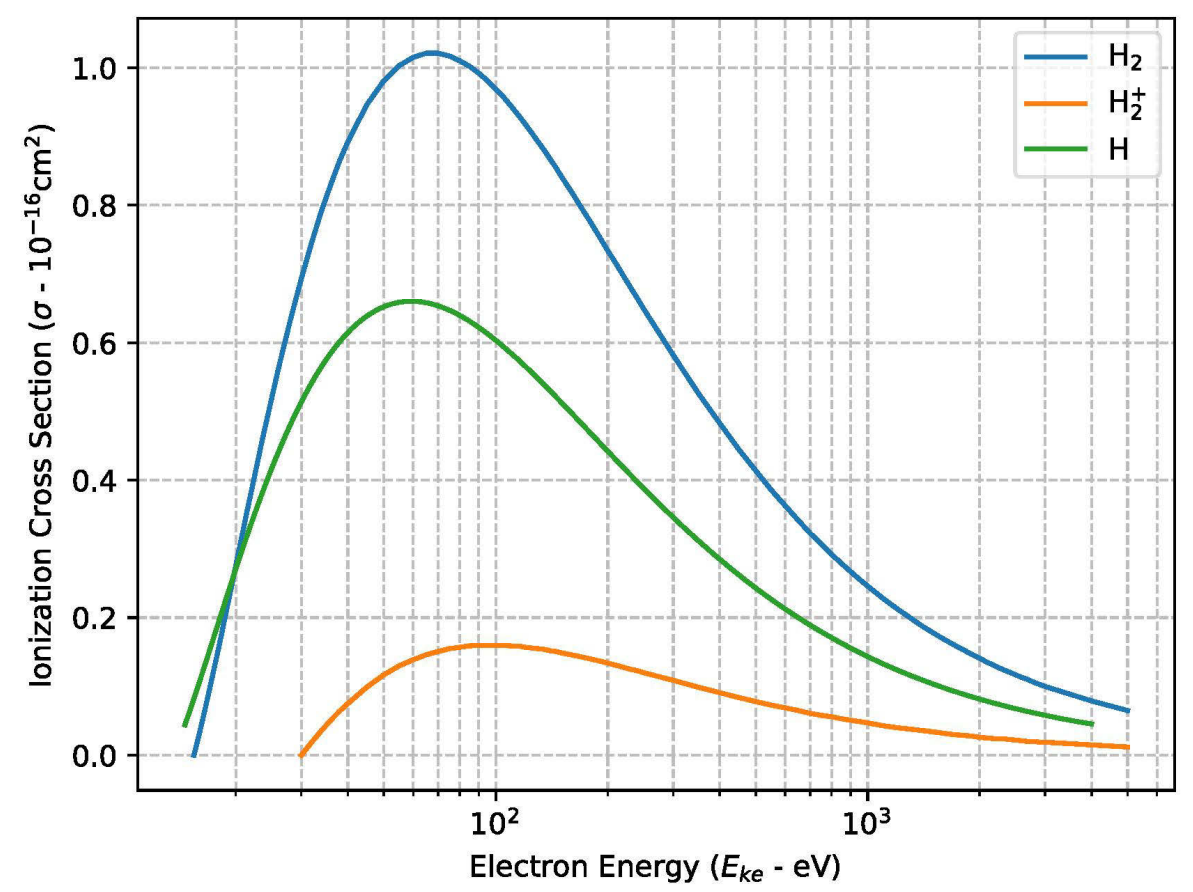

Figure 5: Impact ionization cross section for $\mathrm{H}_{2}, \mathrm{H}_{2}^{+}$, and $\mathrm{H}$

values are generated using Equations 7 for $\mathrm{H}_{2}$ and $\mathrm{H}$ [11], and Equation 8 for $\mathrm{H}_{2}^{+}$. [12]

$$
\begin{gathered}
\sigma_{B E B}=\frac{4 \pi a_{0}{ }^{2} N_{o e} R_{y}{ }^{2}}{B_{e}} \frac{1}{E_{k e}+E_{o}+B_{e}}\left[\left(\frac{E_{k e}{ }^{2}-B_{e}{ }^{2}}{2 E_{k e}^{2}}-\frac{B_{e}}{E_{k e}+B_{e}}\right) \ln \frac{E_{k e}}{B_{e}}+1-\frac{B_{e}}{E_{k e}}\right] \\
\sigma_{B E Q}= \\
\frac{8 \pi a_{0}{ }^{2} N_{o e} R_{y}{ }^{2}}{B_{e}} \frac{1}{2 E_{k e}+E_{o}+B_{e}}\left[\frac{B_{e} M_{i o n}^{2}}{N_{o e} R_{y}} \ln \frac{E_{k e}}{B_{e}}\left(1-\frac{B_{e}{ }^{2}}{E_{k e}^{2}}\right)+\right. \\
\left.2\left(1+\frac{B_{e} M_{i o n}^{2}}{N_{o e} R_{y}}\right)\left(1-\frac{B_{e}}{E_{k e}}-\frac{B_{e}}{E_{k e}+B_{e}} \ln \frac{E_{k e}}{B_{e}}\right)\right]
\end{gathered}
$$

Where $N_{o e}$ is the electron occupation number (unitless), $R_{y}$ is the Rydberg energy $(13.605693 \mathrm{eV}), B_{e}$ is the electron orbital binding energy $(\mathrm{eV}), E_{k e}$ is the electron kinetic energy $(\mathrm{eV}), E_{o}$ is the electron orbital average kinetic energy $(\mathrm{eV}), \sigma_{B E B}$ is the 
cross section for the unionized atom or diatom, and $\sigma_{B E Q}$ is for the ionized diatom. The term $M_{i o n}^{2}$ used in calculation of $\sigma_{B E Q}$ is given by Equation 9 .

$$
M_{i o n}^{2}=\int_{B_{e}}^{\infty} \frac{R_{y}}{E_{o}} \frac{d f}{d E_{o}} d E_{o}
$$

Where $\frac{d f}{d E_{o}}$ is the continuum oscillator strength, a measure of the probability of absorption of photon energy by the electron.

The ionization cross section can be useful in designing ion sources, but due to complex interactions electrons undergo between impacts, controlling for energy is not only difficult, but poorly understood and so not easily managed.

\subsubsection{Plasma Basics}

Typically, plasmas find two primary uses in fusion research, as a source for ions, or as the fusion medium itself. Plasmas, frequently referred to as the "fourth state of matter" are superheated gasses where the temperature is sufficient to ionize the constituent atoms.

A consequence of their ionized state, the separated electrons and ions move semiindependent of each other, making plasmas uniquely susceptible to control and manipulation by both electric fields, and magnetic fields. This, however, means that the interactions of the ions and electrons within the plasma is very complex as the charged particles attract and repulse one another. Despite these complex self-interactions, plasmas can often be modelled as a collective and treated as collisionless.

Due to the presence of both the ionized particle and the electrons torn from their original companions, the attraction and repulsion each experience results in the bulk of the plasma being treated as quasi-neutral. [13]. The distance over which quasineutrality can be violated while still maintaining a plasma, or the distance over which 
any one charge's influence will be screened out is known as the Debye length. [14]

$$
\lambda_{D}=\sqrt{\frac{T_{e} k \varepsilon_{0}}{n_{e}}}
$$

Where $n_{e}$ is the number density of electrons $\left(\mathrm{m}^{-3}\right), T_{e}$ is the electron temperature $(\mathrm{K}), k$ is the Boltzmann constant $\left(1.380648 \times 10^{-23} \mathrm{~J} \mathrm{~K}^{-1}\right), \varepsilon_{0}$ is the permittivity of free space $\left(8.854187 \times 10^{-12} \mathrm{~F} \mathrm{~m}^{-1}\right)$, and $\lambda_{D}$ is the Debye length $(\mathrm{m})$. It should be noted that due to the atomic nature of plasmas it is typical to discuss their thermal energy in units of electronvolts $(\mathrm{eV})$.

In laboratory settings, however, plasmas must be contained and the quasi-neutrality treatment breaks down at the interfaces with the plasma container walls, and extraction apertures. At the walls, a positive potential relative to the plasma bulk will form. This is a result of the electrons' significantly higher mobility than the ion species in the plasma meaning that for a wall electrode maintained at a constant potential, more electrons are lost to the walls than ions neutralized. [15] This region of loss of quasi-neutrality is known as the plasma sheath and has as a condition the equality shown in 11.

$$
\nu \geq \nu_{B}=\sqrt{\frac{k T_{e}}{M}}
$$

This equality is known as the Bohm sheath criterion, where $\nu_{B}$ is known as the Bohm velocity, $\nu$ is the particle velocity $\left(\mathrm{ms}^{-1}\right), T_{e}$ is the electron temperature $(\mathrm{K})$, and $M$ is the mass of ion $(\mathrm{kg})$. The meaning of this is that only ions with velocity greater than the Bohm velocity are able to enter the sheath. This is a direct consequence of the space-charge equilibrium established by the ions at the plasma wall.

In addition to the plasma sheath, there is an associated presheath region between the sheath and bulk, where the quasineutrality is re-established. For a collisionless 
sheath, the potential of the presheath is given by Equation 12.

$$
\phi_{P}=\frac{k T_{e}}{2 e}
$$

Where $T_{e}$ is the electron temperature $(\mathrm{K})$. The equation for the potential between the edge of the presheath and the wall is given by Equation 13 .

$$
\phi_{W}=\frac{-k T_{e}}{2 e} \ln \left(\frac{M}{2 \pi m_{e}}\right)
$$

Where $T_{e}$ is the electron temperature $(\mathrm{K}), M$ is the mass of ion $(\mathrm{kg})$, and $m_{e}$ is the mass of electron $\left(9.109383 \times 10^{-31} \mathrm{~kg}\right)$. These can then be combined to find the overall potential between the plasma bulk, and the containment wall, called the plasma potential $\left(\phi_{P W}\right)$ given in Equation 14 .

$$
\phi_{P W}=\phi_{P}-\phi_{W}=\frac{k T_{e}}{2 e}\left(1+\ln \left(\frac{M}{2 \pi m_{e}}\right)\right)
$$

\subsubsection{Plasma Sources}

There exist a variety of plasma sources that can be classified by their principal plasma formation mechanism. These include Direct Current (DC) sources, and RF sources, which can then be subdivided by important features. [16] [17] [18]

- DC source

- Cold cathode source

- Electron bombardment source

- Duoplasmatron source

- Capillary arc source

- RF Sources 
- Electron Cyclotron Resonance (ECR) ion source

- Microwave source

- Inductively coupled RF sources

- Capacitively coupled RF sources

This list, however, should not be considered exhaustive, as new forms and combinations are continuously being developed. Despite this, it does give a good overview major types of plasma based ion sources. In considering which source to develop for initial testing, and important to consider requirements of specialty materials, cooling, power supplies, and space requirements.

$\mathrm{RF}$ sources are notable for requiring sufficiently high power RF supplies, as well as specialized ceramic or glass plasma containment vessels. These requirements present an added complexity that makes maintenance costlier and more difficult to do inhouse. Similarly, of the DC sources, capillary arc source, and duoplasmatrons require complex geometries, and active cooling respectively to operate, both of which are undesirable when trying to minimize space needed by the source, and the complexity of maintenance. Owing to its simplicity of design, ease of manufacture, and operation, the more desirable choice for application in the development of a compact, self contained experimental apparatus, are the DC cold cathode sources known as Penning Ion Generator (PIG)s. An interesting additional plasma concept is the potential for self-contained plasmas that, by way of their own fields, maintain their shape for some time (typically confinement on the order of microseconds). Three such configurations that have been investigated and produced in laboratory settings are Compact Toroid (CT), Field-Reversed Configuration (FRC), and spheromaks. 


\subsubsection{Penning Ion Source}

The basic design that is being used in this work is the PIG, a cold cathode DC ion source. In a PIG electrons are emitted from the cathodes by Field Emission (FE), and accelerated towards the anode. By applying a magnetic field, the electrons spiral and subsequently oscillate between the two cathodes, never quite reaching the anode. The basic form of the PIG source is shown in Figure 6, where the blue cylinder is the outer containment wall and both the top and bottom cathode with an extraction hole in the top cathode. The inner tube is the anode that will surround the formed plasma. The magnetic field that spirals the electrons is denoted by $B$. In this PIG design, the

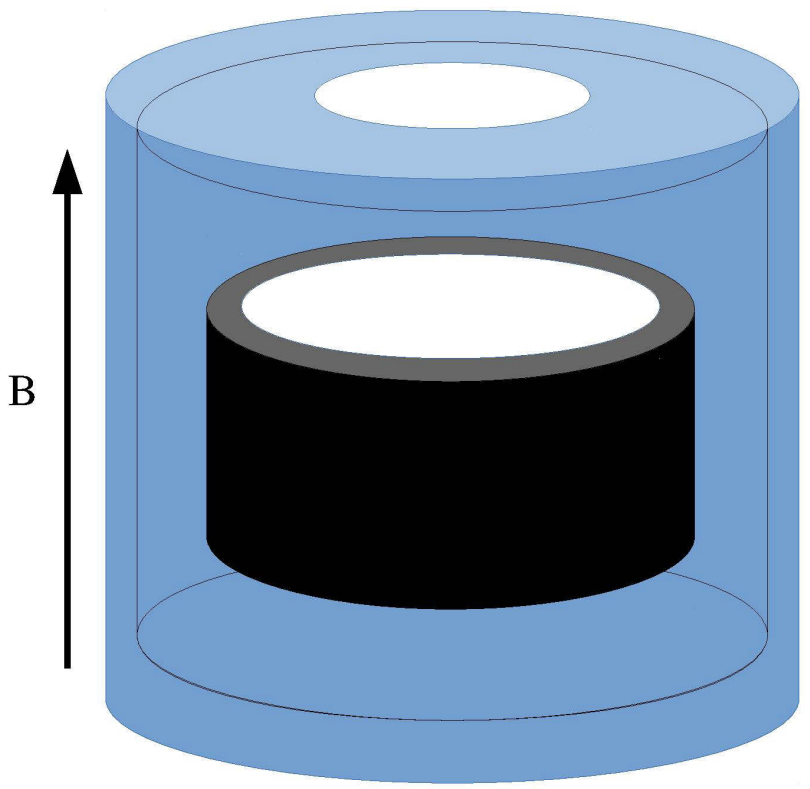

Figure 6: Sketch of PIG basic design

magnetic field is applied by a single permanent magnet locate concentrically around the anode ring; and operates on the following principle.

1. Electrons are emitted by FE and travel towards the anode

2. The magnetic field causes the electron's trajectory to spiral 
3. The cathode at the opposite end reflects the electrons back down, resulting in an oscillatory motion

4. The trapped electrons continually strike neutral atoms, ionize them, and accelerate again before striking another

5. A plasma is formed and maintained within the anode tube

As the gas pressure in the cavity will define the path length of the electron, a typical range for operation is between $\approx 1$ Torr and $1 \times 10^{-3}$ Torr. Another important parameter is the magnetic field strength of which $0.1 \mathrm{~T}$ is strong enough to increase the path lengths of the electrons sufficiently. [19]

The advantage of a cold cathode source like the PIG, is that it is compact, and operates at low gas pressures, and so can be housed within the vacuum chamber itself, simplifying the overall system design. In addition to this, it is simple to construct, and as a result, easily maintained. It has drawbacks, however, including greater risk of metal impurities in the formed ion beam, as well as lower efficiency compared to other DC sources, or RF sources. [18]

\subsection{Ion Beams}

\subsubsection{Extraction From Plasmas}

Given the complexity of interactions that occur within plasmas, extracting useable ion beams presents a unique challenge. In order to have the majority of extracted ions striking the intended target and not the chamber walls, it is important that the initial extracted beam be uniform and cylindrical. A key consideration in achieving a uniform beam is the plasma meniscus at the point of extraction.

As previously mentioned, space-charge plays an important role in a plasma's behaviour. Particularly at the extraction orifice of a plasma ion source, the ions form a region known as the plasma meniscus where the space-charge build-up will affect 
both the maximum extractable current, and the shape of the extracted beam. The space-charge limited current density is given by the Child-Langmuir Equation 15. [19]

$$
J=\frac{4}{9} \varepsilon_{0} \sqrt{\frac{2 e}{M}} \frac{V^{3 / 2}}{L^{2}}
$$

Where $M$ is the mass of ion $(\mathrm{kg})$ forming the plasma, $V$ is the applied voltage $(\mathrm{V})$ between the plasma electrode and extraction electrode, and $L$ is the electrode separation $(\mathrm{m})$. When extracting an electron beam, the solution for an optimal cylindrical beam is given by the Pierce angle, which has a value of $67.5^{\circ}$ relative to the direction of travel of the electron beam as shown in Figure 7. [20] This, however, does not

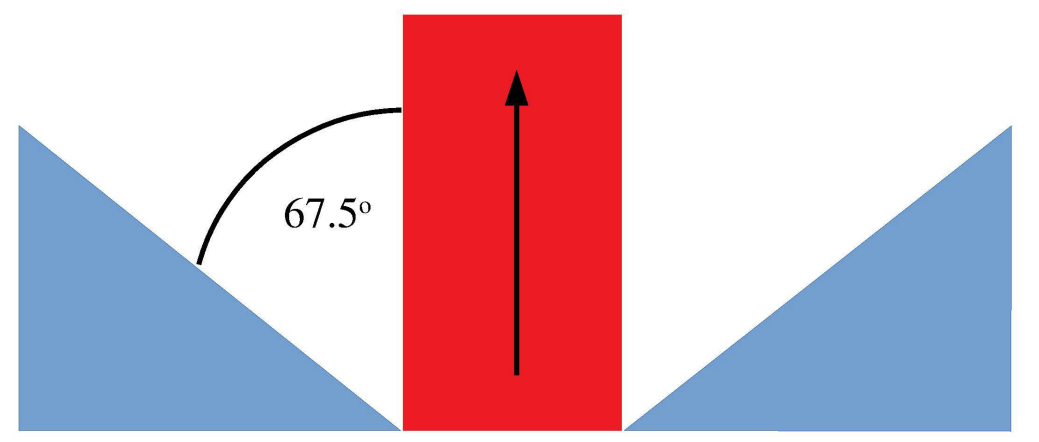

Figure 7: Diagram showing the Pierce angle on the extraction electrode (blue) as applied to an extracted electron beam (red) with direction of travel indicated by the arrow

apply to ion extraction systems due to the plasma meniscus warping the shape of the equipotential lines at the extraction region.

The optimal shape for extracting a uniform cylindrical ion beam is instead given by a hyperbola, with a radius of curvature dependent on the Debye length of the plasma. [21] This can be simplified and approximated by a circle, for ease of manufacturing. The radius of the circle is found using the Equation 16, which in turn calls on Equations 17 through 20, where $\lambda_{D}$ is the Debye length (m), $M$ is the mass of ion $(\mathrm{kg}), E_{k e}$ is the electron kinetic energy $(\mathrm{eV})$, and $E_{k i}$ is the ion kinetic energy $(\mathrm{eV})$. For many plasmas, this ratio $\beta$ (Equation 20) can be taken as 1, as the ions 
and electrons have roughly equivalent kinetic energy. [21]

$$
\begin{gathered}
\frac{R}{\lambda_{D}}=\frac{\eta_{w}^{\prime}}{\eta_{w}^{\prime \prime}} \\
\eta_{w}^{\prime}=2 \sqrt{\frac{\sqrt{1+\beta \eta_{w}}-1}{\beta}+\frac{\exp \left[-\eta_{w}\right]-1}{2}} \\
\eta_{w}^{\prime \prime}=\frac{1}{\sqrt{1+\beta \eta_{w}}}-\frac{1}{\exp \left[\eta_{w}\right]} \\
\eta_{w}=\frac{1}{2} \ln \left\{\frac{\beta M}{\pi m_{e}}\right\} \\
\beta=\frac{E_{k e}}{E_{k i}}
\end{gathered}
$$

\subsubsection{Manipulation and Control of Ions and Ion Beams}

In order to be useful in fusion experiments once extracted, it is necessary to accelerate the ions to sufficient energies that the cross section is as high as possible to increase the probability of a fusion event. Acceleration can be accomplished either by a simple accelerating gap, where a large voltage is applied between the source and the target, or by way of RF cavities (typically superconducting in modern incarnations). As before with the ion source, the RF cavities were not chosen due to the large volumes required to operate them, and more complex equipment required. An accelerating gap was thus preferred choice for initial tests. An electronvolt, is defined as the kinetic energy of an electron accelerated across a potential of $1 \mathrm{~V}$. As such, the energy given

to a fully ionized particle travelling across an accelerating gap $V_{g a p}=V_{2}-V_{1}$ is given by Equation 21 .

$$
E_{k}=Z e\left(V_{2}-V_{1}\right)
$$


Where $Z$ is the number of protons in nucleus (atomic mass number - unitless), $e$ is the elementary charge $\left(1.602176 \times 10^{-19} \mathrm{C}\right)$, and $V_{1}$ and $V_{2}$ are the accelerating electrode potentials. With an ion beam extracted, all charged species will be of the same charge, and thus self-repelling. Because of this, it is necessary to steer or otherwise manipulate the beam to keep these space-charge effects from blowing it apart, and subsequently reducing the current at the target.

Ion beam steering, often referred to as ion optics, are a set of methods for controlling the size, shape, direction and even components of an ion beam. Manipulation can be accomplished either by electric fields, magnetic fields, or a combination of the two.

Electrostatic manipulation of beams typically involves plates, tubes, or apertures onto which a potential is applied. These surfaces then attract or repel the ions depending on the charge. With magnetic manipulation, on the other hand, the ions experience a force known as the Lorentz force. This causes particles to spiral in an arc with radius given by the Equation 22 .

$$
R=\frac{m \nu}{q B}
$$

Where $m$ is the particle mass $(\mathrm{kg}), \nu$ is the particle velocity $\left(\mathrm{m} \mathrm{s}^{-1}\right), q$ is the charge $(\mathrm{C})$, and $B$ is the magnetic field $(\mathrm{T})$. If the particles have been accelerated by a potential of $V$, the radius of curvature for the path can, for ease of use, be rewritten as in Equation 23. [22]

$$
R=\frac{1}{B} \sqrt{\frac{2 m V}{q}}
$$

Where the accelerating potential $V$ is used in place of the particle's velocity. This behaviour of charged particles in magnetic fields is exploited in many ways, including in the plasma sources themselves as was described previously. Magnetic fields also typically have a much stronger effect on the particles than electrostatics, but can 
be selected against for size considerations and simplicity of electrostatic optics. As suggested by the name, ion optics can in many scenarios be treated similarly to geometric optics in defining the beam path taken by test particles. In simple cases, this allows for matrix calculations of a particle's trajectory. [22] Where a particle's distance from the central axis is given by $r$ and the slope of its deflection is given by $r^{\prime}$, its trajectory in a uniform field is described by the transfer matrix given in Equation 24.

$$
\left(\begin{array}{l}
r \\
r^{\prime}
\end{array}\right)_{2}=\left(\begin{array}{cc}
1 & \frac{2 L}{\sqrt{\frac{V_{1}}{V_{2}}+1}} \\
0 & \sqrt{\frac{V_{1}}{V_{2}}}
\end{array}\right)\left(\begin{array}{l}
r \\
r^{\prime}
\end{array}\right)_{1}
$$

Where $L$ is the electrode separation (m), and $V_{1}$ and $V_{2}$ are the accelerating electrode potentials. An aperture with a uniform field on one side and a different uniform field on the other is then given by Equation 25 .

$$
\left(\begin{array}{l}
r \\
r^{\prime}
\end{array}\right)_{2}=\left(\begin{array}{cc}
1 & 0 \\
\frac{\mathcal{E}_{1}-\mathcal{E}_{2}}{4 V_{a}} & 1
\end{array}\right)\left(\begin{array}{l}
r \\
r^{\prime}
\end{array}\right)_{1}
$$

Where $\mathcal{E}_{1}$ and $\mathcal{E}_{2}$ are the electric field $\left(\mathrm{V} \mathrm{m}^{-1}\right)$ before and after the aperture, and $V_{a}$ is the accelerating potential. These two can then be combined to describe the effects of a series of apertures and fields on the trajectory of particles. One particularly useful example of this is the Einzel lens. An Einzel lens is a series of three apertures where $V_{1}=V_{3}$ and either $V_{1}>V_{2}$ or $V_{1}<V_{2}$. If the first gap of the Einzel lens is accelerating the incoming particles, the lens is said to be "accelerating", otherwise, it is dubbed "decelerating".

These terms accelerating and decelerating are in actuality misnomers, as the Einzel lens is designed specifically not to change a beam's energy, but rather refocus it and maintain its shape. The difference in accelerating and decelerating lenses is in its 
refractive power, and the spherical aberration the beam may experience. Accelerating lenses have much lower refractive power, and so often, much higher potentials are required in order to achieve the same effect as a decelerating lens. The accelerating lenses, however, are preferable due to the lower spherical aberration, which can greatly affect the beam quality. [23] The transfer matrix for an Einzel lens can be found by performing matrix multiplication of an aperture as given previously, followed by a uniform field, then a second aperture, a second uniform field, and finally a third aperture. This equation, however, assumes a uniform field between the elements, and thus doesn't have good agreement with simulation or real systems when the diameter of the aperture approaches the separation of the elements, or if the electrodes aren't flat and perpendicular to the axis of particle motion. The transfer matrix for the Einzel lens is given by Equation 26.

$$
\begin{aligned}
\left(\begin{array}{l}
r \\
r^{\prime}
\end{array}\right)_{2}= & \left(\begin{array}{cc}
1 & 0 \\
\frac{\mathcal{E}_{1}-\mathcal{E}_{2}}{4 V_{a}} & 1
\end{array}\right)_{1}\left(\begin{array}{cc}
1 & \frac{2 L}{\sqrt{\frac{V_{1}}{V_{2}}}+1} \\
0 & \sqrt{\frac{V_{1}}{V_{2}}}
\end{array}\right)_{12}\left(\begin{array}{cc}
1 & 0 \\
\frac{\mathcal{E}_{1}-\mathcal{E}_{2}}{4 V_{a}} & 1
\end{array}\right)_{2} \\
& \left(\begin{array}{ll}
1 & \frac{2 L}{\sqrt{\frac{V_{1}}{V_{2}}}+1} \\
0 & \sqrt{\frac{V_{1}}{V_{2}}}
\end{array}\right)_{23}\left(\begin{array}{cc}
1 & 0 \\
\frac{\mathcal{E}_{1}-\mathcal{E}_{2}}{4 V_{a}} & 1
\end{array}\right)_{3}\left(\begin{array}{l}
r \\
r^{\prime}
\end{array}\right)_{1}
\end{aligned}
$$

Where the subscripts denote the aperture as viewed along the particles trajectory, and the fields between the apertures. An important tool in characterizing beams are emittance plots. Emittance plots are 2D plots that capture phase space information. Phase space has 6 dimensions $(x, y, z)$ and $\left(p_{x}, p_{y}, p_{z}\right)$ representing spatial positioning and momentum respectively.

$$
x^{\prime}=\frac{p_{x}}{p_{z}}
$$


By taking a particle's $x$ position, and plotting it against $x^{\prime}$, defined in Equation 27, it is possible to paint a picture of the particles overall behaviour. This has the added benefit of being able easily show when a beam's phase space profile follows a Gaussian distribution, as a 2D Gaussian has an ellipse of constant sigma which allows for easy identification of the behaviour of the majority of particles. [15] Figure 8 demonstrates the ellipses for three cases, where the beam is converging (Figure 8a), where the beam is focused (Figure 8b), and where the beam is diverging (Figure 8c). [24] As such,

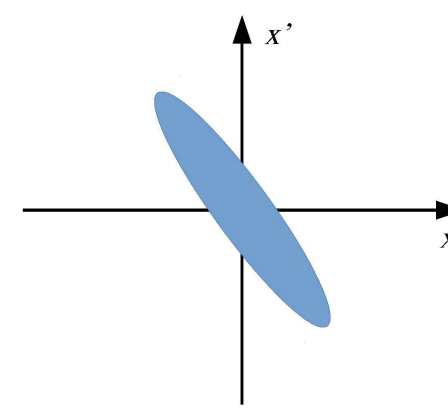

(a) Converging

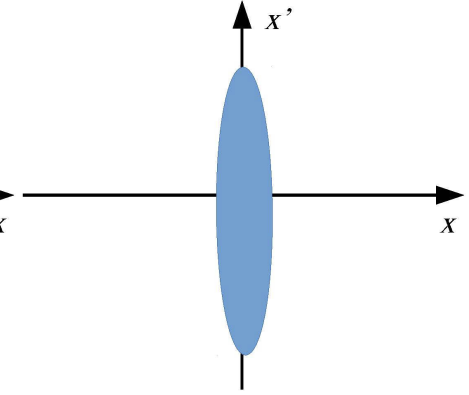

(b) Focused

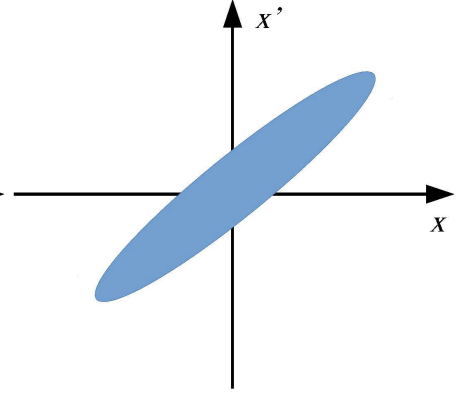

(c) Diverging

Figure 8: Sketches of emittance ellipses and interpretation of particle behaviour

the degree to which the beam is divergent or convergent can be interpreted from the slope of the ellipse. The greater the slope, the more severely divergent or convergent, and conversely, a shallow slope would indicate a gradual divergence or convergence.

\subsection{Fusion}

\subsubsection{Calculating Energy Release}

Similarly to chemical reactions, nuclear reactions can be described as endothermic (requires energy input to occur) or exothermic (releases energy and occurs spontaneously). Which a reaction is, is determined by the difference in binding energy of the reactants and products of the process. Nuclear binding energy can be calculated using the semi-empirical formula seen in Equation 28, known as the semi-empirical 
mass formula, or the Weizsäcker mass formula. [25]

$$
E_{B}(Z, N)=\alpha_{1} A-\alpha_{2} A^{2 / 3}-\alpha_{3} \frac{Z(Z-1)}{A^{1 / 3}}-\alpha_{4} \frac{(N-Z)^{2}}{A}+\Delta
$$

Where $A$ is the number of nucleons (protons and neutrons) in the nuclei, $Z$ the number of protons, and $N$ the number of neutrons. The coefficients $\alpha_{1-4}$ and $\Delta$ are factors determined empirically, but $\Delta$ known as the pairing energy parameter, has an additional condition on it, as described in 29.

$$
\Delta= \begin{cases}\delta, & \text { for } A \text { and } N \text { both even } \\ 0, & \text { for } A+N \text { odd } \\ -\delta, & \text { for } A \text { and } N \text { both odd }\end{cases}
$$

As with the other terms of the Weizsäcker mass formula, $\Delta$ is empirically derived. Typical values for all of the coefficients can be found in Table 4. [25] As its name

Table 4: Commonly used coefficients for binding energy calculation

\begin{tabular}{c|c|c|c|c}
$\alpha_{1}$ & $\alpha_{2}$ & $\alpha_{3}$ & $\alpha_{4}$ & $\delta$ \\
\hline $16 \mathrm{MeV}$ & $17 \mathrm{MeV}$ & $0.6 \mathrm{MeV}$ & $25 \mathrm{MeV}$ & $\frac{25}{A} \mathrm{MeV}$
\end{tabular}

indicates, this formula is a combination of theory and experimental results, with the coefficients being defined by fits to data. As such, the values or the coefficients change depending on the data set, and frequently don't adequately represent the binding energy of lower mass nuclei. The formula was born in an effort to create a formula that would give the binding energy from only the numbers of nucleons in the nuclei.

Using the standard atomic mass unit (also known as a Dalton Da) multiplied by the number of nucleons was insufficient because the Dalton, defined as one twelfth of a ${ }^{12} \mathrm{C}$ mass does not account for the mass energy equivalence, defined by the wellknown equation $E=m c^{2}$. This gave rise to the term of mass defect, to refer to 
the lower than expected mass of the lighter nuclei, a result of the strong binding energy. This mass defect is well measured experimentally and, in calculating energy released from a nuclear reaction, it is frequently more practical to use the difference in total mass defect, as a shortcut in determining energy release or absorbed. [26] All calculations and plots contained herein will therefore be based only on data from the Nation Nuclear Data Center.

Figure 9 shows a simple visualization of the fusion process for deuterium-deuterium $\left({ }^{2} \mathrm{H}_{-}{ }^{2} \mathrm{H}\right)$ fusion, a common reaction investigated in fusion power research. This visualization also demonstrates the two possible product pathways of the reaction, both with equal probability of occurring. Using the aforementioned mass defect, the energy

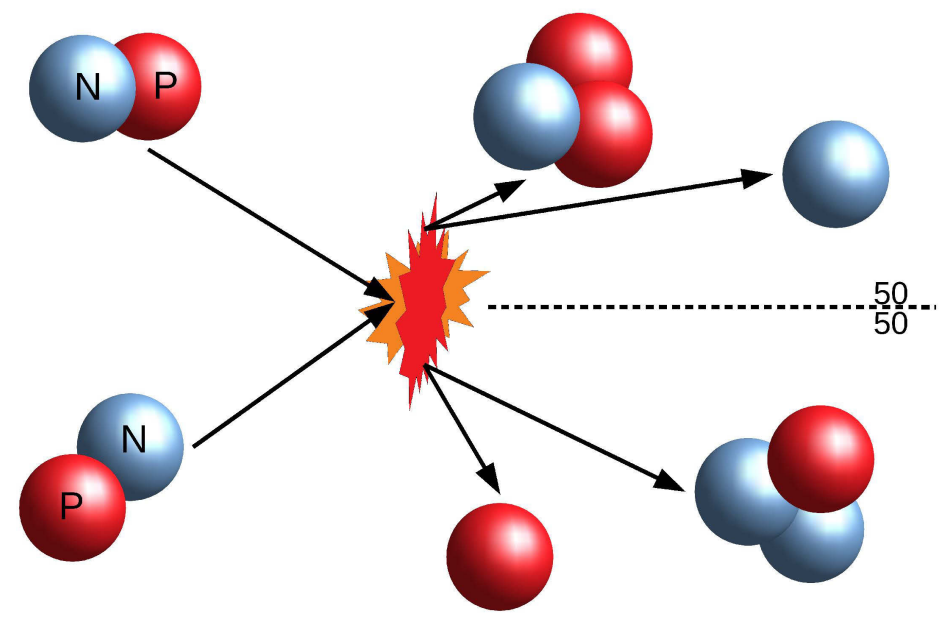

Figure 9: A visualization of the possible fusion pathways for ${ }_{1}^{2} \mathrm{H}-{ }_{1}^{2} \mathrm{H}$ fusion.

that a reaction will release (the change in binding energy $\Delta E_{B}$ ) can be calculated, as shown in Equation 30. This calculation is done for the ${ }^{3} \mathrm{He}-\mathrm{n}^{0}$ reaction pathway of 
the aforementioned ${ }^{2} \mathrm{H}-{ }^{2} \mathrm{H}$ reaction.

$$
\begin{aligned}
\Delta E_{B} & \equiv \sum E_{D}(\text { Reactants })-\sum E_{D}(\text { Products }) \\
\Delta E_{B} & =\left(E_{D}\left({ }^{2} \mathrm{H}\right)+E_{D}\left({ }^{2} \mathrm{H}\right)\right)-\left(E_{D}\left({ }^{3} \mathrm{He}\right)+E_{D}\left(\mathrm{n}^{0}\right)\right) \\
\Delta E_{B} & =(13.135 \mathrm{MeV}+13.135 \mathrm{MeV})-(14.931 \mathrm{MeV}+8.071 \mathrm{MeV}) \\
\Delta E_{B} & =3.27 \mathrm{MeV}
\end{aligned}
$$

Where $E_{D}$ is the mass defect $(\mathrm{eV})$ of the component. When investigating many fusion (or even fission fuels) the most helpful visualization of energy release for nuclear reactions isn't just the binding energy/mass defect, as they typically follow a linear pattern for increasing atomic mass. It is often more informative to look instead at the binding energy per nucleon. This is the curve shown in Figure 10. Figure 10 shows

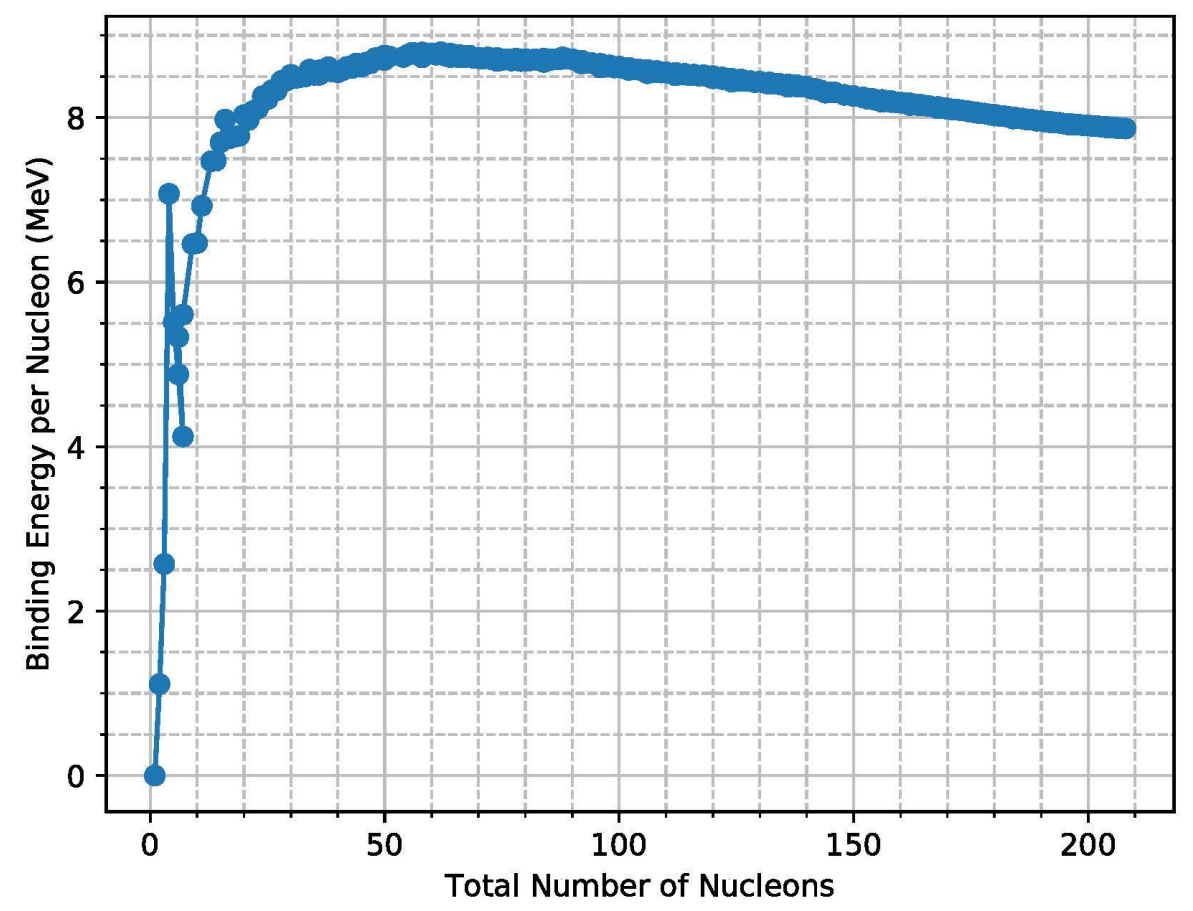

Figure 10: Binding energy per nucleon of stable nucleons

that up to about 60 nucleons, the binding energy per nucleon is increasing, whereas beyond that the binding energy per nucleon decreases albeit much more gradually. 
This is the well-known pathway for nucleosynthesis of elements up to ${ }^{56} \mathrm{Fe}$ in stars, where fusion produces energy until the proportion of iron is too great. Similarly, it demonstrates the source of current nuclear fission power, where splitting heavier elements results in lighter ones that move back towards iron and thus release energy. This chart demonstrates very clearly the appeal of fusion as a power source. The change in binding energy per nucleon is significantly greater moving up towards iron than it is moving back from the heavier elements, giving it much greater energy release for its mass.

\subsubsection{The Fusion Process}

Fusion is a process where two fundamental forces are pitted against one another, the strong nuclear force against the electromagnetic force. In order for two nuclei to fuse, they must get close enough that one may tunnel through the Coulombic barrier of the other, to where the extremely powerful attractive force of the strong nuclear force overpowers the repulsive Coulombic force. As shown in Table 5, the

Table 5: Fundamental forces and their basic properties

\begin{tabular}{c|c|c|c} 
Interaction & $\begin{array}{c}\text { Field } \\
\text { Quantum }\end{array}$ & $\begin{array}{c}\text { Range } \\
(\mathrm{m})\end{array}$ & $\begin{array}{c}\text { Relative } \\
\text { Strength }\end{array}$ \\
\hline Strong & Gluon & $10^{-15}$ & 1 \\
\hline Weak & $W^{ \pm}, Z^{0}$ & $10^{-18}$ & $10^{-5}$ \\
\hline Electromagnetic & Photon & $\infty$ & $\alpha=\frac{1}{137}$ \\
\hline Gravity & Graviton & $\infty$ & $10^{-38}$
\end{tabular}

electromagnetic force is $1 / 137^{\text {th }}$ the strength of the strong nuclear force, but the strong nuclear force has a range only on the order of a femtometer. The consequence of this range and strength imbalance is the potential of two approaching nuclei having the shape shown in Figure 11, where the Coulombic barrier results in a strongly repulsive field that is then overpowered by the much more attractive strong nuclear force when the nuclei get within a few femtometers of one another. In real fusion 


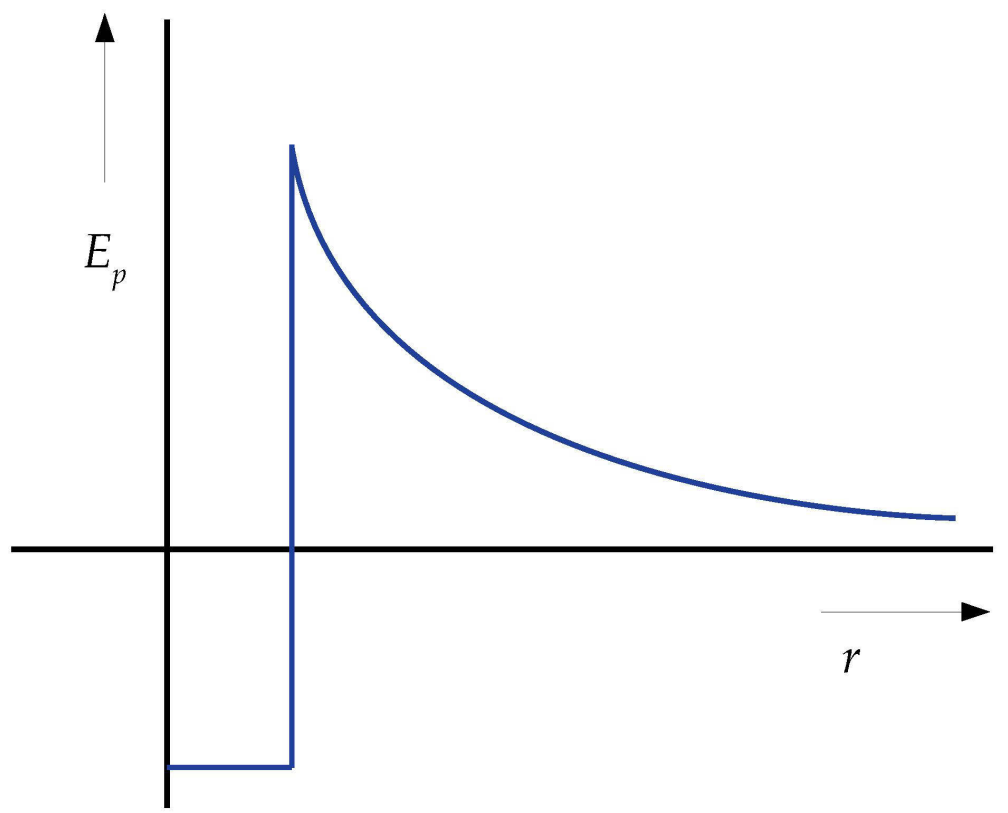

Figure 11: Sketch of the approximate potential well of a nucleus

conditions this is investigated in a more probabilistic manner, because of quantum effects like quantum tunnelling, rather than a binary distinction between sufficient or insufficient energy to overcome the barrier, there exists a continuum of energies along which fusion has a sufficiently high probability of occurring. When calculating fusion rates, interaction cross sections are used as a measure of the probability of a certain event happening. Elastic scattering or inelastic scattering (fusion) have different cross sections that are energy dependent, and typically experimentally determined. The Nuclear Energy Agency (NEA) maintains a compendium of cross section data known as Java-based Nuclear Data Information System (JANIS) from which fusion cross sections for a variety of reactions can be found. [27] In using this database, it is important to understand the notation typically used in discussing fusion reactions.

$$
{ }^{2} \mathrm{H}\left({ }^{2} \mathrm{H},{ }^{3} \mathrm{He}+\mathrm{n}^{0}\right)
$$

Shown above in 31, is a common way of denoting fusion reactions. It shows first the target, then the impinging particle, and finally the products. If there is just one it 
is written inside the brackets, but if there are more, they are continued outside the brackets. Table 6 shows a number of important nuclear reactions considered when

Table 6: Important Reaction in Nuclear Fusion

\begin{tabular}{|c|c|c|}
\hline Reactants & $\begin{array}{c}\text { Products with Energy Distribution } \\
\text { and Pathway Probability }\end{array}$ & Shorthand \\
\hline${ }^{1} \mathrm{H}+{ }^{6} \mathrm{Li}$ & $\underset{4.00 \mathrm{MeV}}{ }{ }_{1.7 \mathrm{MeV}}^{4}+{ }^{{ }^{3} \mathrm{He}}$ & ${ }^{6} \mathrm{Li}\left({ }^{1} \mathrm{H},{ }^{4} \mathrm{He}+{ }^{3} \mathrm{He}\right)$ \\
\hline${ }^{1} \mathrm{H}+{ }^{7} \mathrm{Li}$ & $17.2 \mathrm{MeV} 22{ }^{4} \mathrm{He}$ & ${ }^{7} \mathrm{Li}\left({ }^{1} \mathrm{H},{ }^{4} \mathrm{He}+{ }^{4} \mathrm{He}\right)$ \\
\hline${ }^{1} \mathrm{H}+{ }^{11} \mathrm{~B}$ & $8.68 \mathrm{MeV} \rightarrow 3{ }_{2.9 \mathrm{MeV}}^{4} \mathrm{He}$ & ${ }^{11} \mathrm{~B}\left({ }^{1} \mathrm{H},{ }^{4} \mathrm{He}+{ }^{4} \mathrm{He}\right)$ \\
\hline${ }^{2} \mathrm{H}+{ }^{2} \mathrm{H}$ & $\underset{4.03 \mathrm{MeV}}{50 \%} \underset{1.01 \mathrm{MeV}}{{ }^{3} \mathrm{H}}+{ }_{3.02 \mathrm{MeV}}^{1} \mathrm{H}$ & ${ }^{2} \mathrm{H}\left({ }^{2} \mathrm{H},{ }^{3} \mathrm{H}+{ }^{1} \mathrm{H}\right)$ \\
\hline & $\underset{3.27 \mathrm{MeV}}{\stackrel{5}{3} \mathrm{He}}+\underset{0.82 \mathrm{MeV}}{\mathrm{n}^{0}}$ & ${ }^{2} \mathrm{H}\left({ }^{2} \mathrm{H},{ }^{3} \mathrm{He}\right) \mathrm{n}^{0}$ \\
\hline${ }^{2} \mathrm{H}+{ }^{3} \mathrm{H}$ & $\overrightarrow{17.6 \mathrm{MeV}}{ }_{3.5 \mathrm{MeV}}^{{ }^{3} \mathrm{He}}+\underset{14.1 \mathrm{MeV}}{\mathrm{n}^{0}}$ & ${ }^{2} \mathrm{H}\left({ }^{3} \mathrm{H},{ }^{4} \mathrm{He}+\mathrm{n}^{0}\right)$ \\
\hline${ }^{2} \mathrm{H}+{ }^{3} \mathrm{He}$ & $\overrightarrow{18.3 \mathrm{MeV}}{ }_{3.6 \mathrm{MeV}}^{4} \mathrm{He}+{ }_{14.7 \mathrm{MeV}}^{\mathrm{I}} \mathrm{H}$ & ${ }^{2} \mathrm{H}\left({ }^{3} \mathrm{He},{ }^{4} \mathrm{He}+{ }^{1} \mathrm{H}\right)$ \\
\hline${ }^{2} \mathrm{H}+{ }^{6} \mathrm{Li}$ & 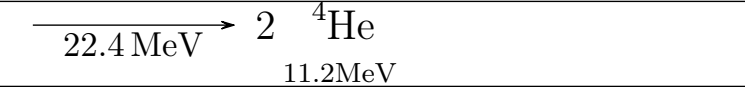 & ${ }^{2} \mathrm{H}\left({ }^{6} \mathrm{Li}, 2{ }^{4} \mathrm{He}\right)$ \\
\hline${ }^{3} \mathrm{H}+{ }^{3} \mathrm{H}$ & 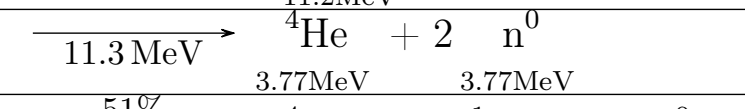 & ${ }^{3} \mathrm{H}\left({ }^{3} \mathrm{H},{ }^{4} \mathrm{He}+2 \mathrm{n}^{0}\right)$ \\
\hline${ }^{3} \mathrm{He}+{ }^{3} \mathrm{H}$ & $\underset{12.1 \mathrm{MeV}}{151 \%} \underset{4.03 \mathrm{MeV}}{{ }^{4} \mathrm{He}}+\underset{4.03 \mathrm{MeV}}{{ }^{1} \mathrm{H}}+\underset{4.03 \mathrm{MeV}}{\mathrm{n}^{0}}$ & ${ }^{3} \mathrm{He}\left({ }^{3} \mathrm{H},{ }^{4} \mathrm{He}+{ }^{1} \mathrm{H}+\mathrm{n}^{0}\right)$ \\
\hline & $\underset{\substack{6 \% \\
14.3 \mathrm{MeV}}}{{ }_{0.5 \mathrm{MeV}}^{4} \mathrm{He}}+{ }_{11.9 \mathrm{MeV}}^{1}+\underset{1.9 \mathrm{MeV}}{\mathrm{n}^{0}}$ & ${ }^{3} \mathrm{He}\left({ }^{3} \mathrm{H},{ }^{4} \mathrm{He}+{ }^{1} \mathrm{H}+\mathrm{n}^{0}\right)$ \\
\hline & 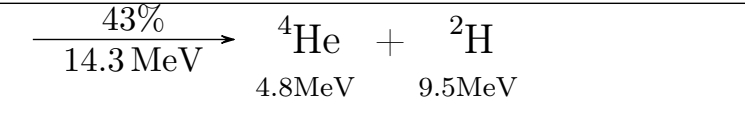 & ${ }^{\circ} \mathrm{F}$ \\
\hline
\end{tabular}

discussion fusion power sources. [28] The Table shows the total energy of the reaction underneath the reaction arrow, the energy carried away by each of the products, and where there are multiple possible reaction pathways, the probability of each path is shown.

$$
R_{r}=1-\Phi \exp (-n \sigma z)
$$

Equation 32 shows the equation used to calculate the number of reactions $\left(R_{r}\right)$ given a particle flux $\Phi$, where $n$ is the number density $\left(\mathrm{cm}^{3}\right), \sigma$ is the interaction cross section $\left(\mathrm{b} / 1 \times 10^{-24} \mathrm{~cm}^{2}\right)$, and $z$ is the penetration depth $(\mathrm{cm})$. It is tempting to think that 
the reaction rate can be increased by increasing the thickness of a material, but this quickly breaks down as energy is lost very quickly due to the many other interactions a particle will experience in a solid. A particle will typically lose on the order of $10 \mathrm{keV} \mathrm{mm}^{-1}$. [14] 


\section{State of the Art}

\subsection{Fusion Experiments}

Fusion power has been an active area of research since the 1950s, with the majority of the research funding and effort concentrated on either Inertial Confinement (IC) or Magnetic Confinement, and achieving ignition. Ignition in fusion experiments is a point where the fuel is heated more quickly by the fusion reactions than heat is lost to the environment. It is considered to be an important step to achieving a fusion power plant for many fusion techniques.

A major IC project is the National Ignition Facility (NIF) operated by the Lawrence Livermore National Laboratory. NIF uses a $500 \mathrm{TW}$ laser focused on a pellet containing a mixture of deuterium and tritium to achieve ignition. This assembly is used not just for fusion research but for maintenance and investigation of nuclear weapons, as NIF is capable of producing environments needed for the study of the conditions experienced by nuclear weapons. The majority of MC research has been focused on the development of tokamaks, a toroidally shaped magnetic containment vessel for plasmas. The objective being to achieve ignition within the plasma by heating it sufficiently, then maintaining it using the magnetic field of the torus. This is the basic principle for the major European International Tokamak Experimental Reactor (ITER) project. A similar MC project to tokamaks are stellerators. Stellerators are similar to tokamaks in their overall plasma confinement, but differ by the geometry used. Instead of simple toroidal confinement vessel, a twisting series of figure 8 magnets are used. The reason for this design variation is that in a tokamak, the magnetic fields necessarily become stronger at the inner edge of the torus, and weaker at the outer edges resulting in an asymmetric drift and subsequent loss of particles. By having solenoids with odd shapes twisting around the plasma core, the field lines followed by the particles would move out and back in towards the center cancelling out much of 
the undesirable drift. As of yet, no fusion experiment has been successful in achieving ignition or breakeven despite the decades of development and advancement in plasma research. There are, however, many privately funded research companies working to develop commercial reactors that use techniques entirely different from the typical MC or IC. Two notable groups are General Fusion (GF) and TAE Technologies.

GF in contrast to many other groups, does not seek to achieve ignition, but instead aims to generate a stable system that generates sufficient energy surplus on multiple "shots" per second. These shots operate by way of launching spheromaks from opposing sources, having them collide to form a stationary plasma core, and then collapsing a liquid lead lithium blanket onto the plasma core using hydraulic pistons. This collapsing wall compresses the plasma core and heats it sufficiently to generate fusion that then in turn expands and gives off heat to the liquid metal blanket which can be extracted through a heat exchanger. TAE Technologies similarly use opposing self contained plasmas launched at one another to achieve fusion, but has the unique approach of using the ${ }^{1} \mathrm{H}^{+}\left({ }^{11} \mathrm{~B}, 2 \alpha\right) \alpha$ reaction for power generation. The appeal in this reaction is in the absence of neutron generation, providing added safety and environmental benefit.

\subsection{Small Scale Fusion Experiments}

All of the previous fusion experiments operate on very large scales. Both in terms of physical scale, and financial scale. There are, however, a growing number of groups investigating fusion on much smaller scales as support to the larger experiment, or for investigation into nuclear processes, or even simply for the development of an "on-off" monoenergetic neutron source. 


\subsubsection{Pyroelectric Fusion}

In the past decade, there has been interest in pyroelectric crystals for development of small, even handheld, neutron and x-ray sources. [10] [29] [30] These systems achieve fusion by using tungsten (or other appropriate material) micro and nanotips electrically connected to pyroelectric crystals to field ionize a hydrogen fill gas, and accelerate the ions at an appropriate target. These crystals, when heated have been shown to develop voltages of as much as $300 \mathrm{keV}$, well high enough to fully ionize a background hydrogen gas and provide the acceleration potential to force deuterons or tritons to fuse in deuterated or tritiated solids. [31]

While effective for generation of a monoenergetic neutron beam, these are not practical in power applications as the pyroelectric crystal depends on the surface charge to provide the acceleration potential. This surface charge is slowly depleted by the accelerated particles, and once depleted, must be regenerated by heating or cooling. The surface charge is typically on the order of a $\mu \mathrm{C}$, and so even if all ionized atoms caused fusion events, it would still be insufficient to generate net positive energy. In addition to this the heating and cooling cycles needed for the generation of the accelerating potential don't provide stable voltages, and there exists a significant spread on the energy of the ions impacting the target. This same principle of simultaneous ionization and acceleration has been applied to fusion without pyroelectric crystals. [32] This system has, however, the limitation of the lack of beam focus that is attainable from ionizing tip acceleration systems.

\subsubsection{Plasma Source}

Given then difficulty around creating a stable beam using pyroelectric crystals, focus was instead turned towards using plasma sources, which are more reliable and stable ion sources. The primary reference points for the ion source developed in this work, is the work done by J. L. Rovey [33] [34] as well as the work done by B. K. 
Das and A. Shyam. [35]. These works were focused on development of a lab neutron source using a PIG as an ion source for the target fusion.

In fusion applications, plasma sources are a common tool for beam extraction and subsequent acceleration into appropriate targets. In addition to that they find use as feed ins for various accelerator and tokamak experiments, making plasma sources a crucial component for much of fusion research. Provided the efficacy and the simplicity they provide, PIG sources continue to be used and developed in modern systems, Though some are as negative ion sources current plasma source experiments, [36] many are as fully ionized proton sources. [34] [35]

\subsection{Previous Experimental System and its Limitations}

The development of the accelerator is an extension of previous work done where an accelerator based on pyroelectric crystals was constructed. In the previous design, pyroelectric crystals were heated and allowed to cool in order to create an accelerating gap of as much as approximately $120 \mathrm{keV}$. This process for generating the ionization and accelerating potential that would accelerate particles into an appropriate target has as a significant drawback the inherent difficulty in maintaining a consistent acceleration potential as the evolved potential for acceleration is dependent on change of temperature for developing more charge. Thus, once the charge that exists on the surface has been depleted, the crystal would need to be heated or cooled further the redevelop or maintain the acceleration potential. In order to have better control over the energy spread of the incoming particles, it was concluded that it would not be appropriate to use pyroelectric crystals as an ion source for the system being developed. Figure 12 shows a cutaway of the first system designed and constructed with an oversized representation of the tungsten mircotip used to field ionize the fill gas attached to one pyroelectric crystal, and the copper target attached to the other, opposite the tip. In addition to the limitations posed by the pyroelectric crystals themselves, as 


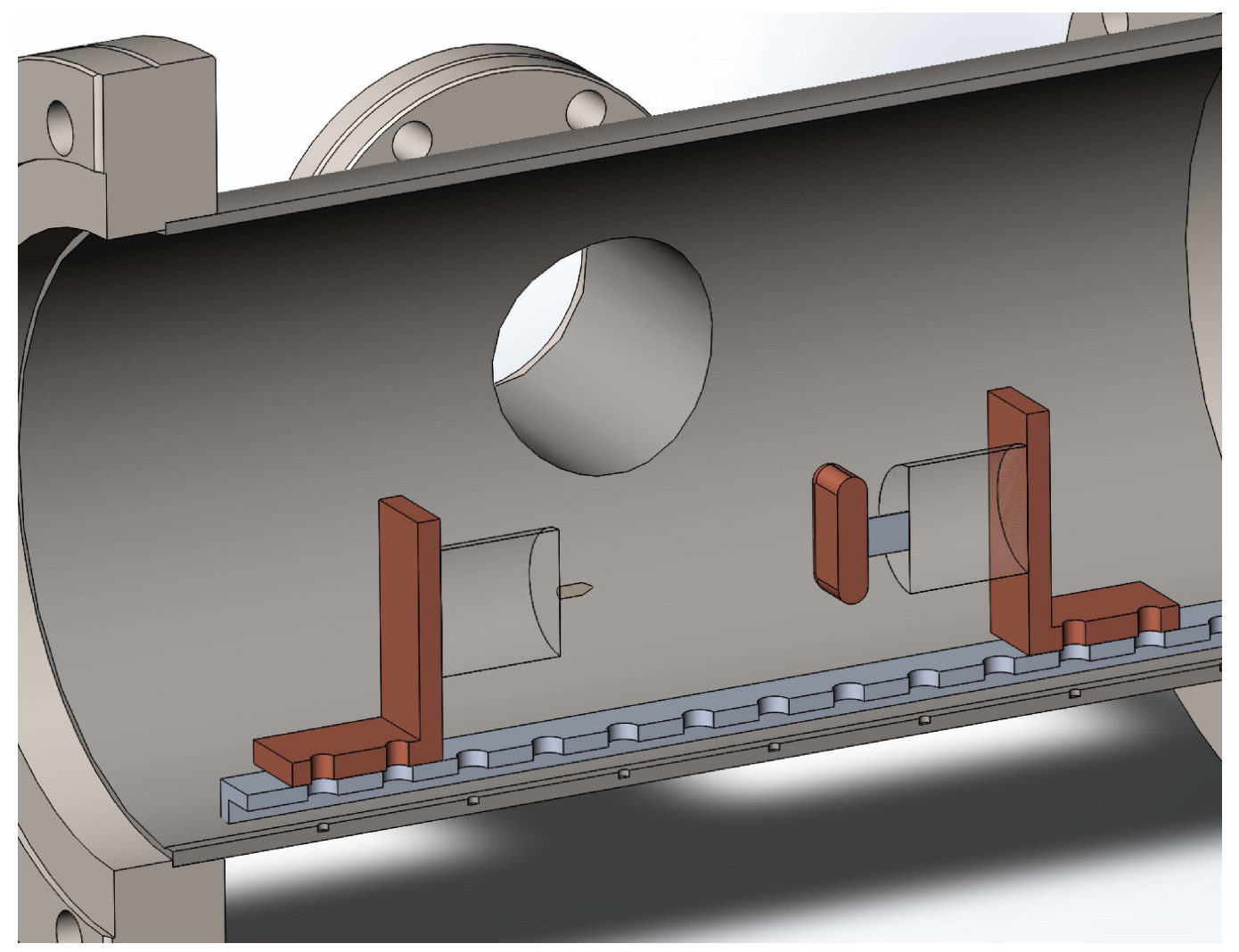

Figure 12: Cut away of the original pyroelectric based accelerator and vacuum chamber

can be seen in Figure 12, the previous design suffered from very limited space. This imposed significant restrictions on diagnostics that could be applied to the experiment. Furthermore, the limited space imposed a limit on the ability of the project to grow and accommodate different techniques that could be used to achieve fusion. 


\section{Design}

\subsection{Initial Considerations}

The accelerator assembly was designed to have a long-term usability, and to allow for the project to evolve and grow as needed. It is for this reason being both self contained and modular were the design goals for the test bed system. To enable accurate design of the external components, the Computer Aided Design (CAD) tool SOLIDWORKS was used to model the external and internal components. Of the available HV components available, the two components with the largest inner volume were a CF8 six-way cross, and a spherical chamber with four CF8 ports, four CF6 ports, four CF2.75 ports, and two CF4.5 ports. These two components, seen in Figure 13 , formed the base of the system. These components were cleaned and prepared for

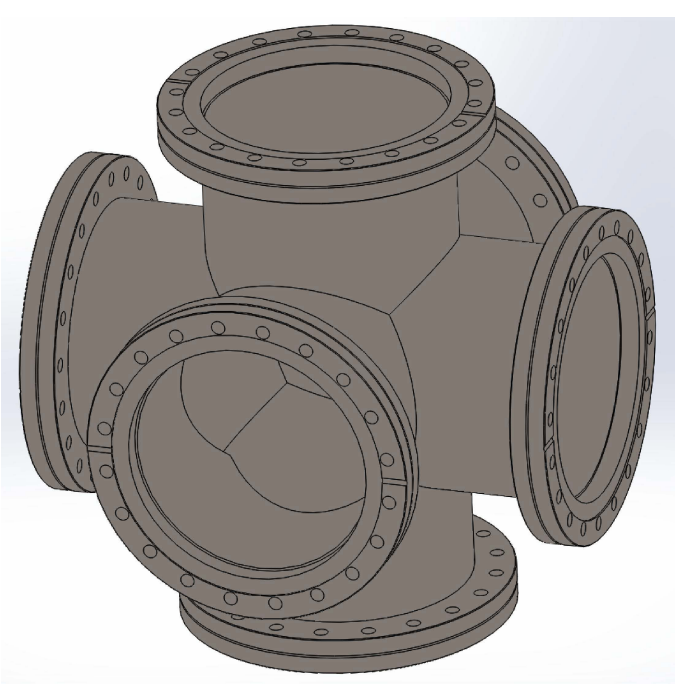

(a) Six way CF8 cross

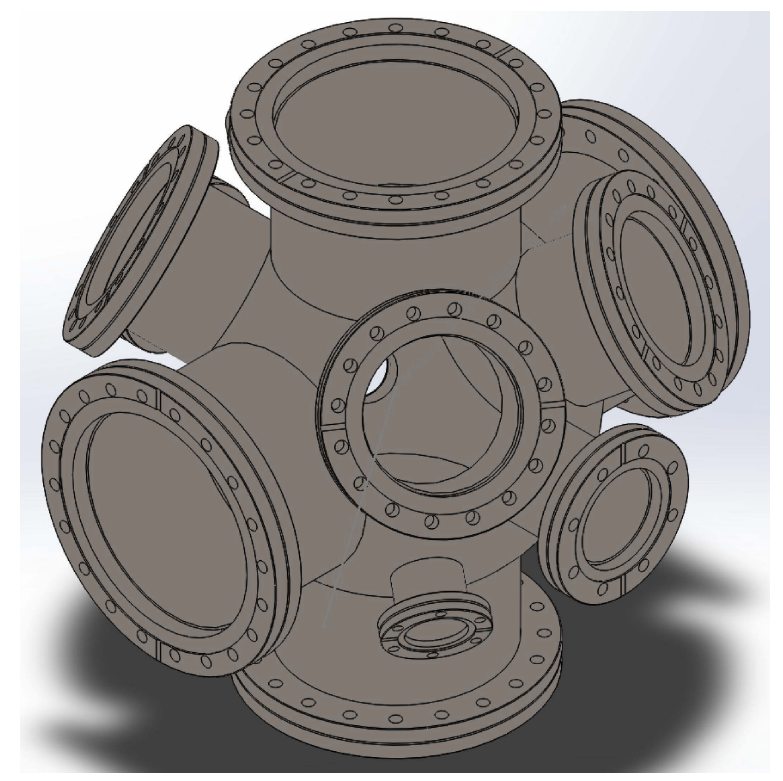

(b) Spherical chamber with CF flanges

Figure 13: Base pieces for modular test bench

use by using scouring pads to remove any visible residue, followed by a thorough rinse with tap water, and a final pass with an isopropanol wetted pad. Based on their configurations, it was decided that the spherical chamber (Figure 13b) would be 
used for the targets, while the cross (Figure 13a) would be for sources. This decision was due largely to the significant number of ports on the sphere that would allow for sophisticated and expanding diagnostics as the project progressed. Following this decision, it was concluded that the best place for the vacuum pump would be directly attached to the source chamber. This is to provide a safety separation between the potentially more volatile target, as well as to improve the overall ultimate pressure of the system. The source would in many scenarios be a plasma source and so would require an inflow of gas to supply the plasma. Having the pump immediately adjacent to the gas source would allow for a lower overall pressure increase in the system. A desirable addition to this system are gate valves between the both the source and target chambers, as well as between the entire system and the vacuum pump. This allows for finer leak detection and isolation as well as the ability to safely perform a fast shutdown if needed. Due to the size of CF8 gate valves and the density of flanges on the spherical chamber, an additional extension piece was used between the target chamber and the gate valve. The starting point for the modular test bed system is seen in Figure 14, and was the base for all further designs, including the ion source and target sub-chamber. This beamline has an inner diameter of approximately $15 \mathrm{~cm}$, and a length of approximately $95 \mathrm{~cm}$. Finally, the support structure that would house the vacuum chamber and its equipment was designed, again with a focus on modularity and future growth. The resultant design is shown in Figure 15. Due to the distance between the target and the source, it was necessary to design electrostatic beam control components to be able to properly maintain a proton beam with a beam width on the order of the target size. Rearranging Equation 2, an equation for the required pressure to give the needed MFP for the ions can be found, as given by Equation 33

$$
P=\frac{k T}{\pi D_{m}^{2} L_{i 1}}
$$




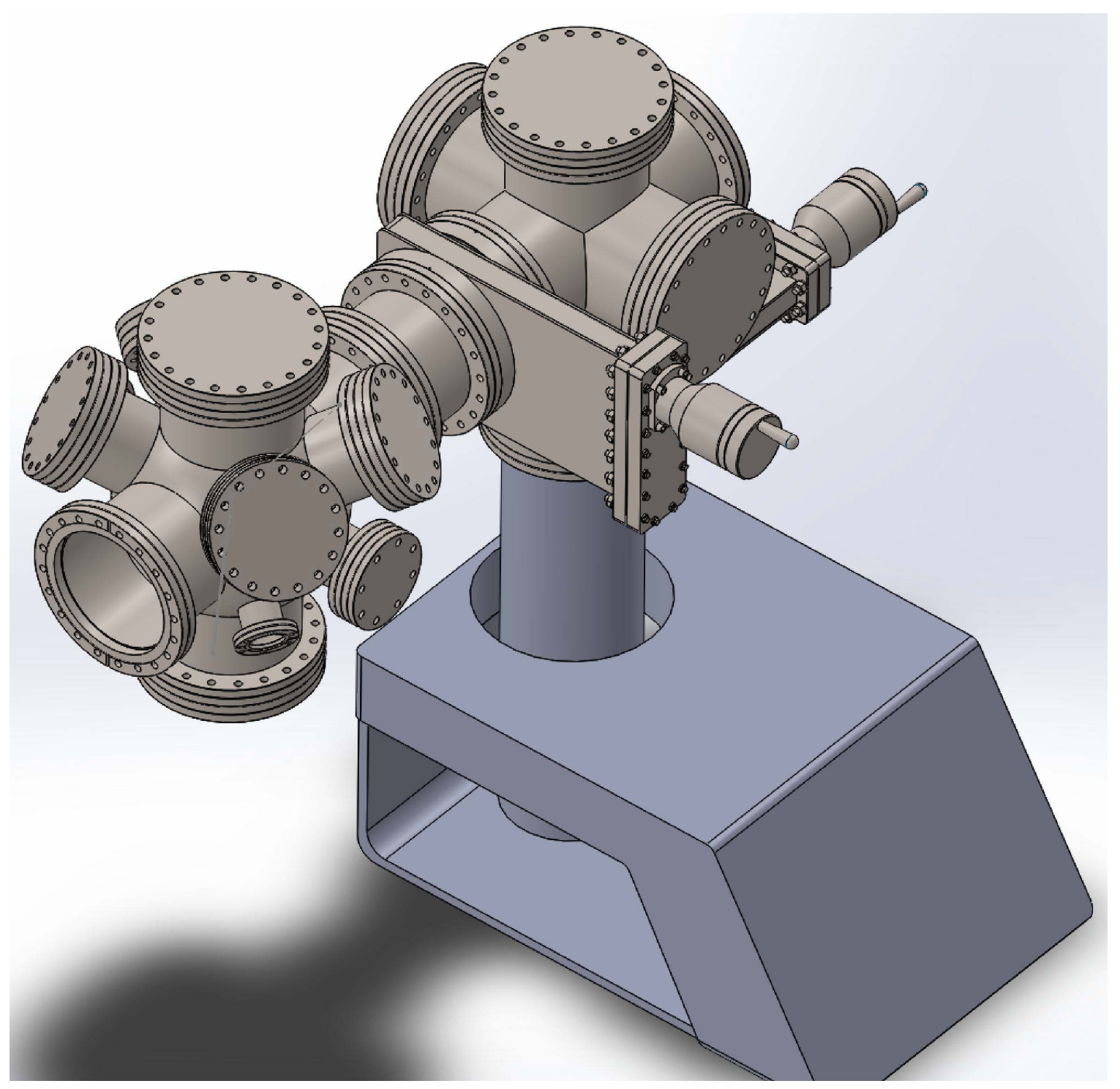

Figure 14: Base beamline showing key components

Assuming a primarily hydrogen background and a proton as the ion travelling through the gas, the required pressure to give a mean free path greater than the $95 \mathrm{~cm}$ distance between both ends of the chamber could be calculated using the data from Table 2 . The required pressure was found to be $<1.76 \times 10^{-2} \mathrm{~Pa}$, or $<1.3 \times 10^{-4}$ Torr. Given the components selected, this pressure was easily attained. The pump selected for use with the system was a turbopump station, from which the pressure gauge controller, diaphragm backing pump, and turbopump were contained and controlled. The diaphragm pump provided the benefit of a small oil-free pump, while the air cooled 


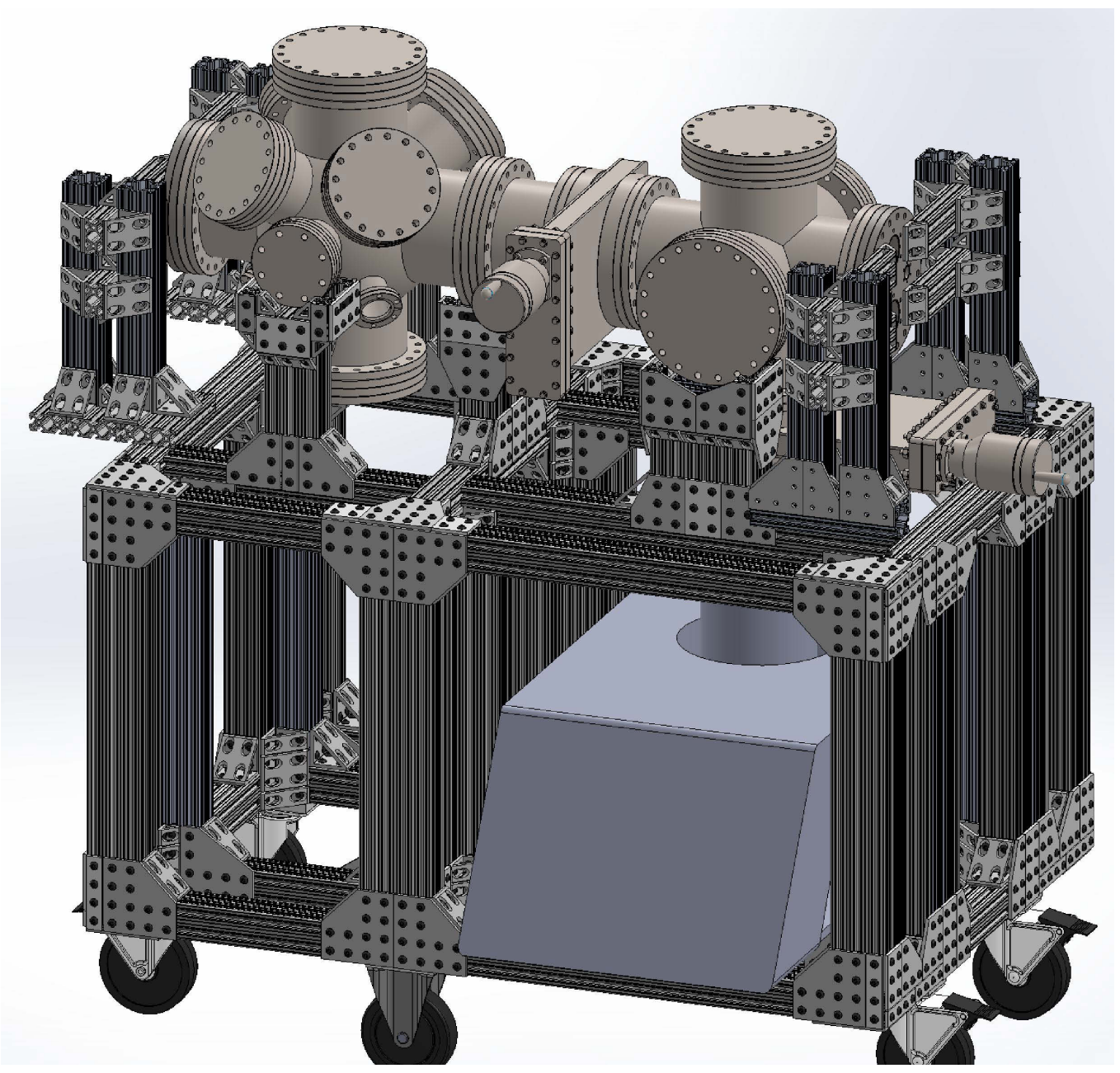

Figure 15: Full assembly CAD design

turbopump provided a simple to use and highly effective pump. Finally, considering the ultimate goal of fusion with a target, an acceleration potential was chosen by inspection of cross-section plots of some reactions of interest. Given that many of the reactions shown in Table 6 have cross sections in the millibarn range $(\mathrm{mb})$ even at $50 \mathrm{keV}$, an acceleration potential of $60 \mathrm{keV}$ was selected as a starting point. This was achieved using two sources of opposite polarity, the benefit being that lower potential simplified the size requirements of the high voltage feedthroughs that would have to be used. 


\subsection{Target Sub-chamber}

The target sub-chamber was a necessary component to enable the use of airreactive targets. The requirement was thus that the target can be kept from contacting air while in transit from a glove box to the chamber itself; and once in the chamber, exposed to the beam without negatively affecting its trajectory. This would mean that the sub chamber when open should be axially symmetric. The glove box used to store the air reactive materials has a load lock of approximately $20 \mathrm{~cm}$, and the vacuum chamber for the beamline has only a linear actuator with a maximum deliverable force of $25 \mathrm{~N}$. Given the described constraints, the sub-chamber designed is shown in Figure 16, and its clearance was confirmed using CAD software as shown in Figure 17. This sub-chamber had two feedthroughs, one and electrical and one

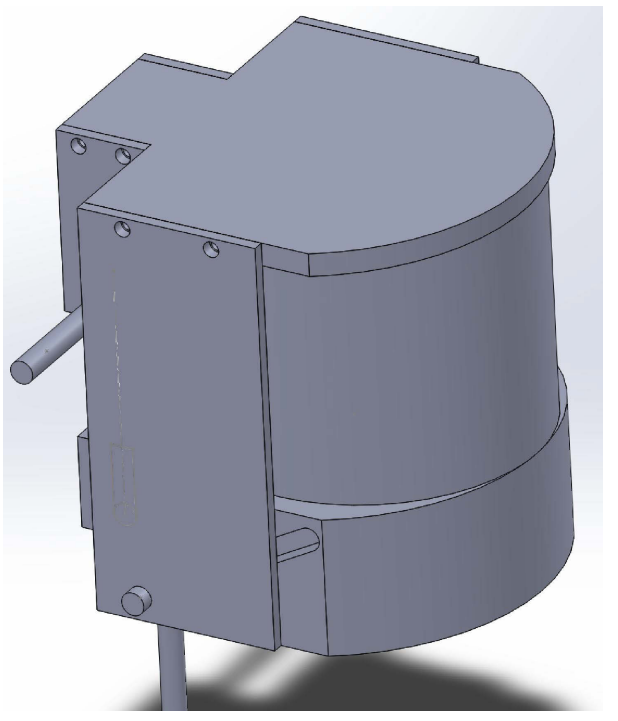

(a) Closed

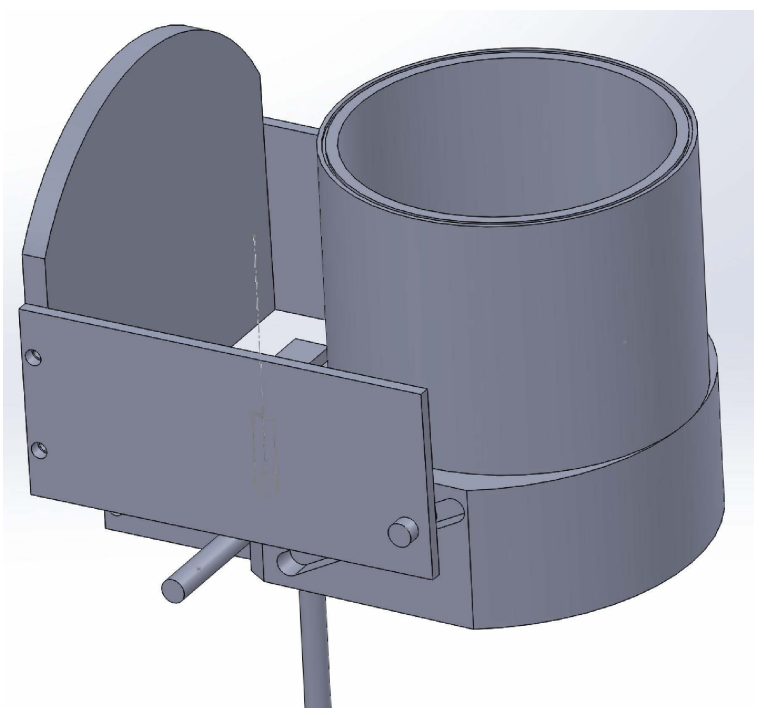

(b) Open

Figure 16: The target sub-chamber

gas. The gas feedthrough was connected to a $7 \mathrm{kPa}$ check valve and allows gas to escape when the pressure outside the sub-chamber is greater than inside. By using the check valve it was possible to have a chamber that would self-seal during the pump down cycle of the glove box load lock; prevent any air from making contact with the sensitive target; and minimizing the force that the linear actuator would 


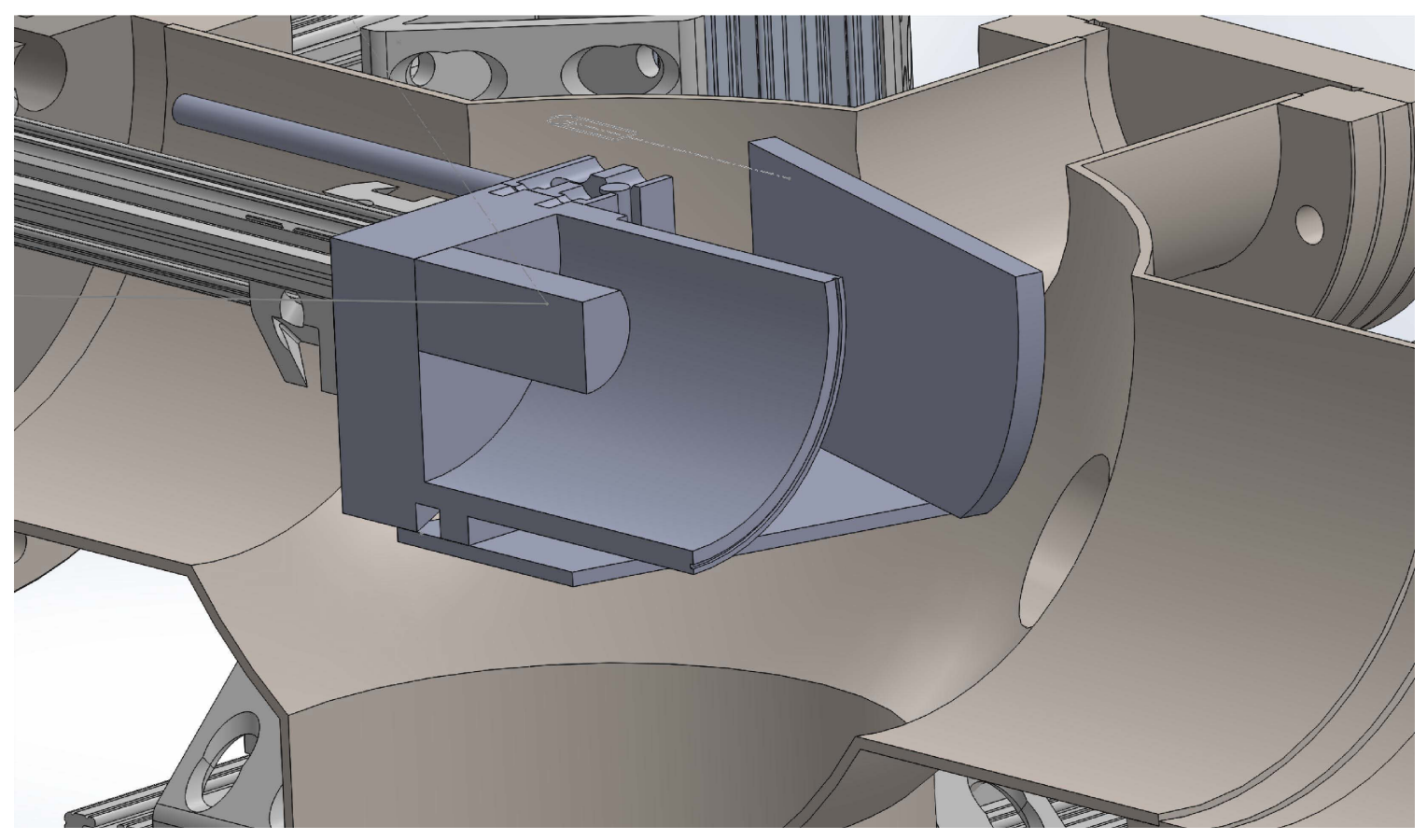

Figure 17: Cutaway of the beamline with the target sub-chamber in place demonstrating clearance

need to apply to expose the target. 


\subsection{Ion Source and Electrostatic Lenses}

\subsubsection{Theoretical Design of the Ion Source}

As previously mentioned, a starting point for the design of the PIG were the ion source designed by J.L. Rovey, [33] [34] and the source designed by B.K. Das and A. Shyam [35]. Their designs were both cold cathode Penning type ion sources with a focus on simplicity, compactness, and affordability. A PIG was ultimately chosen as the first ion source because of the flexibility provided by a cold cathode DC source, and simpler construction than a more sophisticated RF source or other DC source. Ultimately, this meant that it was possible to build with little more than access to a lathe. This was particularly desirable for cost considerations, as well as maintainability moving forward.

The source designed by Rovey made use of a Samarium cobalt (SmCo) disc magnet affixed to the base of a bored-out section of iron, onto which the plasma electrode was affixed. The suggestion being that this was done to create the necessary magnetic field for support of the plasma source. Due to the greater availability of Neodymium iron boron $(\mathrm{NdFeB})$ magnets, and significantly lower cost, a simulation was carried out in COMSOL Multiphysics (COMSOL) to investigate the magnetic field achieved with a single $\mathrm{NdFeB}$ magnet in a non-magnetic material. Figure 18 shows the results of the magnetic simulation using both a ring magnet around the anode, the ring magnet and a disc magnet, and just a disc magnet below it. As demonstrated by the simulations, the field inside the anode is in the range of $0.3 \mathrm{~T}$ which as was indicated in [19] is within the required $0.1 \mathrm{~T}$ field. As such, was deemed a sufficient field for the PIG source.

This simulation was done separately from the ion optics, as IBSIMU, the ion optics simulation library, was unable to generate its own magnetic field simulations, but was able to simulate the effect of magnetic fields on ion beams. Thus, the results for these 


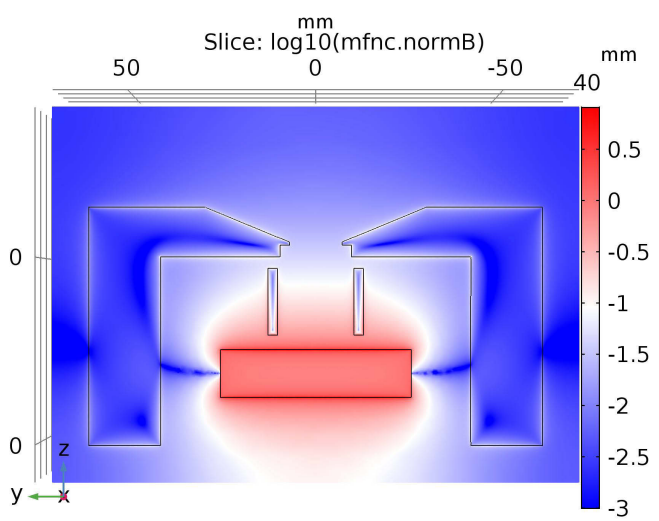

(a) PIG with disc magnet

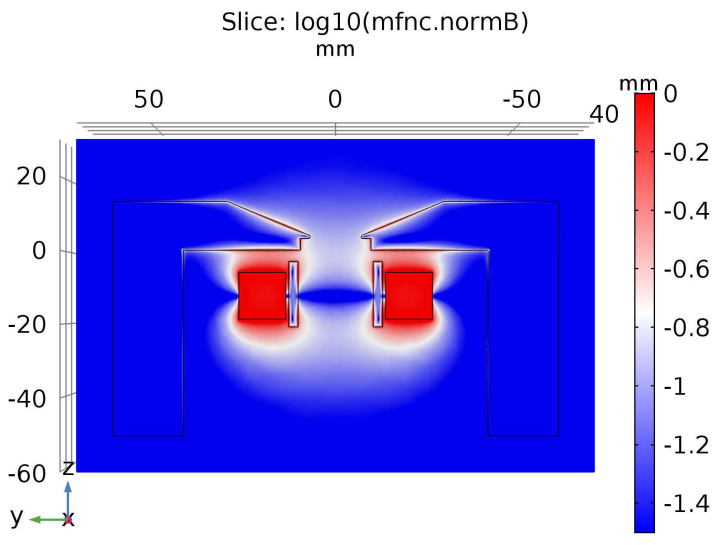

(b) PIG with the anode ring magnet

Figure 18: Results of COMSOL magneto-static simulations $\left(\log \left(\frac{B}{1 \mathrm{~T}}\right)\right)$

magneto-static simulations were subsequently fed into the ion optics simulations. The design of the extraction electrode was carried out using the equations described in section 2.3.1, and once the radius was found, it was modified to be a flat section. This is done in order to reduce the manufacturing difficulty, and still maintain a nearly flat plasma meniscus. [16] In determining the radius for the circle, the two values that are required are the electron number density of the plasma and the electron temperature, $n_{e}$ and $T_{e}$ respectively. For a PIG ion source, the $\frac{k T_{e}}{e}$ was taken as being $5 \mathrm{eV}$ based on data from [37] and corroborated by the range found in [38]. The assumed value of $n_{e}$ was between $1 \times 10^{16} \mathrm{~m}^{-3}$ and $1 \times 10^{18} \mathrm{~m}^{-3}$, as given in [16]. Using these values, the radius of the circle for the extraction electrode is on the order of $1 \times 10^{-4} \mathrm{~m}$ or less, a feature size that would present significant challenges in manufacturing. As such, the lip was dropped altogether and the design investigation was turned to focus on the effect of varying the extraction electrode angle. As a rule of thumb, due to erosion from sputtering, manufacturing limitations, and other effects, a practical lower limit to the thickness of a plasma electrode is $0.2 \mathrm{~mm}$. [39]. This was further mitigated by manufacturing the plasma electrode from 304-SS, while using aluminum for the remaining body components. The final design, however, had a safety factor of three applied to the thickness, bringing the designed value to $0.6 \mathrm{~mm}$. The resultant 
electrode and the overall PIG source are shown in Figure 19.

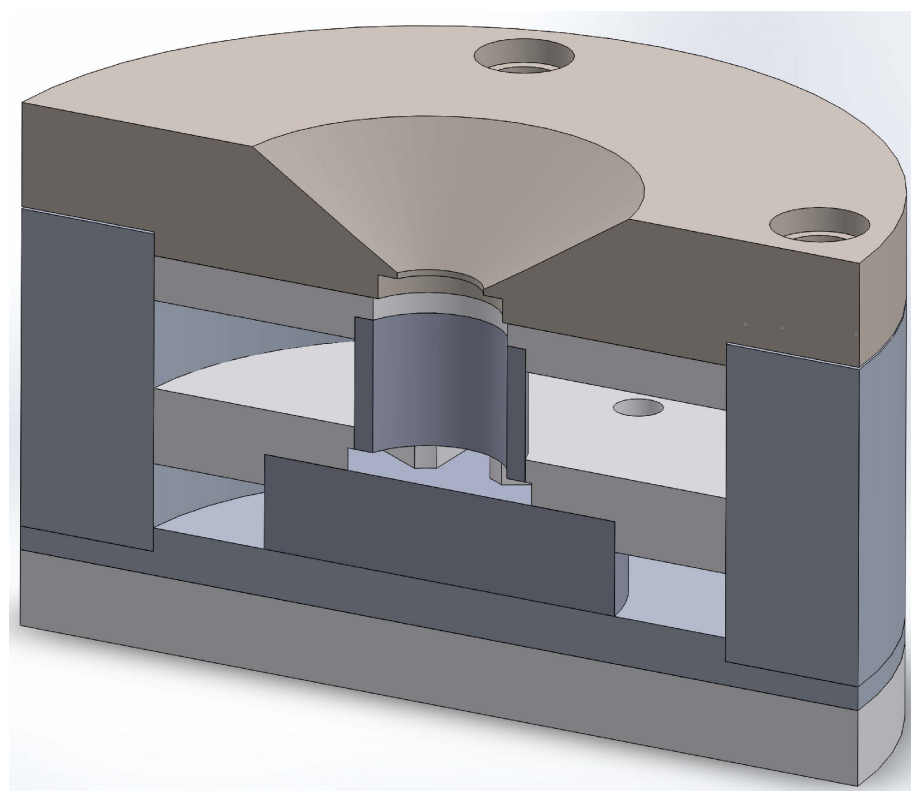

Figure 19: CAD design of PIG source

\subsubsection{Theoretical Design of the Electrostatic Lenses}

Having designed a beamline that was as long as $95 \mathrm{~cm}$, it was necessary to make use of ion optics to control the spread of the beam. Figures $24 \mathrm{a}$ and $24 \mathrm{~b}$ demonstrate the spread that the beam would undergo with no ion optics elements. The electrostatic lens design had to consider limited space and limited range of potential that could be applied to them. Using the matrix equations described in section 2.3.2 in a Python script (shown in appendix A.3.1) the appropriate position and potential were found iteratively. In the design, despite the benefits provided by using the accelerating Einzel configuration, a decelerating configuration was used for the higher refractive power it provided. This paper design, however, would not accurately account for fringe effects that would be caused by the low ratio of aperture diameter to electrode separation, and so the values calculated were later refined using the custom ion beam simulation suite developed for this work, and described in section 4.3.3. To allow for further refinement of the positioning of the electrodes, the system for mounting 
them made use of small separation beads that would allow for the fine and repeatable adjustment of the electrode positions. The whole assembly including the mounting flange and PIG is shown in Figure 20.

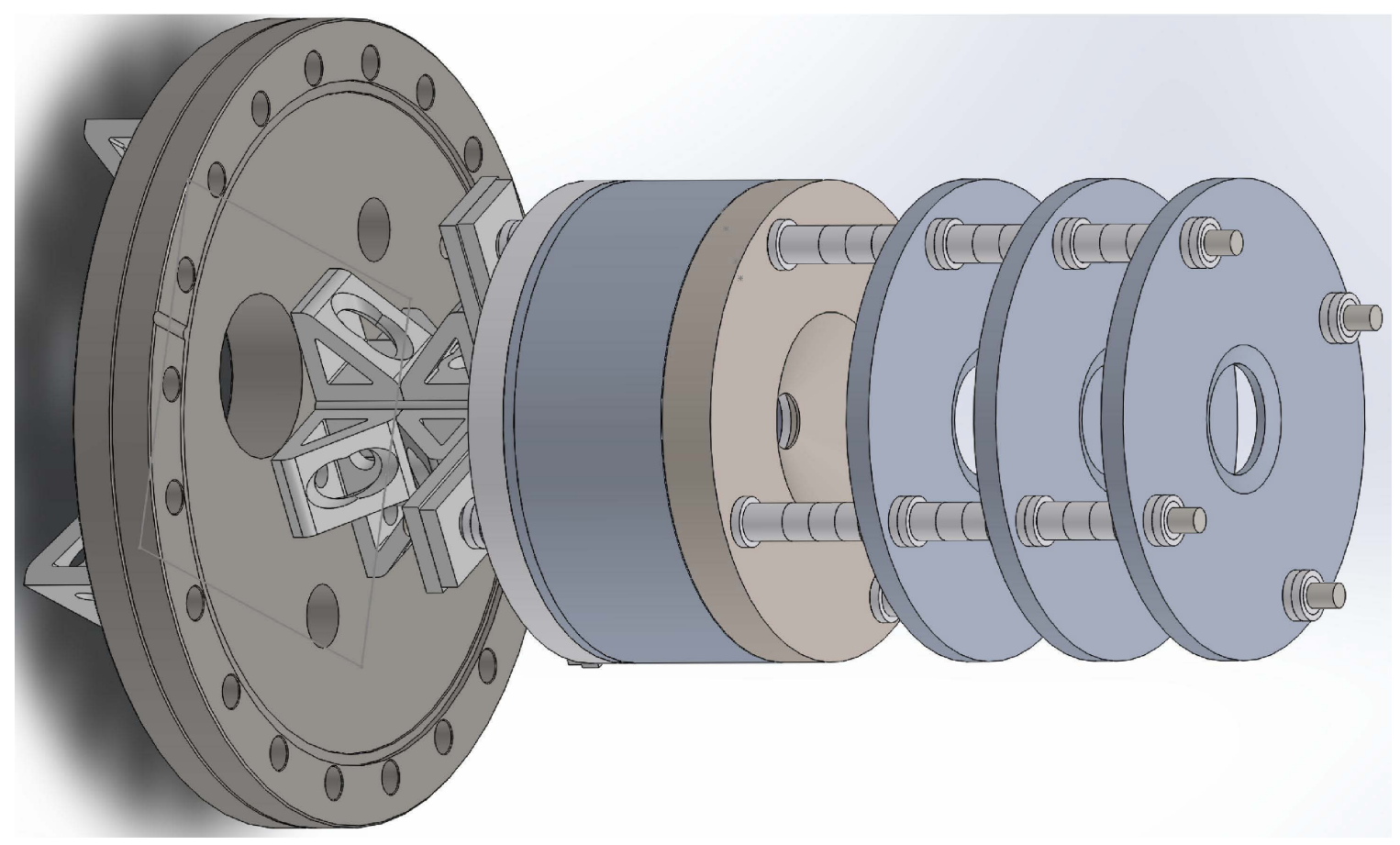

Figure 20: CAD design of PIG with Einzel lens and mounting flange

\subsubsection{Simulation of Beam Extraction and Optics}

The ion optics simulations were, as previously stated, done using the $\mathrm{C}++$ simulation library IBSIMU. [40] IBSIMU was chosen over similar tools available in multiphysics software like COMSOL or ANSYS because the latter two do not have the ability to simulate space-charge effects, a crucial component of plasma extraction and ion beam steering simulations. Beyond the immediate feature options, the use of an open source $\mathrm{C}++$ library provided greater transparency into the code's operation, as well as greater modularity, extendability, and improved data collection from simulations. The simulation suite that used the IBSIMU libraries was written in Python for its ease of use, expandability, and access to modern scientific packages. A key feature 
that was added to this was the ability to define multiple simulations, each with their own run parameters, and to run them in parallel to one another. This greatly accelerated the iterative process that was employed in finalizing geometry designs, and optimal parameter seeking.

In order to properly track all the results of the simulations, the data was fed into a SQL (Structured Query Language) database implemented using PostgreSQL. It is, however, important to note that while IBSIMU can run simulations with imported magnetic fields, it cannot create the simulations itself, and so the magnetic field data was created using COMSOL as described in 4.3.1. An additional feature added to the simulation suite was the ability to split simulations into sub-simulations, where the particles would stop at an artificial barrier, and be reloaded into the next section of the simulation where the electric field would be solved for a different region. The purpose of this was to allow for different sections to be run with different geometry constraints and at different resolutions.

For example, in the main beamline where the distance between electrodes is greater, it is desirable to include much more of the geometry, including the chamber walls which are axially asymmetric to capture the geometry's effect on the electric fields. In the region of plasma extraction, however, a much higher resolution is needed to enable the simulation of the plasma extraction, and to capture the smaller features of the plasma electrode. This was the scenario that necessitated this feature originally. After implementation, however, it was discovered that the electric fields varied too greatly for each sub-simulation when compared against a full simulation. This was a result of the loss of the "next-nearest-neighbour" influence of the electrodes. This feature was left without the ability to include the next nearest neighbour in the electric field because the feature size of the simulations was on the order of the maximum practical resolution of the simulation, and as such conducting full system simulations was a viable option, and chosen as the best course of action. 
The IBSIMU library uses the finite difference method to discretize two coupled equations and solve the ion trajectories: Poissons formula to described the interaction of space-charges and electric fields, shown in Equation 34; and the time independent Vlasov equation to solve the interaction of the particle distribution with the resultant electric field and the imported magnetic field, shown in Equation 35.

$$
\begin{gathered}
\nabla^{2} \phi=-\frac{\rho}{\varepsilon_{0}} \\
\vec{\nu} \cdot \nabla f-\frac{q}{m}(\overrightarrow{\mathcal{E}}+\vec{\nu} \times \vec{B}) \frac{\partial f}{\partial \vec{\nu}}=0
\end{gathered}
$$

Where $\phi$ is the self-consistent electric potential $(\mathrm{V}), \rho$ is the space-charge $\left(\mathrm{C} \mathrm{m}^{-3}\right), \nu$ is the particle velocity $\left(\mathrm{m} \mathrm{s}^{-1}\right), f$ is the particle distribution function $\left(\mathrm{Cs} \mathrm{m}^{-4}\right), q$ is the particle charge $(\mathrm{C}), m$ is the particle mass $(\mathrm{kg}), \mathcal{E}$ is the electric field $\left(\mathrm{V} \mathrm{m}^{-1}\right)$, and the $B$ is the magnetic field $(\mathrm{T})$.

These equations, when formed in the three dimensional case as used in this work, are solved using iterative stabilized biconjugate gradient method. In the same work in which it was introduced, the IBSIMU library was also validated and shown to produce results that closely matched to real world results on a $\mathrm{H}^{-}$plasma extraction scheme. [15]

To achieve meaningful results, simulation parameter selection is critical; particularly important parameters are simulation resolution, space-charge under-relaxation, and the error for convergence. Space-charge under-relaxation is of particular importance when performing plasma extraction simulations. This resolves stiffness in the solution by forcing smaller steps sizes on the iterative space-charge calculations, slowing convergence, but preventing oscillation between two solutions that can occur as a result of the system trying to compensate for too high space charge, and subsequently too little space charge. The resolution for the simulations was determined based on 
minimum feature size. This was then verified by running simulations of resolutions higher and lower than the minimum feature size. Because the simulations were 3D, the memory consumption would increase following a cubic relation and would quickly become larger than the available memory on the computer host. As such it was concluded that due to the minimum feature size of $0.6 \mathrm{~mm}$, this was sufficient resolution to allow for quick simulations, without losing information. For these simulations, resolution selectrion was critical because at the resolution of $0.6 \mathrm{~mm}$, simulation time was approximately six to seven hours. At high resolution this simulation time could reach 24 hours, and in some cases 48 hours.

Initial simulations carried out investigating different extraction electrodes are seen in Figure 21. Visible in green are the equipotential field lines for between the plasma electrode (shown in blue at the bottom of the plot), and the first Einzel lens electrode (also in blue at the top of the plot); the red lines are the representative particle trajectories and the direction of travel is upwards from the bottom of the plot to the top. As can be seen, the variation in the angle between $57.5^{\circ}$ and $72.5^{\circ}$ has little effect on the extracted beam. However the flat electrode does change the beam characteristics noticeably. All the extracted beams, however, are strongly divergent. As described in section 2.3.1, the Child-Langmuir equation defines the current density at the extraction plane where the space-charge of the beam will limit the extracted current. As such, geometries that flatten the extraction electrode equipotential lines are dependent on the system operating close to the Child-Langmuir limit. For the parameters used in the simulation shown in Figure 21, the current density was on the order of $6.5 \mathrm{~A} \mathrm{~m}^{-2}$, well below the limit on the order of $300 \mathrm{~A} \mathrm{~m}^{-2}$. This results in insufficient "pushback" against the applied electric field, and a divergent extracted beam. In order to address this, recalling the Child-Langmuir Equation 15, the distance between the first Einzel electrode and the plasma electrode was increased, and the potential between them reduced until the space-charge limit current density was 
approximately $30 \mathrm{~A} \mathrm{~m}^{-2}$.

The results of the modified geometry and potential is seen in the plot of Figure 22. As with the previous set of extraction images, the extraneous red lines are particles whose trajectories were made to look artificially angular due to the compression to 2D and are simply lost due to collisions with the electrodes. Examining the emittance plots for the different extraction geometries, it is clear that the majority of the extraction geometries result in a focused beam, except for the flat extraction geometry, which gives a more divergent beam. This is shown clearly in the comparison of the emittance ellipses of the different extraction geometries, seen in Figure 23. The black ellipse in the plots are the fitted emittance ellipse, which encloses $90 \%$ of the beam particles. As shown in Figure 23 the $y$ is the position of the particle along the $y$-axis, and $y^{\prime}$ is the the ratio of the $y$-momentum, and the $z$-momentum, where the $z$-axis is the direction of beam travel. The increasing angle results in the fitted ellipse gradually slanting and becoming more oblong, pointing towards greater divergence.

With the design parameters of the first two electrodes selected, the entire beamline was simulated to compare the extraction electrode angles, as well as to determine the required Einzel electrode separation, and potential. The results of the first pass simulations are shown in Figure 24, and tabulate quantitatively in Table 7. As before

Table 7: Tabulated currents for different extraction geometries

\begin{tabular}{c|c|c|c|c|c}
$\begin{array}{c}\text { Extraction } \\
\text { Angle } \\
\mathbf{(}^{\circ}\end{array}$ & $\begin{array}{c}\text { Additional } \\
\text { Electrodes }\end{array}$ & $\begin{array}{c}\text { Extracted } \\
\text { Current } \\
(\mathrm{mA})\end{array}$ & $\begin{array}{c}\text { Lost } \\
\text { Current } \\
(\mathrm{mA})\end{array}$ & $\begin{array}{c}\text { Current to } \\
\text { Target Holder } \\
(\mathrm{mA})\end{array}$ & $\begin{array}{c}\text { Current to } \\
\text { Target } \\
(\mathrm{mA})\end{array}$ \\
\hline 62.5 & None & 1.38 & 0.69 & 0.52 & 0.17 \\
\hline 62.5 & One & 1.17 & 0.27 & 0.64 & 0.26 \\
\hline 57.5 & Einzel & 0.96 & 0.26 & 0.31 & 0.39 \\
\hline 62.5 & Einzel & 1.06 & 0.26 & 0.42 & 0.38 \\
\hline 67.5 & Einzel & 1.15 & 0.26 & 0.51 & 0.38 \\
\hline 72.5 & Einzel & 1.24 & 0.28 & 0.58 & 0.38 \\
\hline 90 & Einzel & 1.57 & 0.71 & 0.51 & 0.34
\end{tabular}

with the plasma extraction simulations, the ion beam (in red) travels upwards from 
the bottom of the plot, through the Einzel lens (or single aperture), and to the target at the top. As anticipated, the paper design was insufficient to accurately describe the designed geometries due to the aperture-separation ratio assumptions made by the matrix method. This assumption allows the fields between the electrodes to be treated as uniform, but when it doesn't hold true, the fields become warped by the "bulging" from the aperture. [22] In the designed Einzel lens, the aperture diameter is $2.8 \mathrm{~cm}$ and the separation of the electrodes (while variable) is on the order of a few centimeters. In order to narrow down the beam and bring most or all of the extracted current to the target itself and not just the target holder, an iterative process was done where the fields of the lens and the electrode distance were varied. The result of this process is shown if Table 8 and Figure 25, where the extraction electrodes and the resultant beam are compared. From the comparison of the electrodes in the focused

Table 8: Tabulated currents for different extraction geometries

\begin{tabular}{c|c|c|c|c}
$\begin{array}{c}\text { Extraction } \\
\text { Angle } \\
\left({ }^{\circ}\right)\end{array}$ & $\begin{array}{c}\text { Extracted } \\
\text { Current } \\
(\mathrm{mA})\end{array}$ & $\begin{array}{c}\text { Lost } \\
\text { Current } \\
(\mathrm{mA})\end{array}$ & $\begin{array}{c}\text { Current to } \\
\text { Target Holder } \\
(\mathrm{mA})\end{array}$ & $\begin{array}{c}\text { Current to } \\
\text { Target } \\
(\mathrm{mA})\end{array}$ \\
\hline 57.5 & 0.96 & 0.26 & 0 & 0.70 \\
\hline 62.5 & 1.06 & 0.26 & 0 & 0.80 \\
\hline 67.5 & 1.15 & 0.26 & 0 & 0.89 \\
\hline 72.5 & 1.24 & 0.27 & 0 & 0.97 \\
\hline 90 & 1.57 & 0.6 & 0.03 & 0.94
\end{tabular}

system, it was concluded that while the greatest current to the target was achieved with the Pierce angle extractor, the preferred extraction electrode would be the $62.5^{\circ}$ electrode as it would provide a cleaner, less divergent beam that could potentially better withstand non-idealities and variation in the real world system.

To further ensure the selected design was attaining the desired outcomes, the energy distribution at the target was plotted, along with the emittance plot and XYprofile of the beam immediately after the Einzel lens exit. This is shown in Figure 26. 


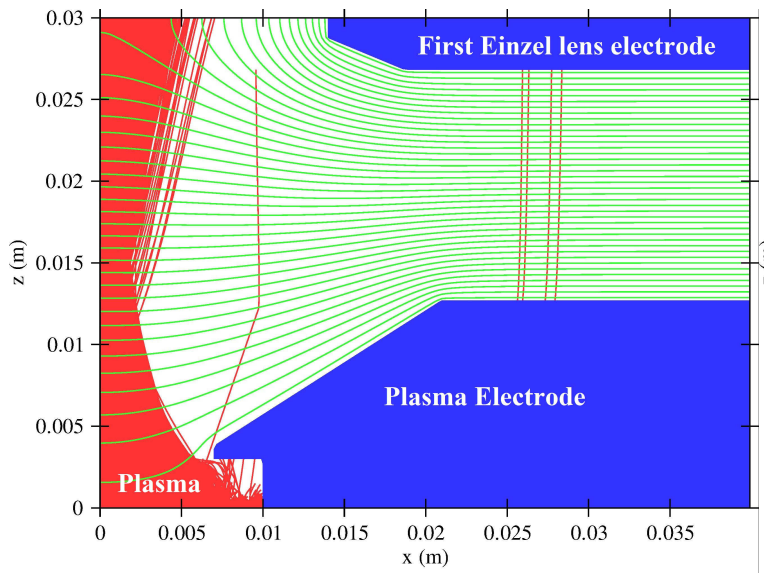

(a) $57.5^{\circ}$

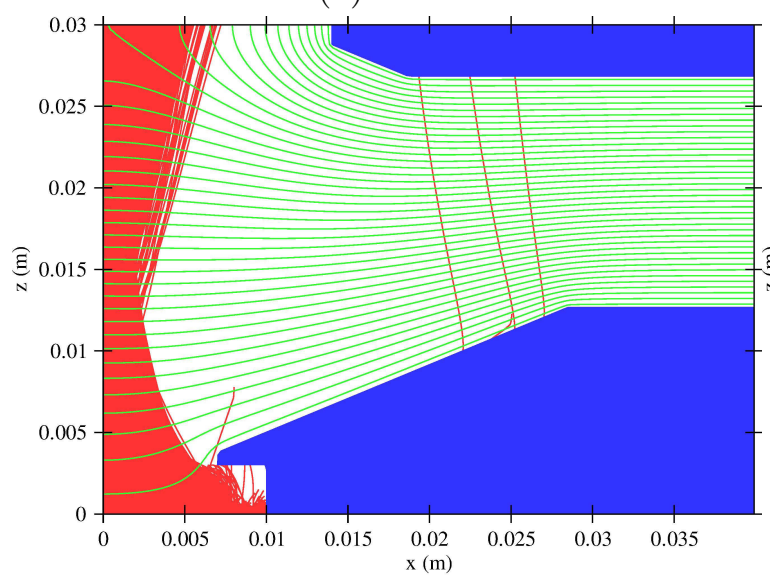

(c) $67.5^{\circ}$

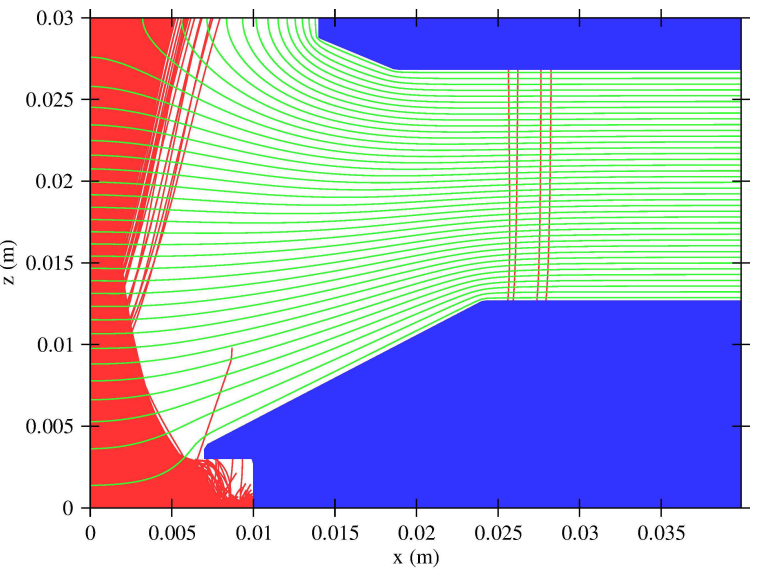

(b) $62.5^{\circ}$

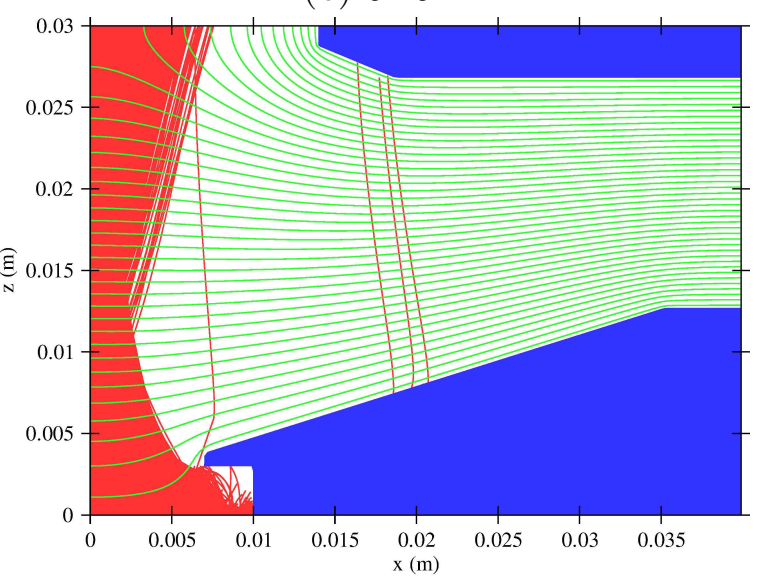

(d) $72.5^{\circ}$

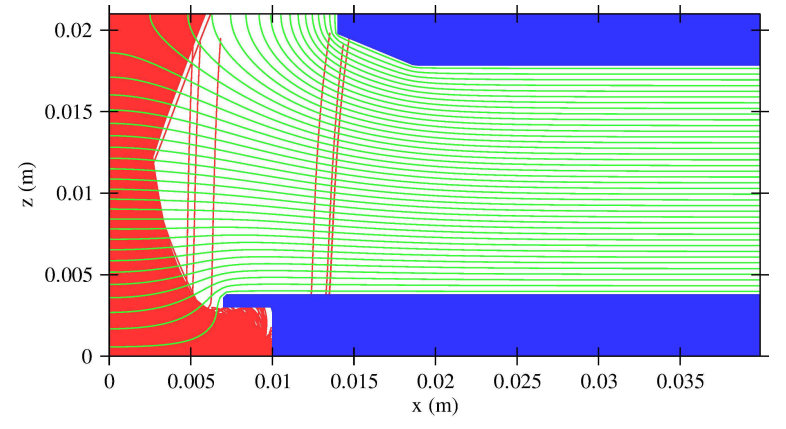

(e) $90^{\circ}$

Figure 21: Comparison of extraction angles and visualization of meniscus potential where the $z$-axis is the direction of the ion beam travel, and the $x$-axis is the distance from the center of the beam. The system is nearly axially symmetric about $z$ 


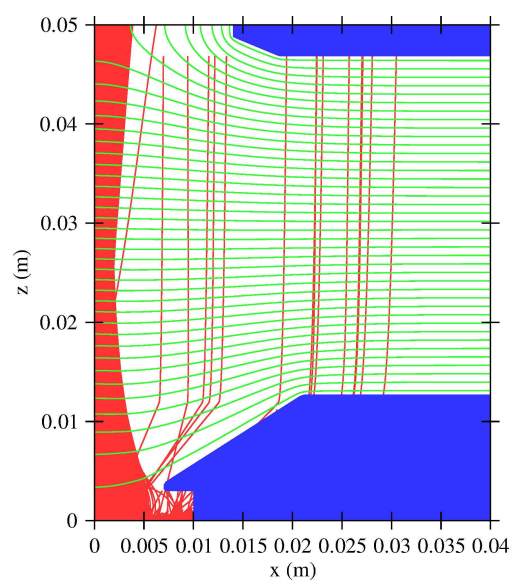

(a) $57.5^{\circ}$

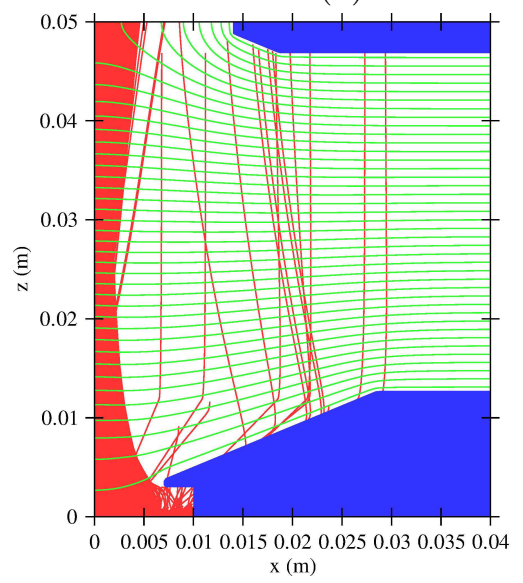

(c) $67.5^{\circ}$

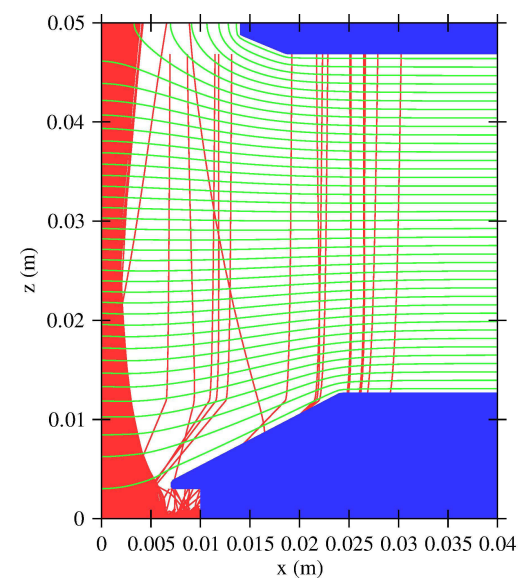

(b) $62.5^{\circ}$

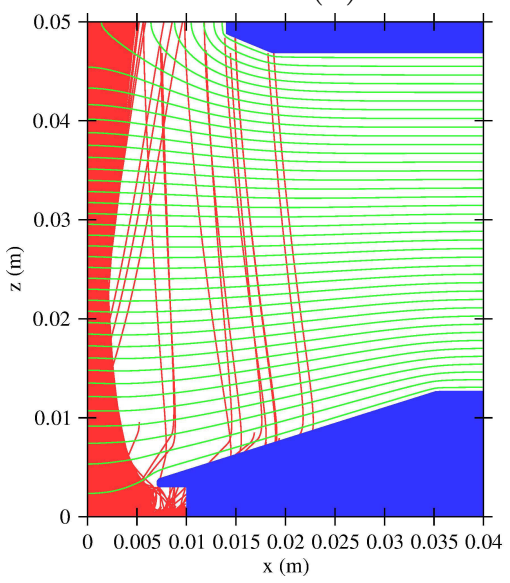

(d) $72.5^{\circ}$

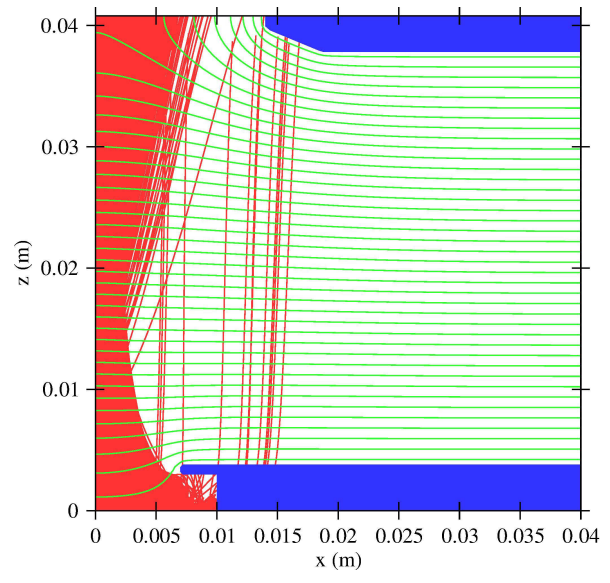

(e) $90^{\circ}$

Figure 22: Demonstration of flattening of meniscus equipotential 


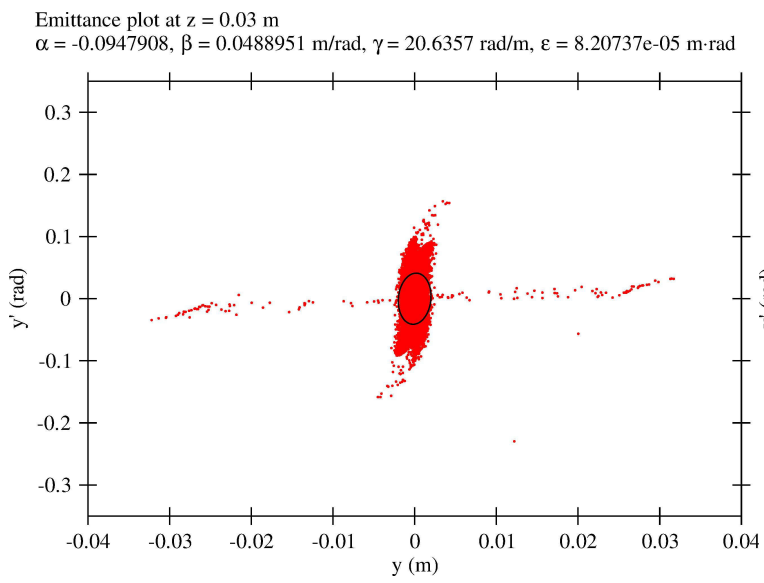

(a) $57.5^{\circ}$

Emittance plot at $\mathrm{z}=0.03 \mathrm{~m}$

$\alpha=-0.155531, \beta=0.0284681 \mathrm{~m} / \mathrm{rad}, \gamma=35.9767 \mathrm{rad} / \mathrm{m}, \varepsilon=7.4388 \mathrm{e}-05 \mathrm{~m} \cdot \mathrm{rad}$

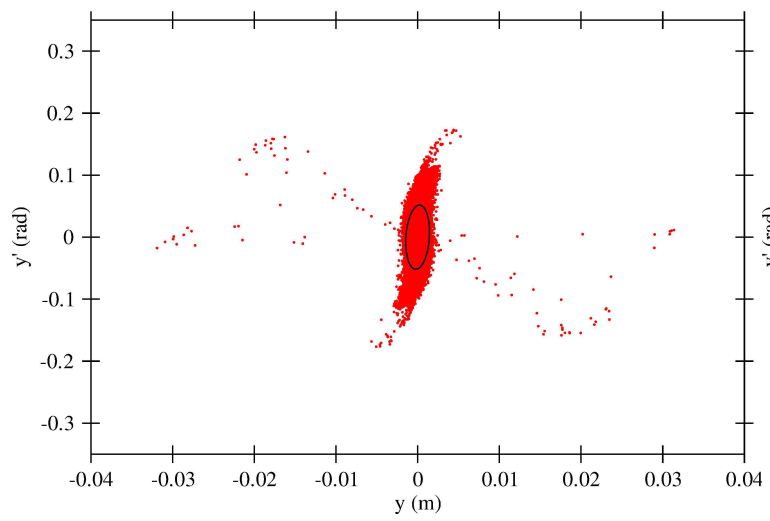

(c) $67.5^{\circ}$
Emittance plot at $\mathrm{z}=0.03 \mathrm{~m}$

$\alpha=-0.115889, \beta=0.0410921 \mathrm{~m} / \mathrm{rad}, \gamma=24.6624 \mathrm{rad} / \mathrm{m}, \varepsilon=8.58641 \mathrm{e}-05 \mathrm{~m} \cdot \mathrm{rad}$

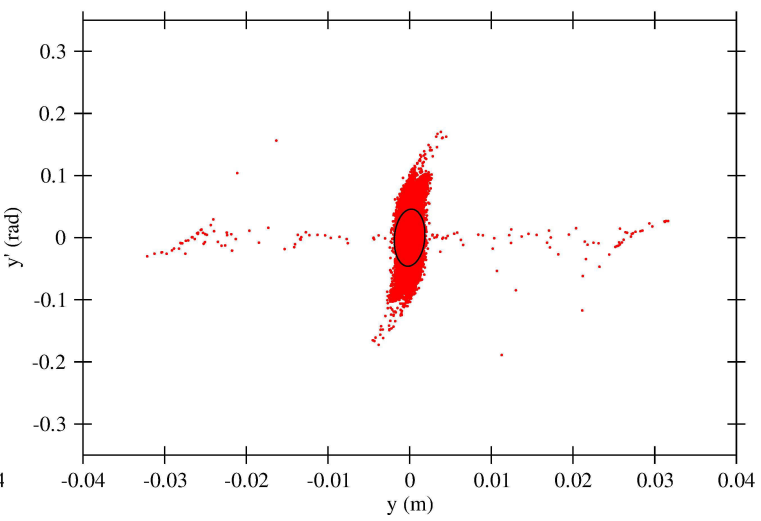

(b) $62.5^{\circ}$

Emittance plot at $\mathrm{z}=0.03 \mathrm{~m}$

$\alpha=-0.308707, \beta=0.0222063 \mathrm{~m} / \mathrm{rad}, \gamma=49.3238 \mathrm{rad} / \mathrm{m}, \varepsilon=6.70127 \mathrm{e}-05 \mathrm{~m} \cdot \mathrm{rad}$

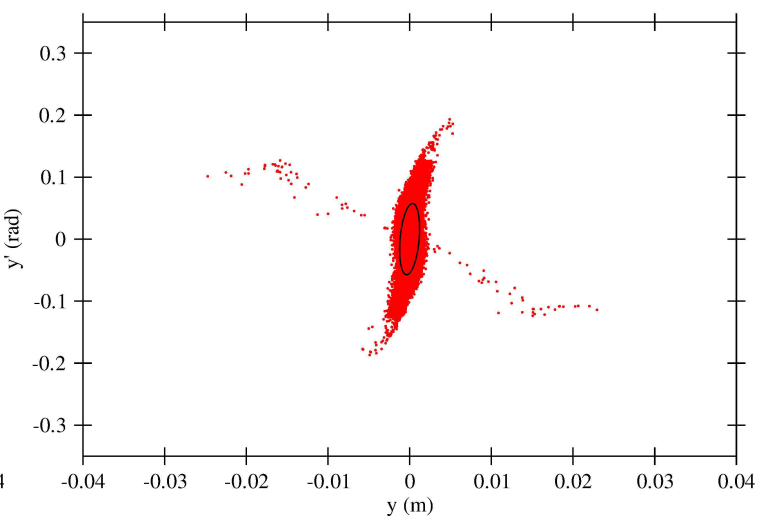

(d) $72.5^{\circ}$

Emittance plot at $\mathrm{z}=0.021 \mathrm{~m}$

$\alpha=-0.351394, \beta=0.0117473 \mathrm{~m} / \mathrm{rad}, \gamma=95.637 \mathrm{rad} / \mathrm{m}, \varepsilon=0.000121814 \mathrm{~m} \cdot \mathrm{rad}$

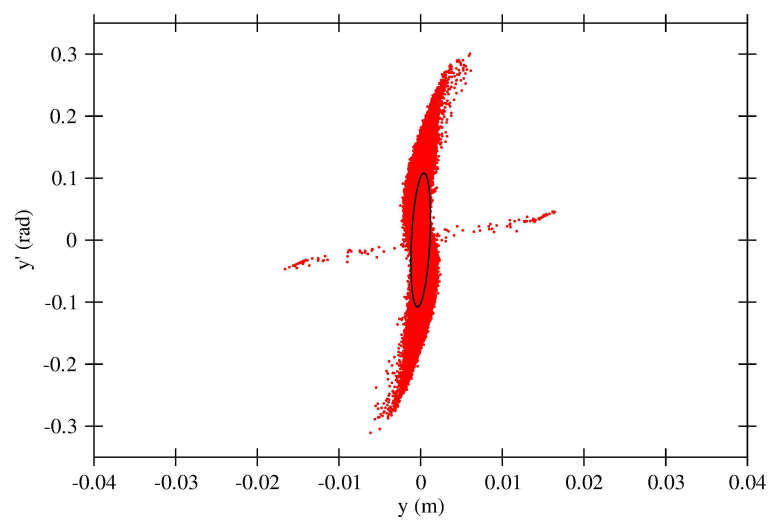

(e) $90^{\circ}$

Figure 23: Emittance plots for the extracted beam taken between the first Einzel lens electrode and the plasma electrode; where $y$ is the distance from the beam center, travelling along the $z$-axis. The system is nearly axially symmetric about $z$ 


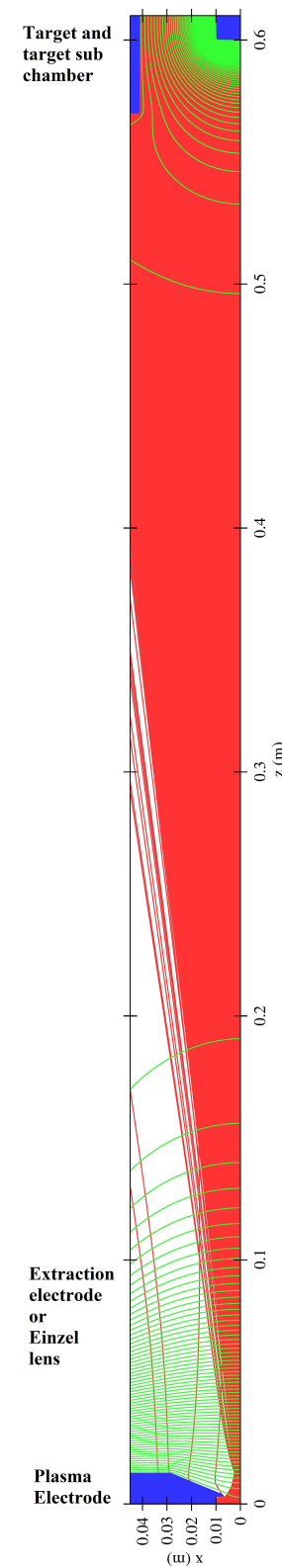

(a) $62.5^{\circ}$

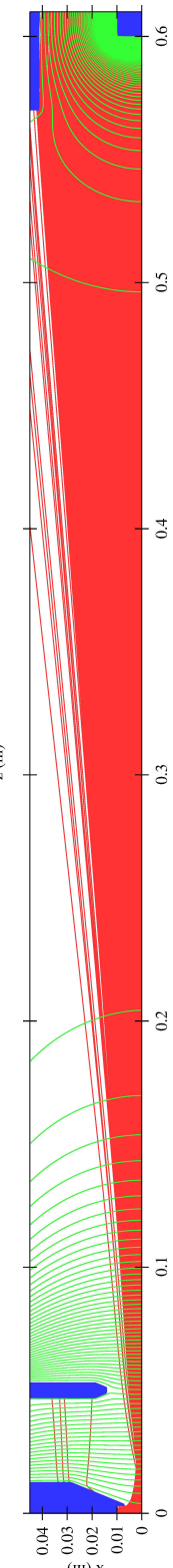

(b) $62.5^{\circ}$

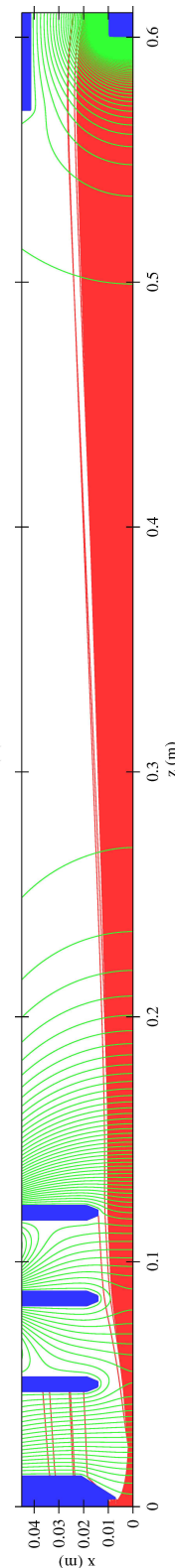

(c) $57.5^{\circ}$

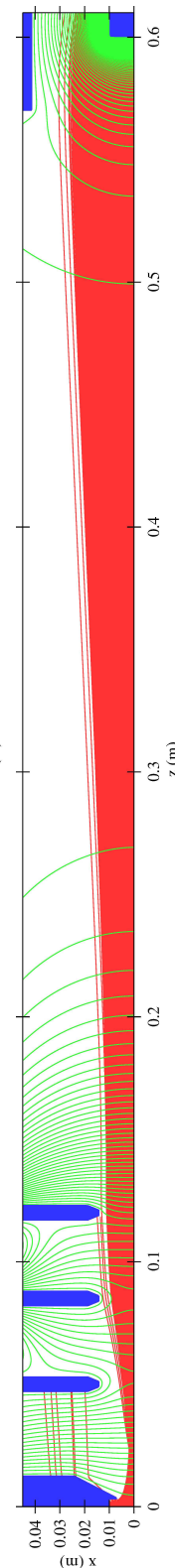

(d) $62.5^{\circ}$

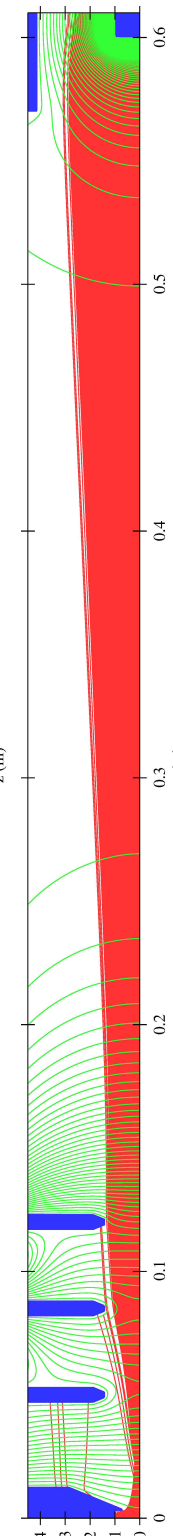

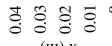

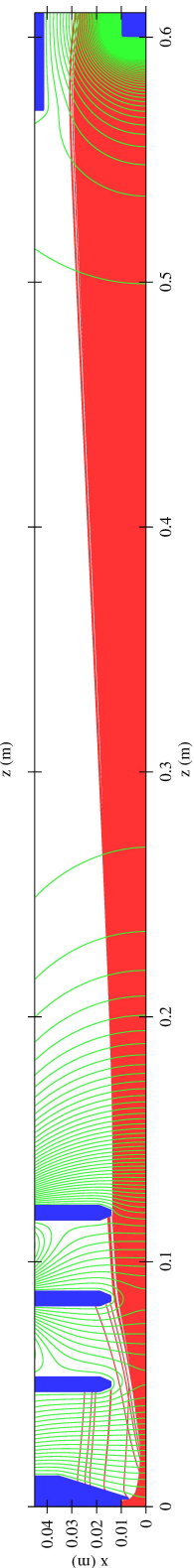

(f) $72.5^{\circ}$

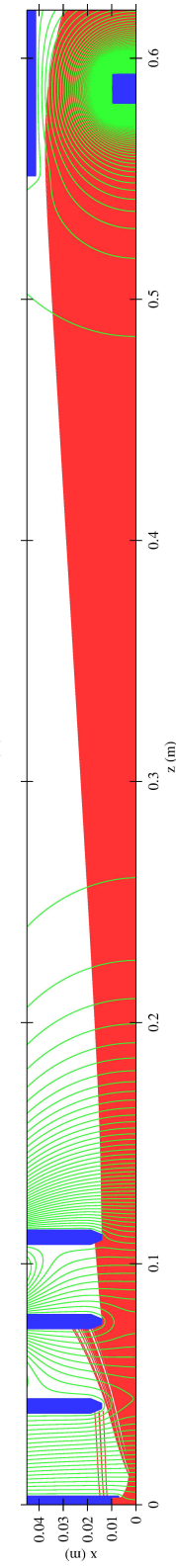

(g) $90^{\circ}$

Figure 24: Full beamline simulations with no Einzel lens, one extraction electrode, and Einzel lenses with different extraction angles to show trajectories. The $x$-axis is the distance to the center of the beam and the $z$-axis is the direction of travel for the ion beam. The system is nearly axially symmetric about $z$ 


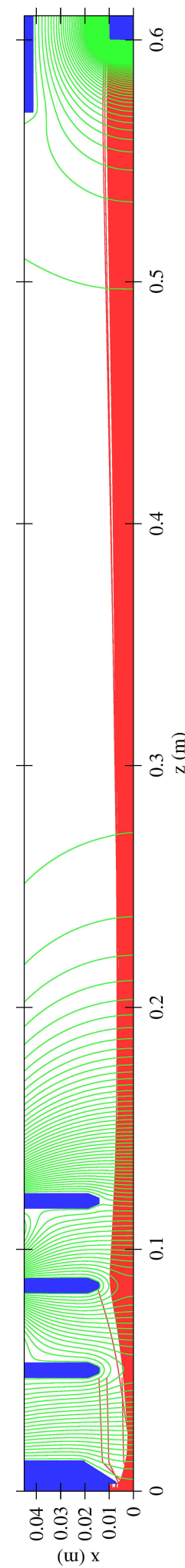
(a) $57.5^{\circ}$
(b) $62.5^{\circ}$

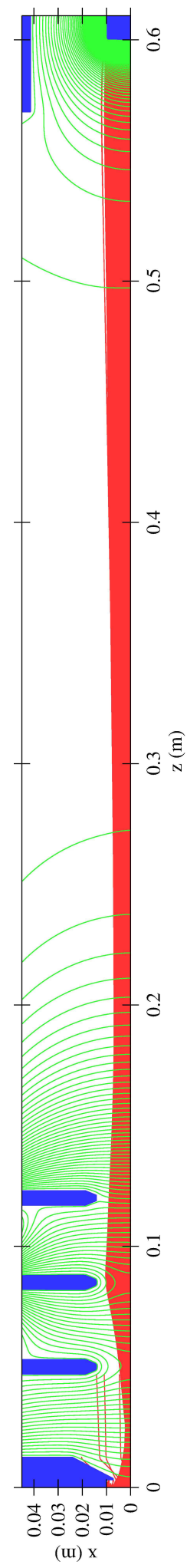

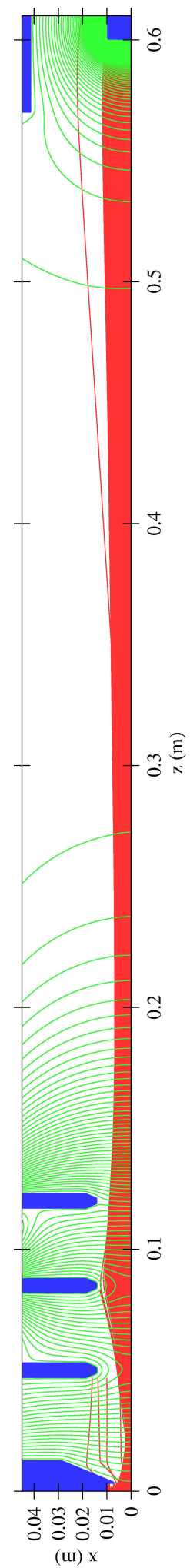

(c) $67.5^{\circ}$
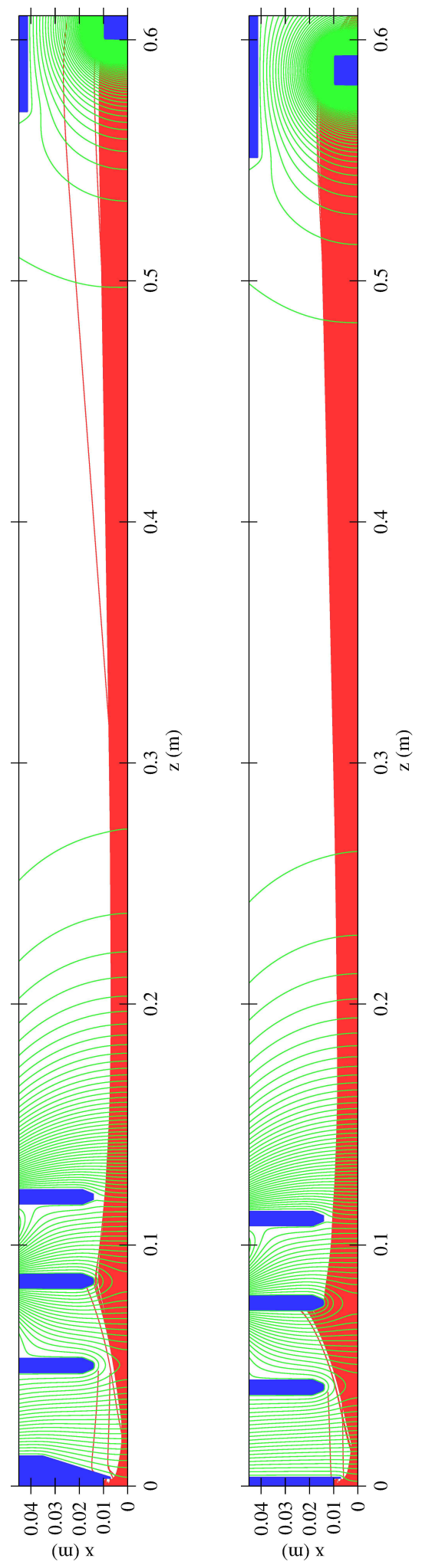

(d) $72.5^{\circ}$

(e) $90^{\circ}$

Figure 25: Full beamline simulations to show trajectories 


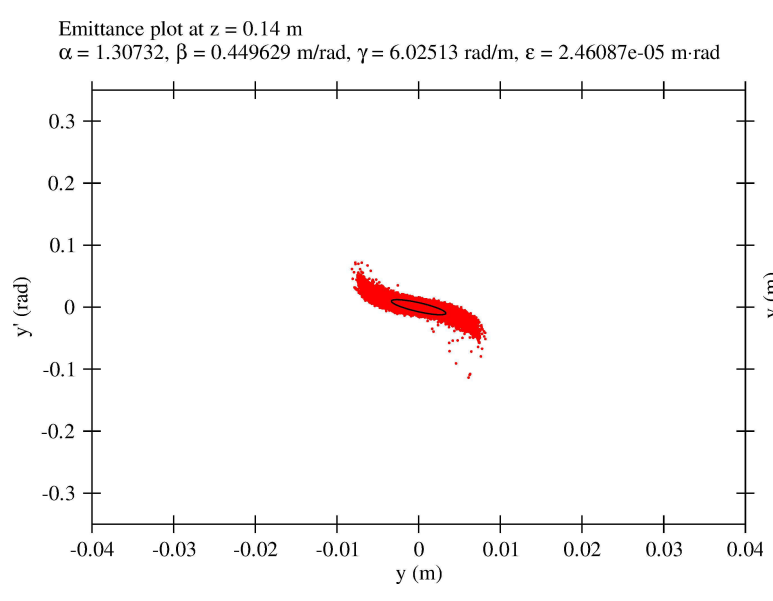

(a) Emittance Plot

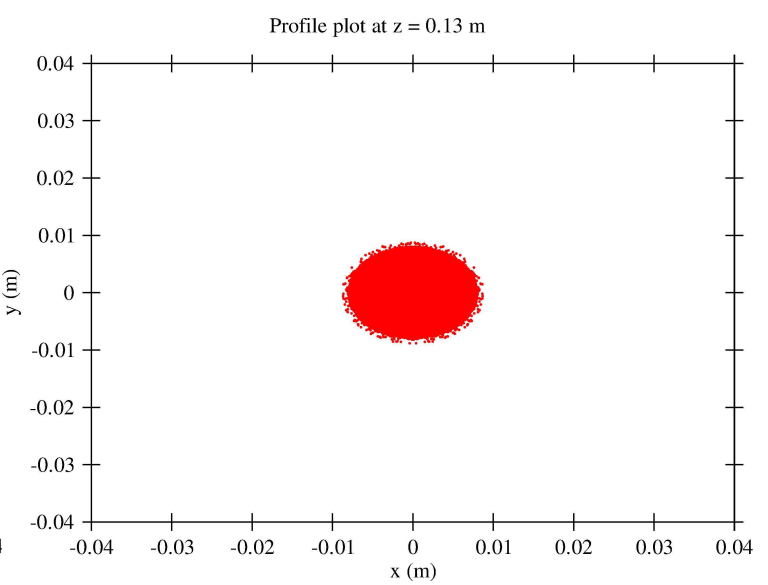

(b) X-Y Profile

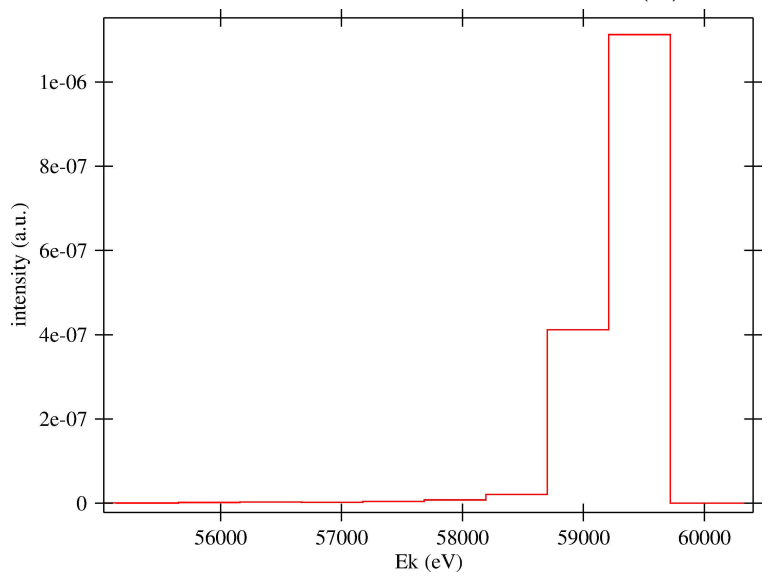

(c) Energy distribution just before target

Figure 26: Demonstration of beam characteristics after passing through the Einzel lens, $x$ and $y$ are distances to the center of the beam travelling along the $z$-axis. Emittance plot taken after the Einzel lens. 


\subsection{Final System Design Parameters}

The designed dimensions of the manufactured componentsare shown in Figure 27, and the separation of the electrodes, and their voltages are given in Tables 9 and 10 .

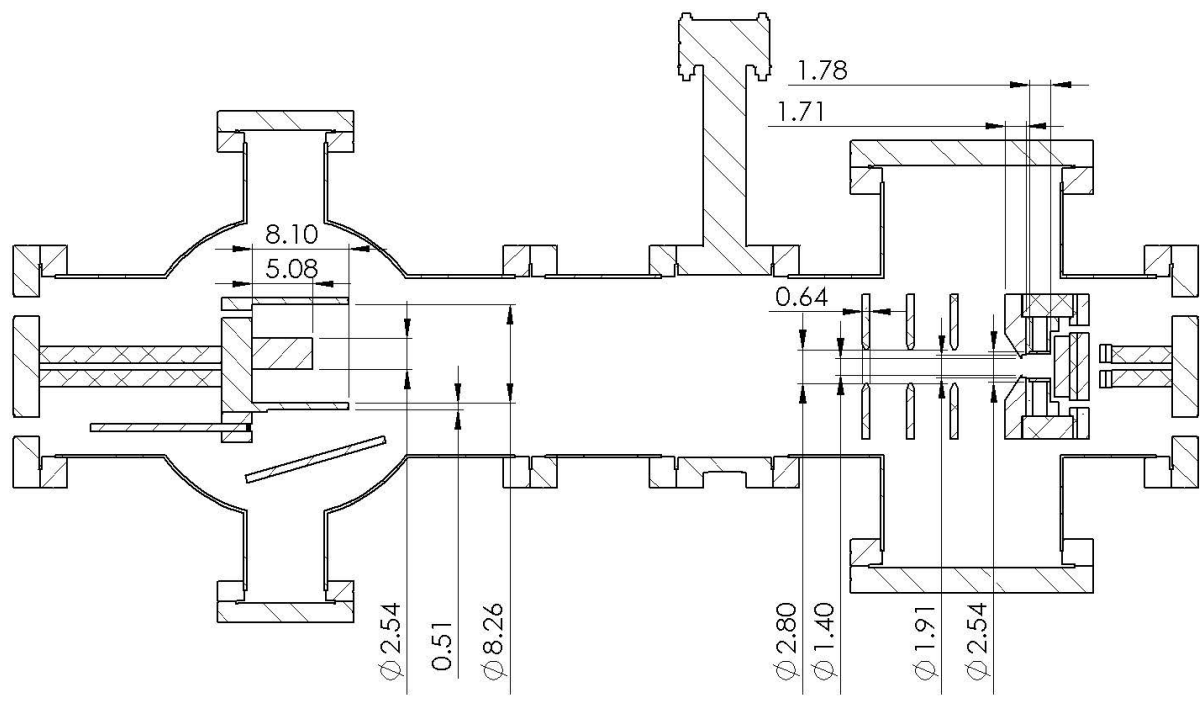

Figure 27: Top-down view of slice of beamline with major component dimensions. All values are in centimeters

Shown in Figure 27, is the PIG on the right-hand side, and the target on the left-hand side.

Table 9: Required separation of components as determined by the simulations

\begin{tabular}{c|c} 
Elements & Separation $(\mathrm{cm})$ \\
\hline Plasma electrode to first Einzel lens electrode & 5.0 \\
\hline Einzel electrode to Einzel electrode & 3.0 \\
\hline Last Einzel electrode to target & 50.0
\end{tabular}


Table 10: Electrode Potentials

\begin{tabular}{c|c} 
Electrode & Potential (V) \\
\hline Beamline Chamber & GND \\
\hline Plasma Electrode & $3 \times 10^{4}$ \\
\hline First Einzel Electrode & $2 \times 10^{4}$ \\
\hline Second Einzel Electrode & $2.8 \times 10^{4}$ \\
\hline Third Einzel Electrode & $2 \times 10^{4}$ \\
\hline Target Sub-chamber & GND \\
\hline Target & $-3 \times 10^{4}$
\end{tabular}




\section{Construction and Experimental Results}

\subsection{Construction of Vacuum System}

The first stage of construction was centered around the support frame for the system. This process, shown in Figure 28 was done using the Solidworks model as a guide. Having completed the frame, the chamber was then assembled and mounted

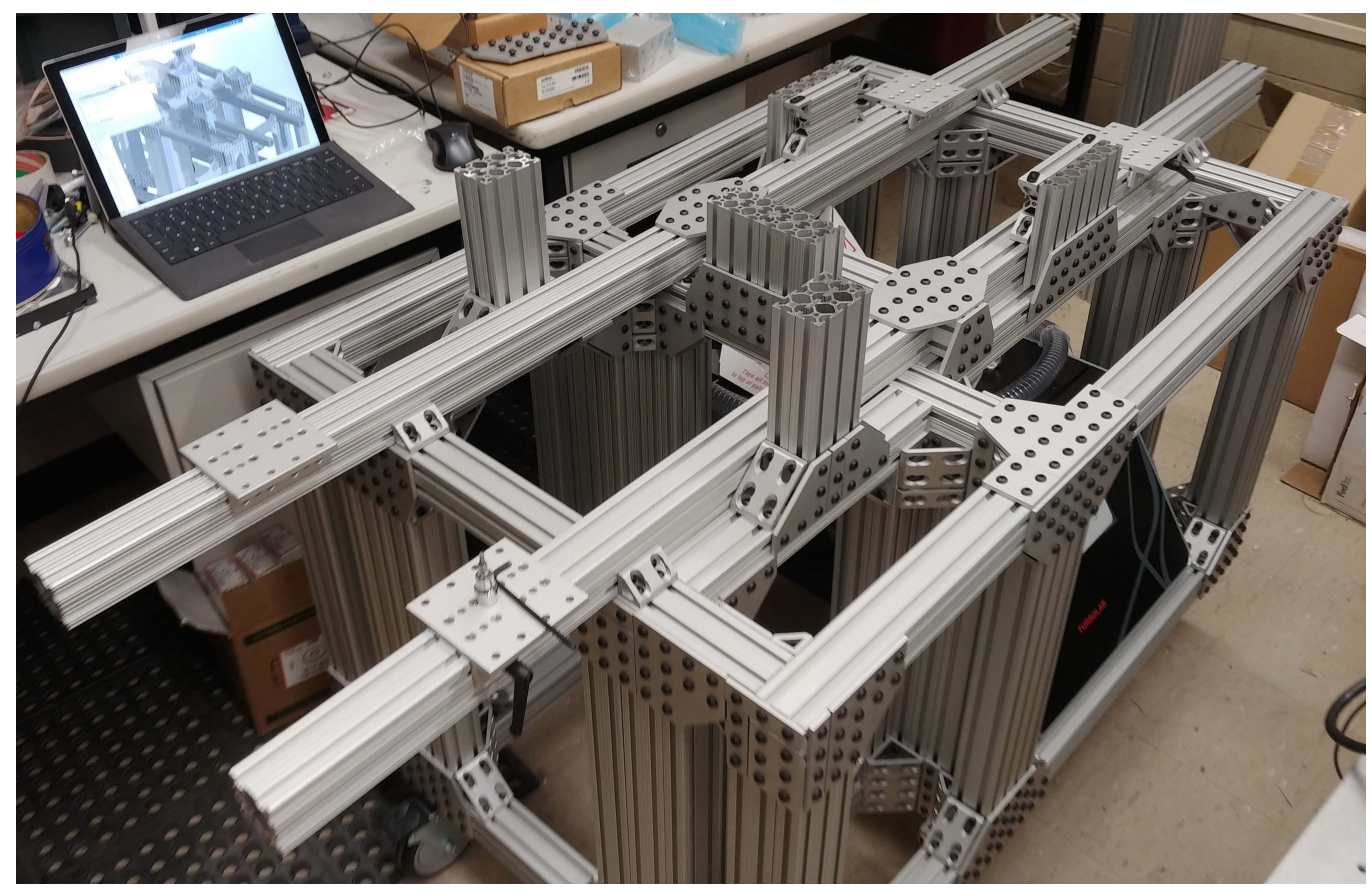

Figure 28: Construction of the frame

onto the frame. Due to the positioning of the gate valves and turbopump, it was concluded that the best approach would be to assemble the chamber vertically as seen in Figure 29. Once this was completed, the chamber had to be lifted and then lowered onto the frame using a ceiling-mounted monorail crane. This was necessary because of the chamber being entirely constructed of 304-SS and 316-SS, and having an odd shape that made it impossible to mount by hand. The chamber lowered into place can be seen in Figure 30 . 


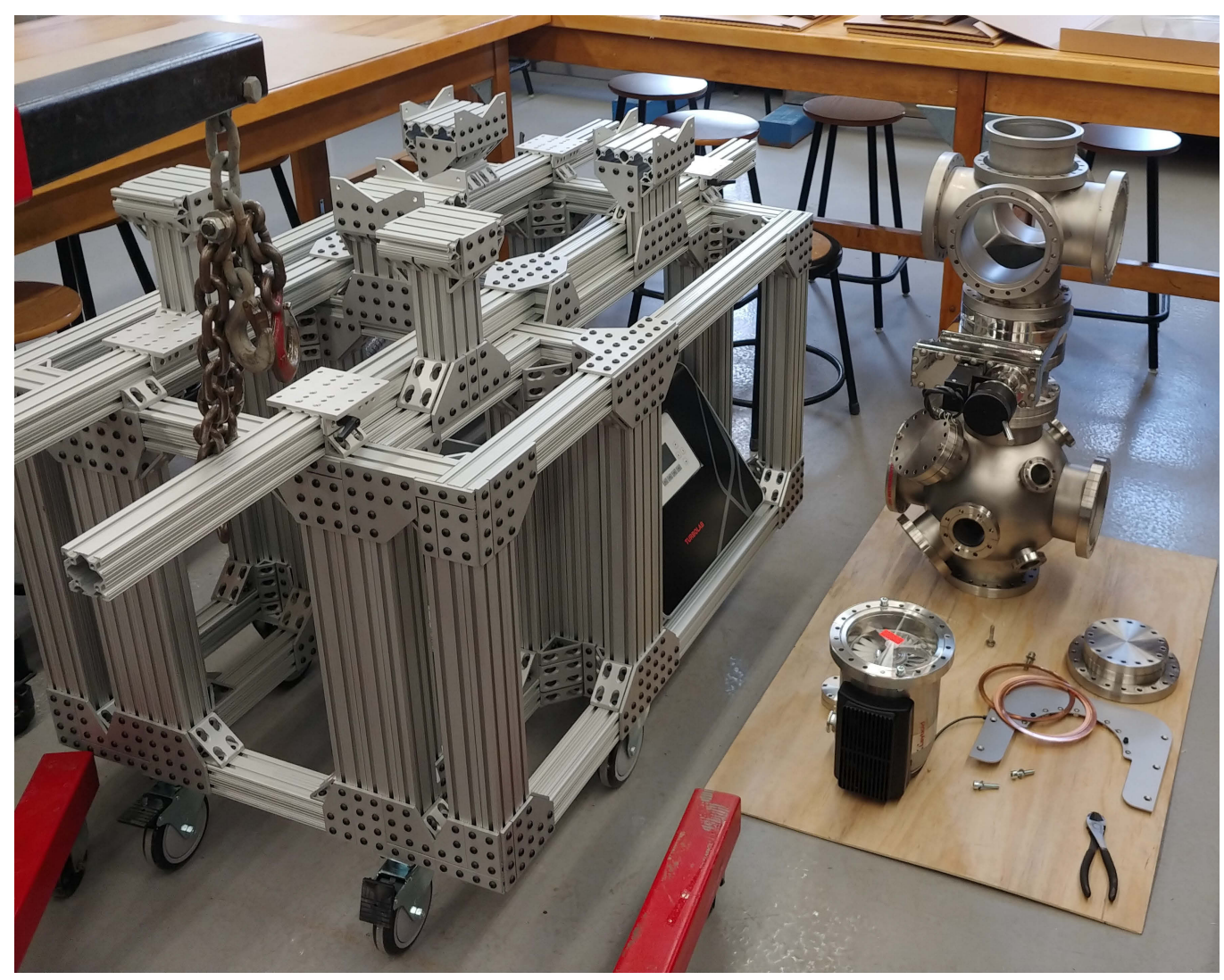

Figure 29: Assembling the chamber before lowering it into place 


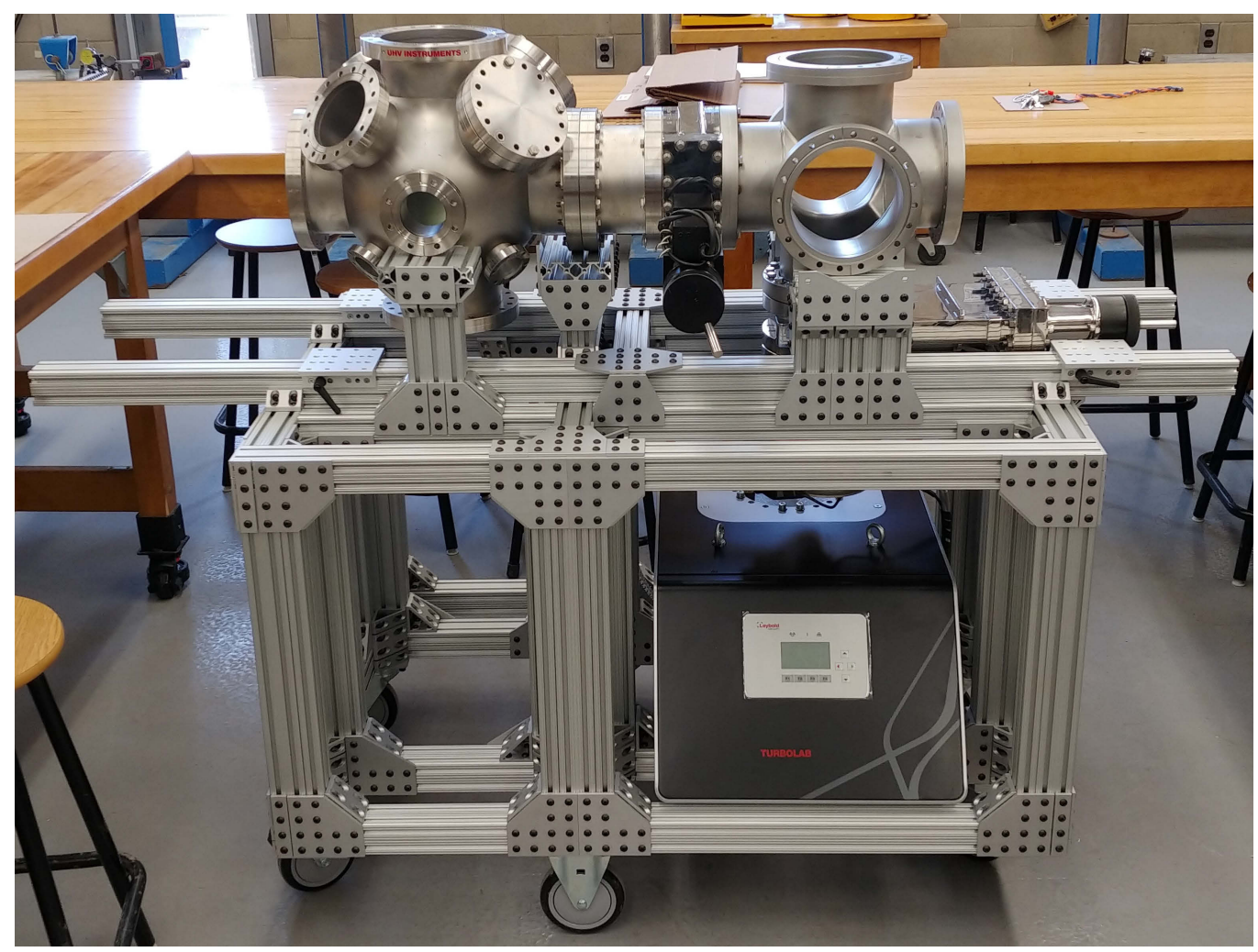

Figure 30: Chamber positioned on frame 


\subsection{Vacuum System Results}

Once the construction of the chamber had been completed, the remaining flanges were attached. The completed chamber is shown in Figure 31. An RGA was then

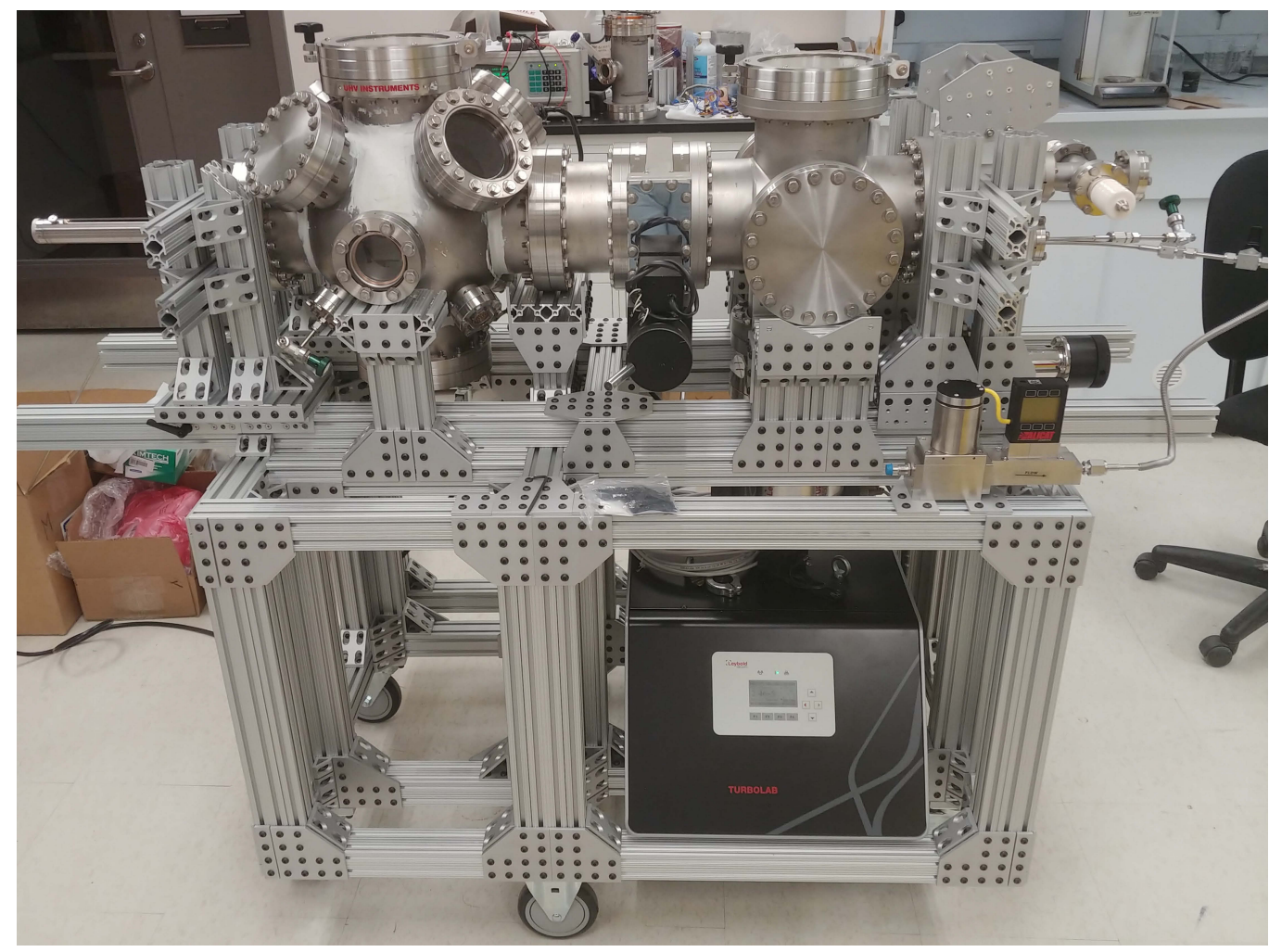

Figure 31: Completed vacuum chamber and pump, sealed for initial vacuum testing

attached to the system, the chamber was pumped down, and once the pressure had stabilized, the RGA was powered up and the background gas was analyzed. The RGA results after the first pump down are shown in Figure 32. As can be seen from the spectrum, the background gas of the chamber contained significant amounts of water, nitrogen, and oxygen as indicated by the peaks at mass channels 17, 18, 28, and 32 . This indicated that there may be a leak as the presence of such high quantities of oxygen and nitrogen, as well as the high ultimate pressure of $1 \times 10^{-5}$ Torr was not inline with the expected performance of the system. In order to confirm the leak, the chamber was left to pump down for two days, at which point the background gas was analyzed again. The second analysis, shown in Figure 33, revealed that the 


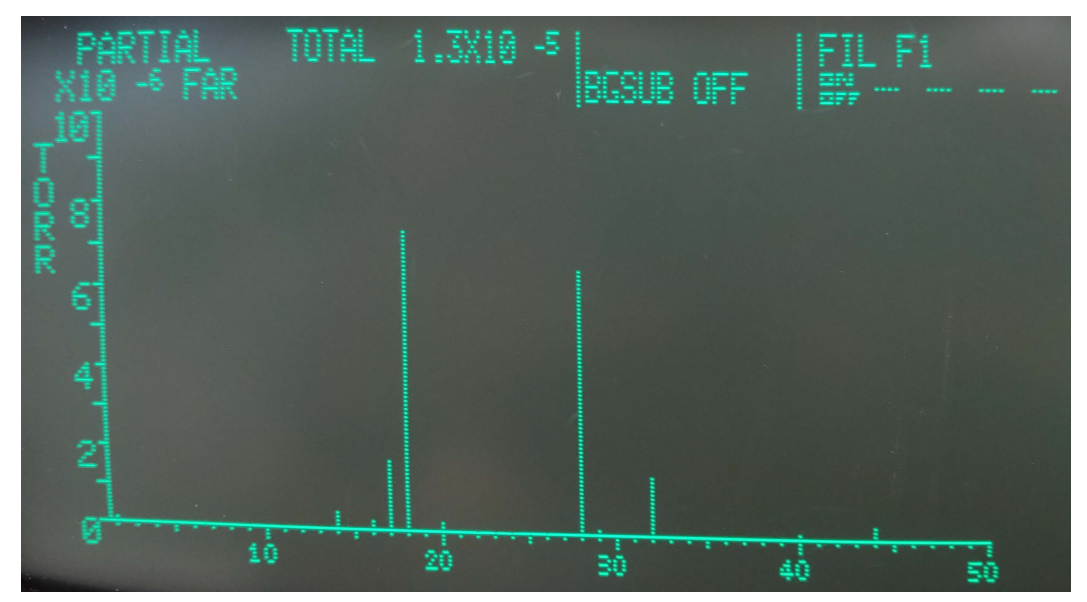

Figure 32: RGA results after the first pump down

water content had significantly reduced, but the nitrogen and oxygen had persisted, confirming the leak. In order to identify the source of the leak, helium leak checking done. The leak was found to be around the welds of all of the CF8 and CF4.5 flanges. It is suspected that this was a result of two compounding factors. Due to the chamber

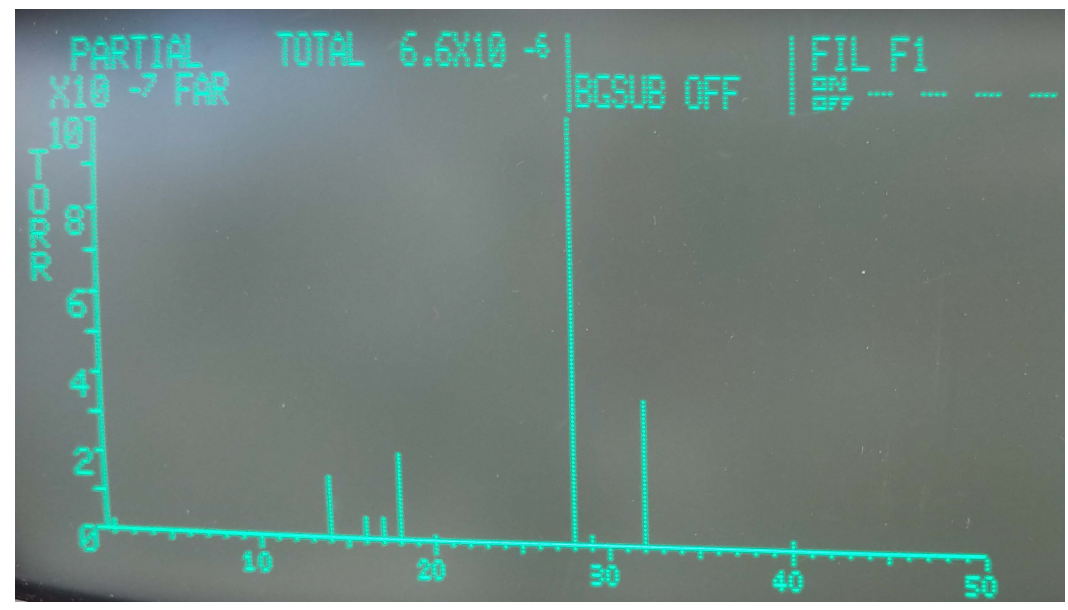

Figure 33: RGA results after a multi-day pump down

being formed of two hemispheres welded together, with the nipples then inserted and welded to the sphere only from within; and those six flanges being the most likely to bear weight or be struck during movement, these became fault lines for micro cracks to form. The six flanges were sealed by applying Torr-Seal, a specialized epoxy for use in vacuum chambers, around all the nipples, preventing any ingress. Once the 
epoxy had cured, the RGA was again used to analyze the residual gasses present in the system. The resultant spectrum is visible in Figure 34. This spectrum with

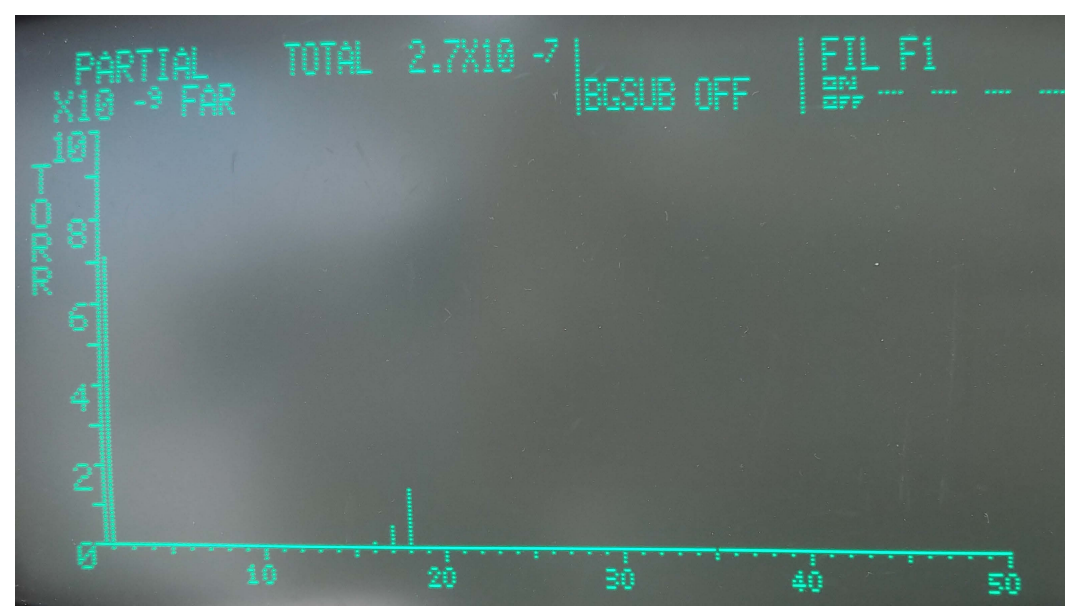

Figure 34: RGA results after successful sealing of all leaks

only minute traces of water, and detection of hydrogen (mass channel 2) indicated all leaks to atmosphere had been sealed, and only virtual leaks from the water monolayer remained. As confirmation, the ultimate pressure of the chamber after two days of pump down was $<5 \times 10^{-8}$ Torr. The readout of the vacuum gauges can be seen in Figure 35. The pump was then shut down and the system pressure was monitored as

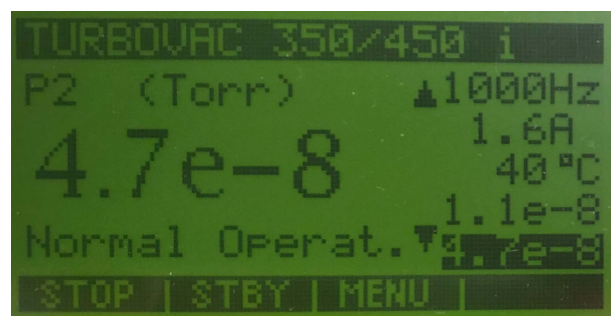

Figure 35: Pressure of the chamber 24 hours after application of epoxy to leak

it rose. The resultant RGA spectrum, seen in Figure 36 confirmed the elimination of any leak with a spectrum containing carbon monoxide, as indicated by the significant peak at 28 and the minor peaks at 12 and 13; carbon dioxide, as indicated by the peaks at 44, 28, and 12; hydrogen and water. This water could be eliminated by baking the system, however due to the very low concentration of water (a partial 
pressure of $<2 \times 10^{-9}$ Torr), it was concluded that it was unnecessary to remove such minimal quantities as the system was expected to operate with a background gas pressure of $>1 \times 10^{-6}$, that resulted from the inflow of gas for the plasma formed in the PIG.

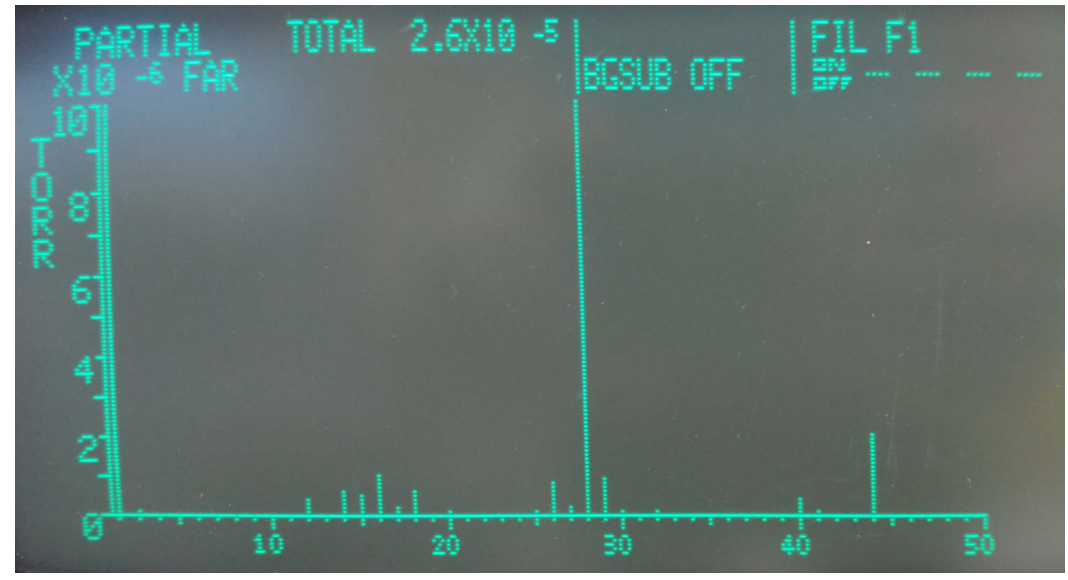

Figure 36: RGA results under static vacuum

\subsection{Construction of Internal Components}

The Construction of the internal components involved the manufacturing of the PIG, the Einzel lens electrodes, and the target sub-chamber. This was done inhouse by the author. The PIG was made by turning and boring $13 \mathrm{~cm}$ cylindrical stock aluminum and 304-SS on a lathe. 304-SS was the material of choice for the plasma electrode because of the superior hardness and resistance to ablation by the ion beam that it would have over aluminum. The remaining components were made of aluminum for increased ease of manufacturing and reduced weight of the system, which simplified mounting. Additionally, by constructing the PIG in sections, it allowed for the easy swapping of components to accommodate for wear of minor design changes; a feature especially useful in the case of the plasma electrode where component lifetimes are known to be short. The completed components are seen in Figure 37. 


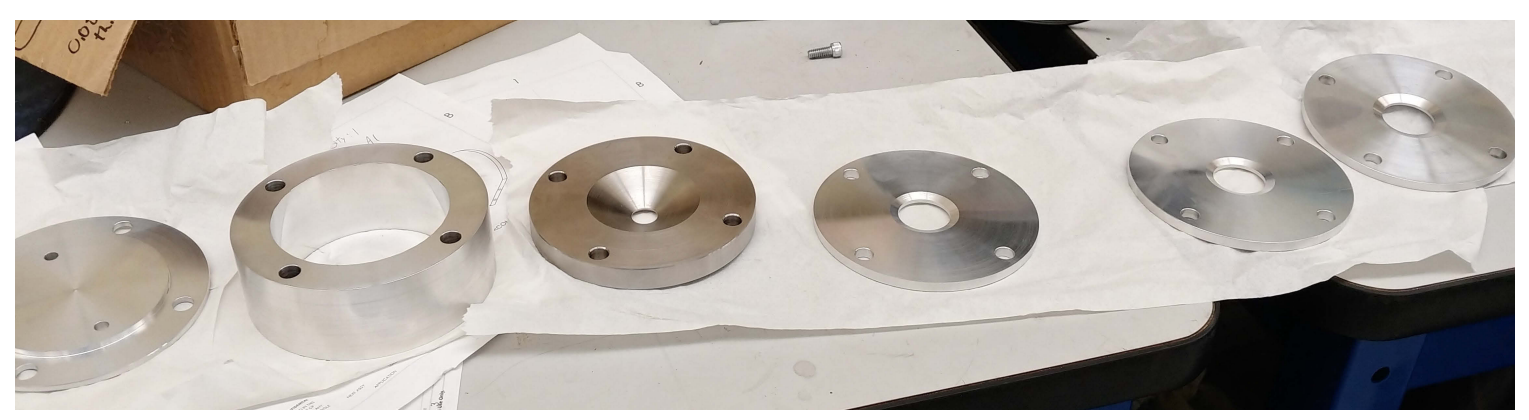

Figure 37: Manufactured Components for PIG and Einzel Lens

Due to the more complex shape of the target sub-chamber, it was made of a solid aluminum $10 \mathrm{cmx} 11 \mathrm{~cm}$ block using a Computer Numerically Controlled (CNC) mill. As before, aluminum was selected for ease of manufacture and lower weight. The CNC milled component is shown in Figure 38.

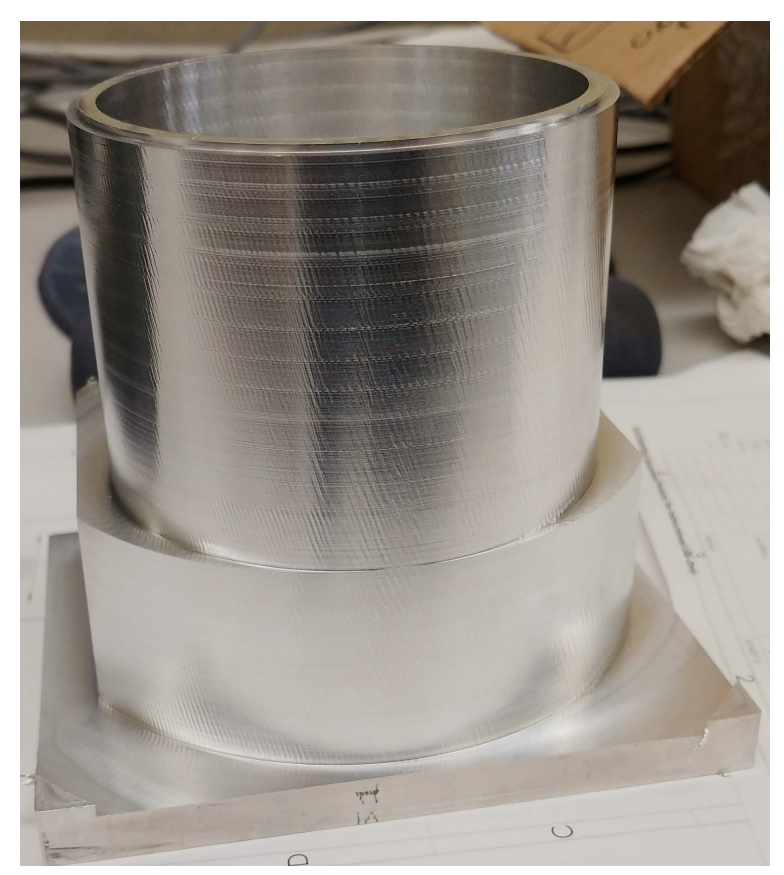

Figure 38: CNC Milled target sub-chamber

Having manufactured the necessary components, they were then assembled into the final structure. The assembly of the PIG and its associated Einzel lens is shown in Figure 39. Visible in Figure 39a, is the PIG anode surrounded by a ring magnet and supported by electrically isolating ceramic discs. The wiring and copper tape guarantee good electrical contact from supply to the anode. 


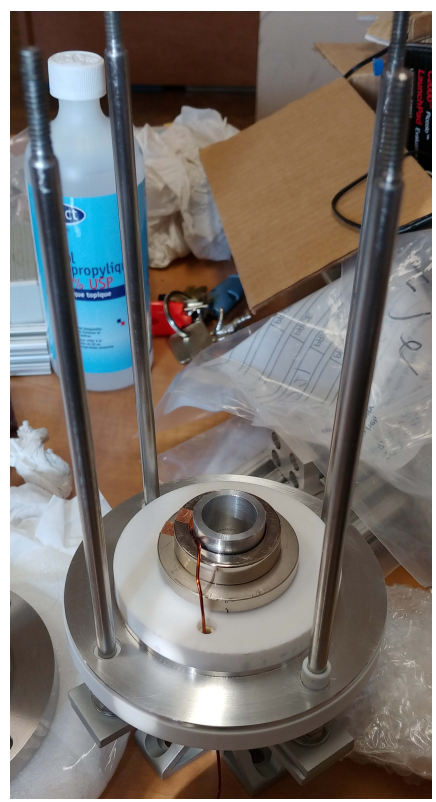

(a) PIG Anode

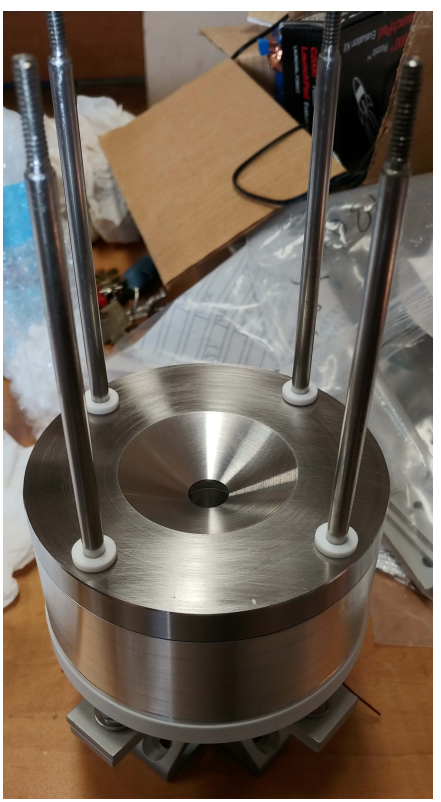

(b) Assembled PIG

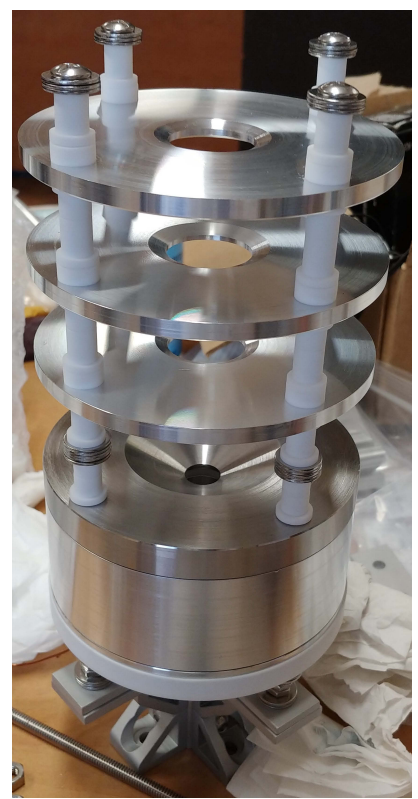

(c) Assembled PIG with Einzel lens

Figure 39: Assembly of PIG and Einzel Lens

The components were finally mounted and electrically connected through the vacuum chamber as described by the electrical schematic for the system shown in Figure 40. The mounting of the target sub-chamber is seen in Figure 41. The mounting of the PIG with the Einzel lens is shown in Figure 42, and finally the electrical supply system is shown in Figure 43. The target sub-chamber and target were electrically isolated to investigate the proportion of current reaching each. 


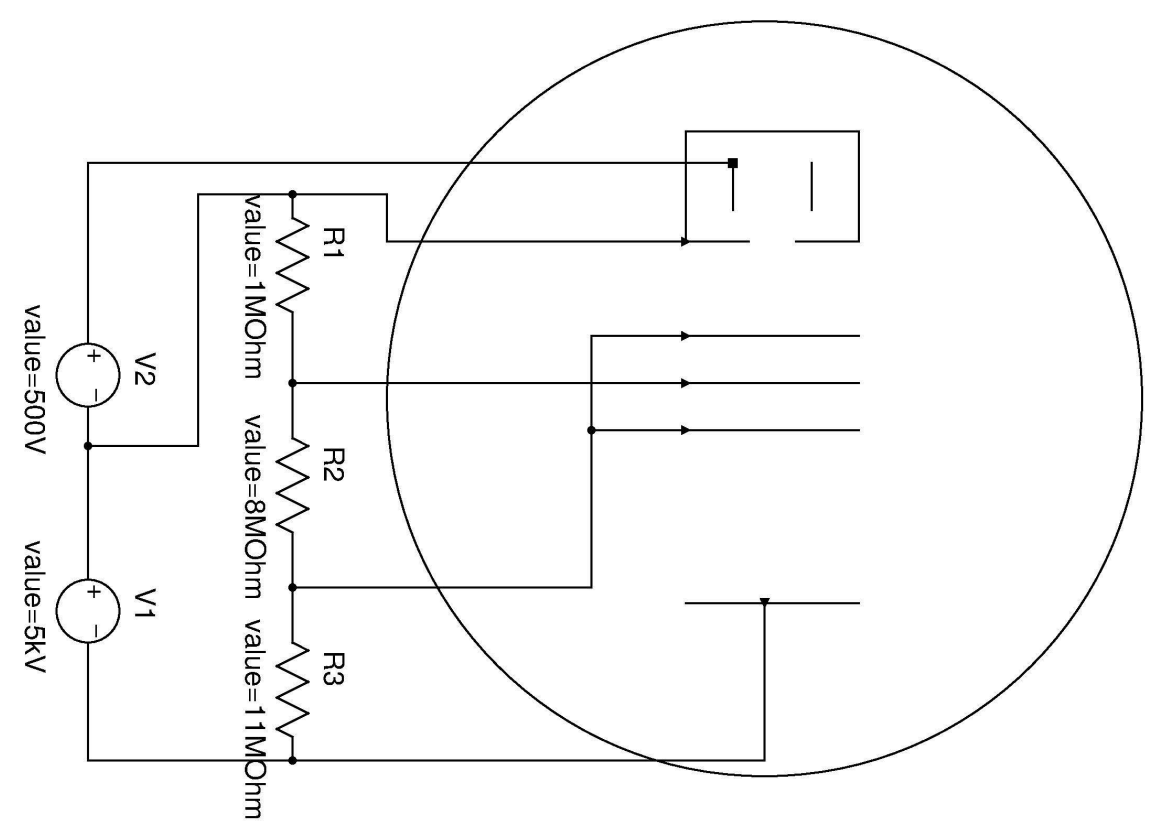

Figure 40: Electrical schematic of system

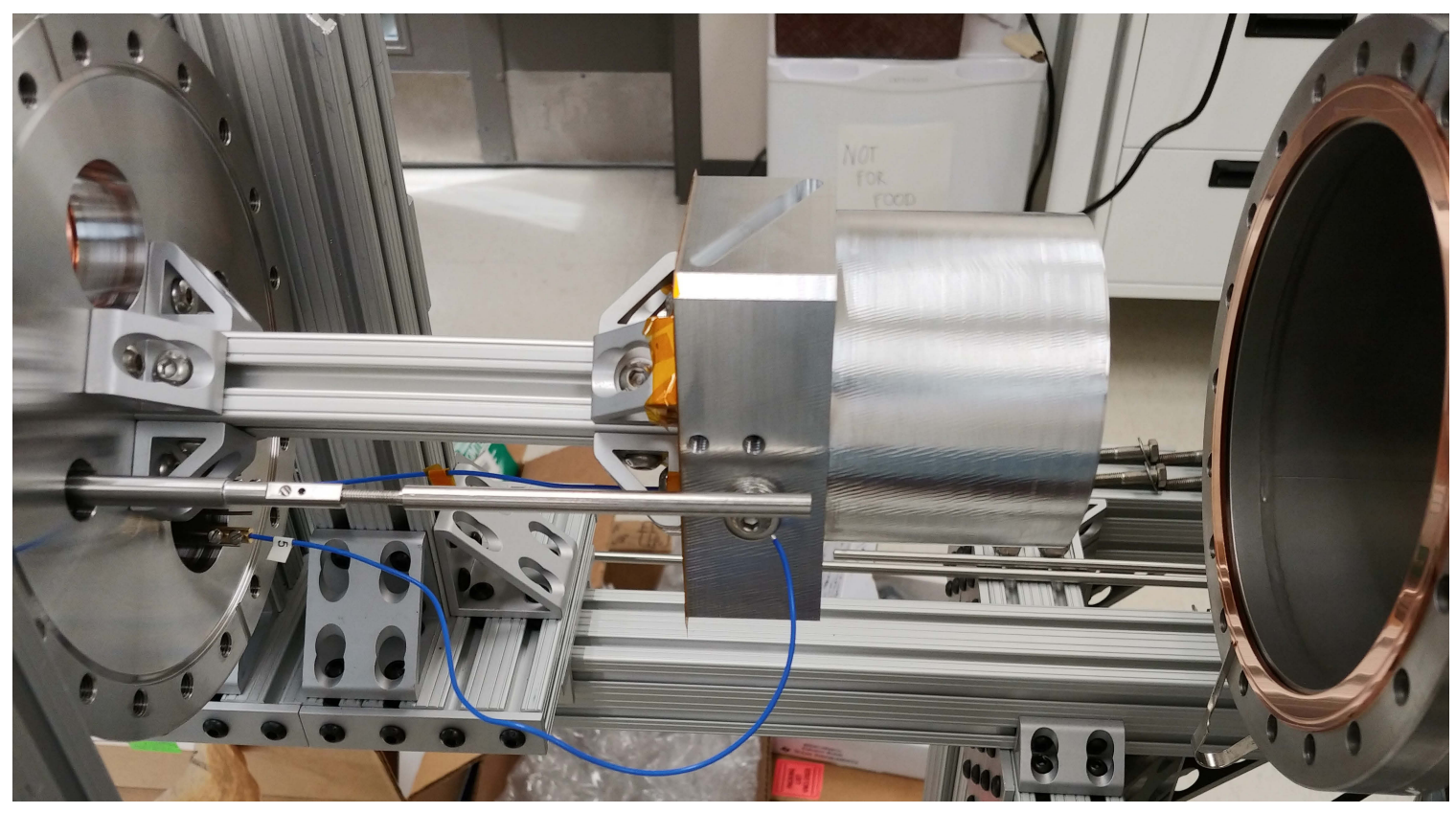

Figure 41: Mounted and electrically connected target sub-chamber 


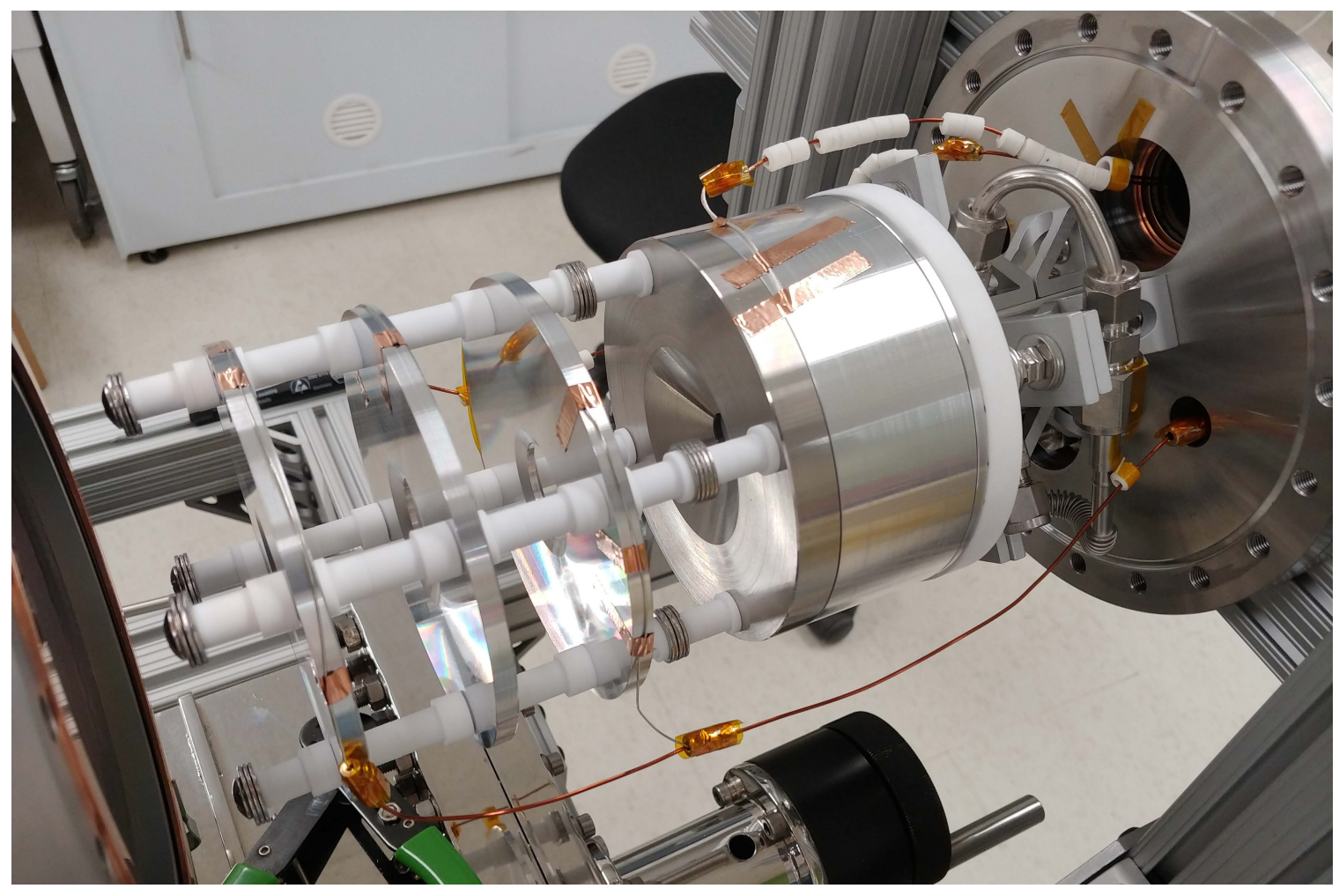

Figure 42: Mounted, electrically connected, and gas connected PIG with Einzel lens 


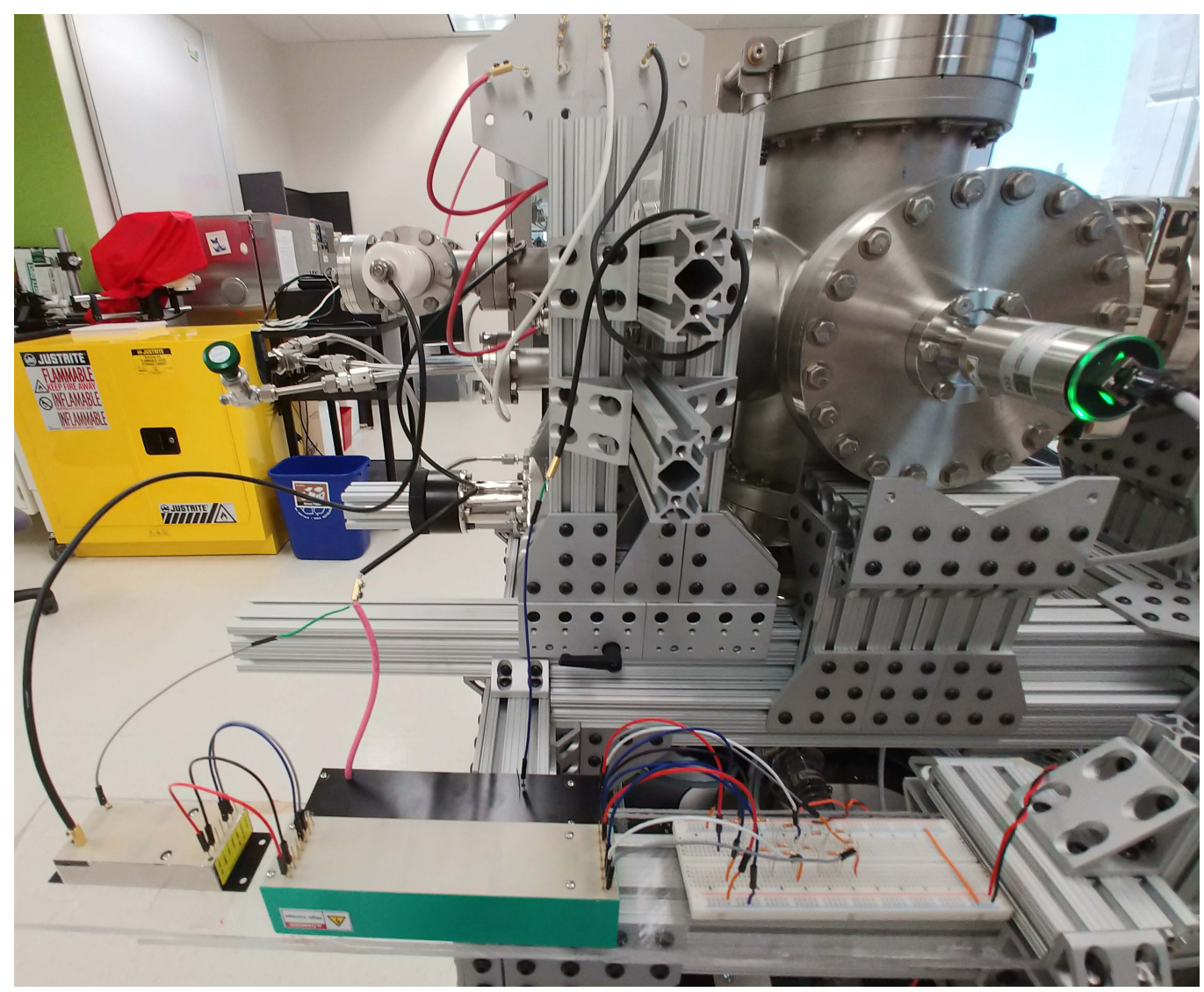

Figure 43: External electrical connection for system 


\subsection{Plasma}

\subsubsection{Nitrogen Plasma}

To verify the plasma source operated as intended, the acceleration potential was limited to $5 \mathrm{kV}$ and a potential different of $500 \mathrm{~V}$ between the PIG anode and cathode was applied. First plasma was achieved using a nitrogen backing gas. Plasma was struck around $8 \times 10^{-2}$ Torr and would persist down to $7 \times 10^{-3}$ Torr. An image of this plasma is seen in Figure 44. As the pressure was decreased by opening the gate valve to the turbopump, the decrease in pressure allowed for ions to reach the target and for current to be measured. A maximum current of $2.9 \mu \mathrm{A}$ was measured on both the target and the target sub-chamber. It was observed that a distinct beam would form, travelling along the path formed by the Einzel lens, but the beam would be unstable and would flicker. An optical spectrum was collected and is shown in Figure 45. As described by Bayram and Freamat [41] and Wagatsum and Hirokawa [42], the expected optical spectrum of molecular nitrogen $\left(\mathrm{N}_{2}\right)$ has 3 distinct peaks of decreasing intensity at approximately $390 \mathrm{~nm}, 380 \mathrm{~nm}$, and $375 \mathrm{~nm}$. This is followed by two more distinct peaks of higher and increasing intensity at $355 \mathrm{~nm}$ and $335 \mathrm{~nm}$. All of these peaks are clearly visible, indicating an $\mathrm{N}_{2}$ plasma.

To verify the composition of the fill gas, a spectrum was collected using the RGA. The spectrum is shown in Figure 46. The characteristic intense peak at channel 28 of nitrogen is clearly visible, but so too are the peaks of water at 18, and oxygen at 32, indicating that the nitrogen inlet was leaking, or had not been fully purged of oxygen. The water was persistent throughout all tests as the surfaces of most components were still off-gassing. 


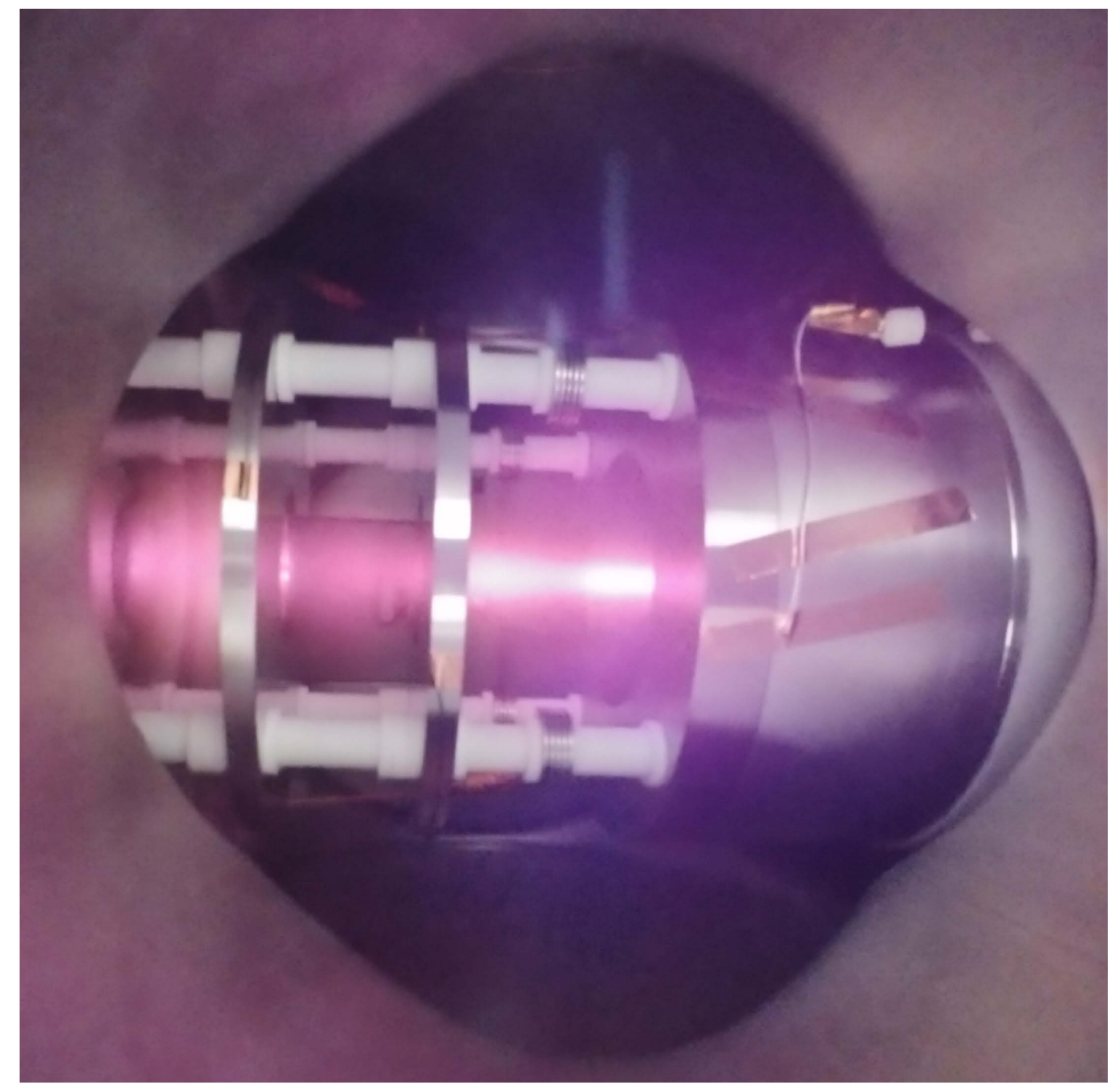

Figure 44: Image of nitrogen plasma and extracted beam 


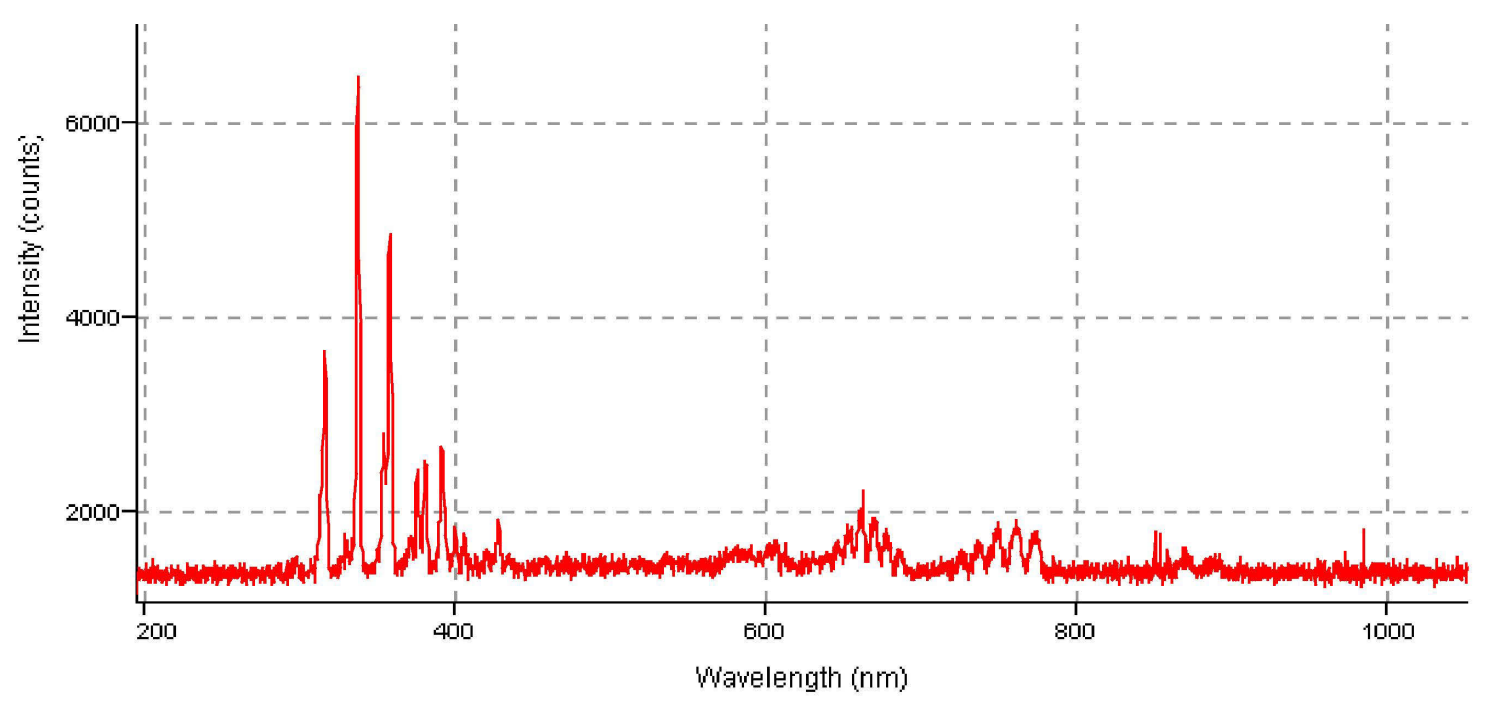

Figure 45: Optical Spectrum of nitrogen plasma

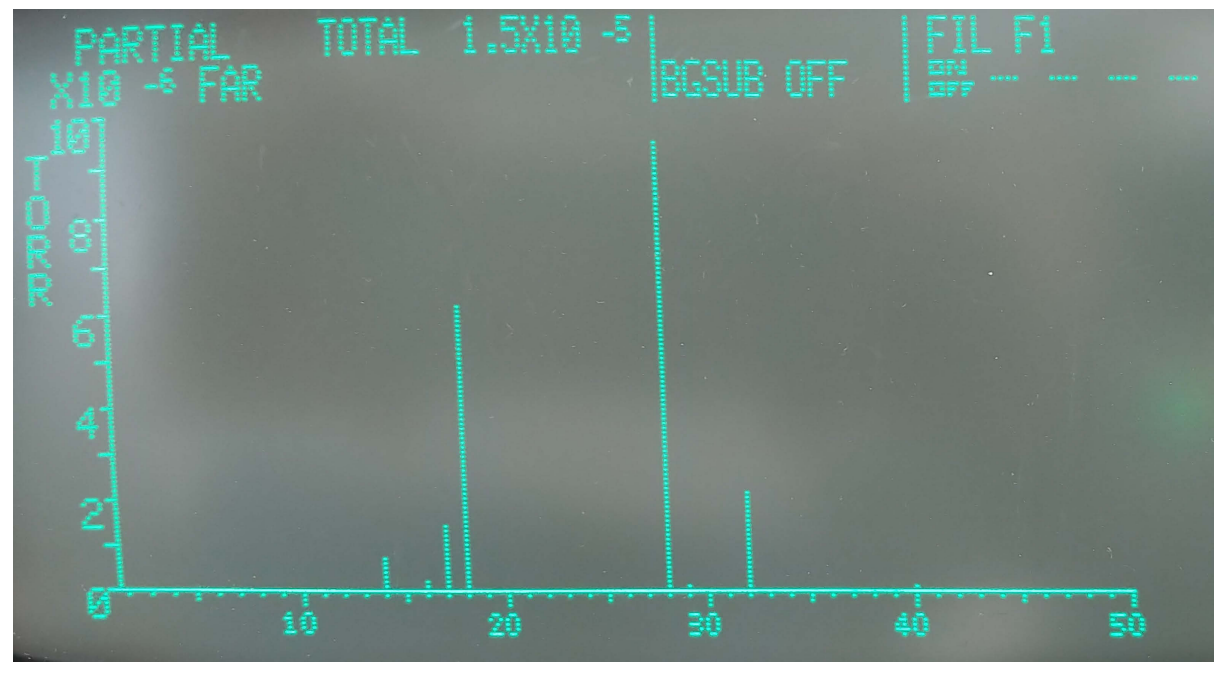

Figure 46: RGA spectrum for nitrogen plasma trials 


\subsubsection{Argon Plasma}

A second plasma was struck using an argon fill gas. The plasma created is shown in Figure 47. The pressure for gas formation was in the same range as for the nitrogen plasma, between $8 \times 10^{-2}$ Torr and $7 \times 10^{-3}$ Torr. No current was detected at the target or the sub-chamber. It was observed that as the beam would focus, at the most focused a crackling would come from the resistor array and the plasma would extinguish. The optical spectrum collected from the plasma is shown in Figure 48.

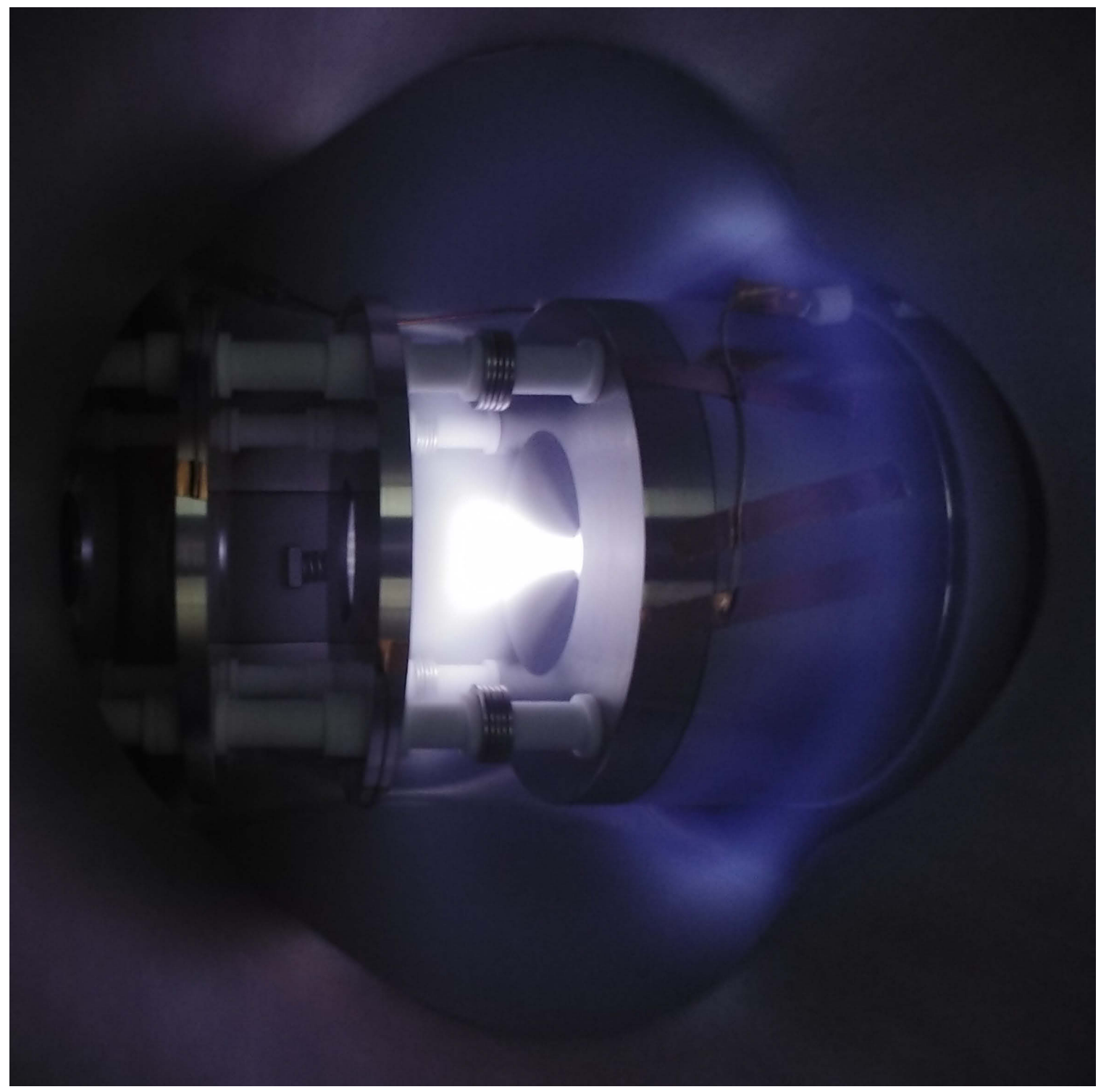

Figure 47: Image of argon plasma and extracted ions 
The check on the background gas is shown in the RGA spectrum shown in Figure 49.

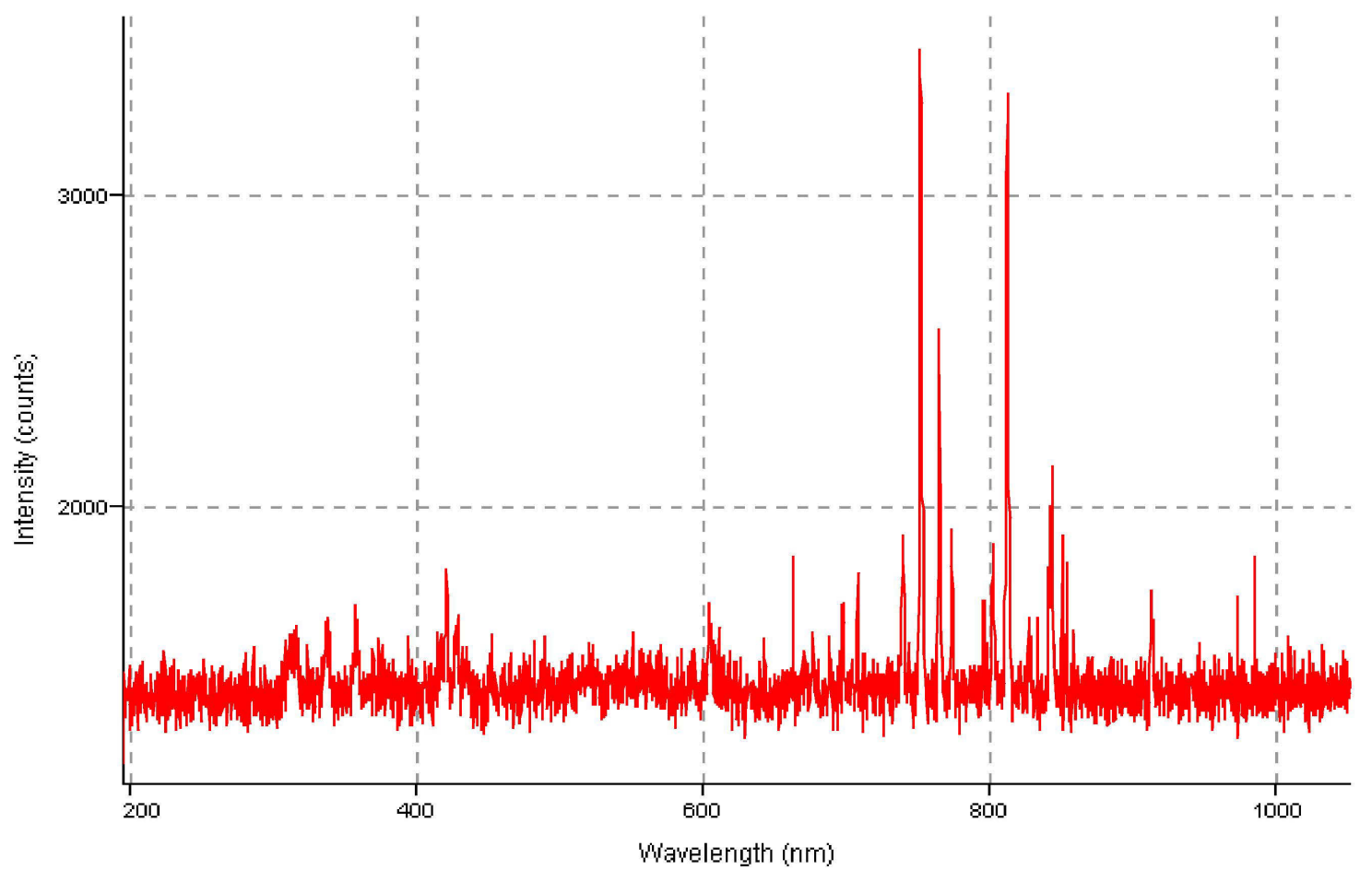

Figure 48: Optical spectrum of argon plasma

The distinct peaks at 40 and 20 clearly demonstrates the presence of argon. 


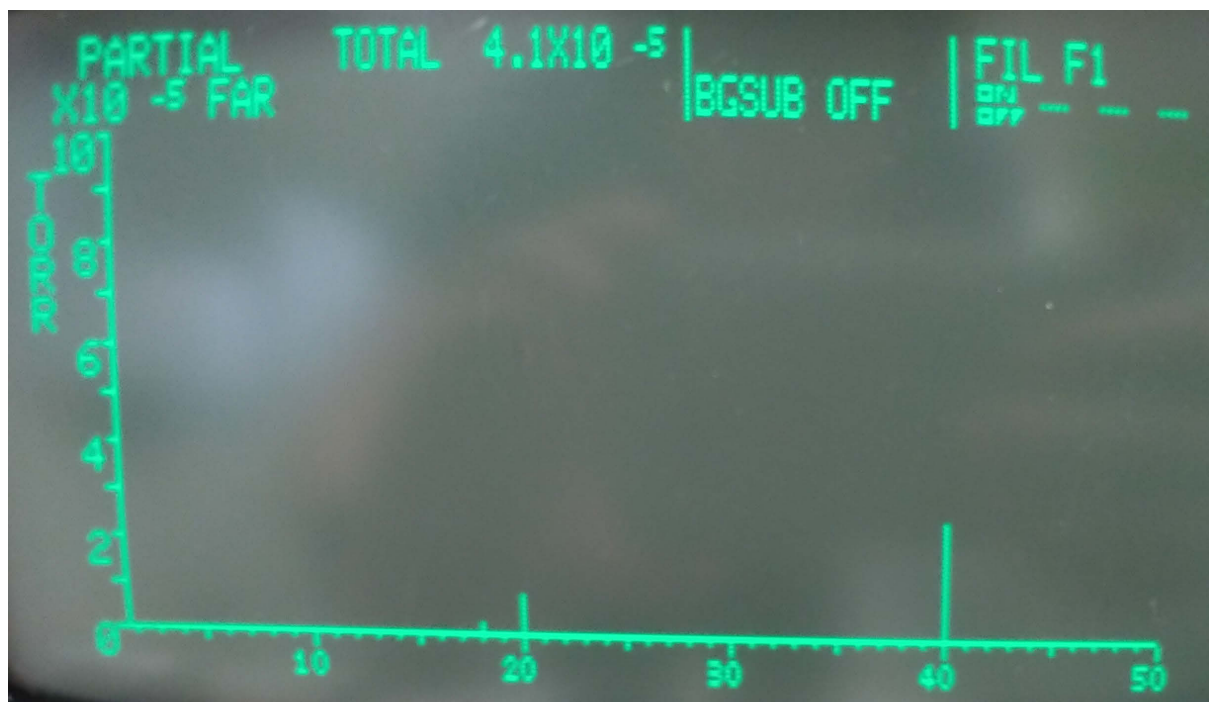

Figure 49: RGA spectrum for argon plasma trials 


\section{Discussion}

While the achieved plasma is an important first step in the process development of the system, three primary issues were observed that will or could present issues in the transition to higher operating voltages and formation of a clean beam as designed for. These are the high pressure for igniting and maintaining the plasma, the arcing through the insulating supports on the resistor array, and sparking within the vacuum chamber.

\subsection{Pressure Management}

The pressure range at which the plasma was found to exist $\left(8 \times 10^{-2}\right.$ Torr to $7 \times 10^{-3}$ Torr) is greater than the required $1.3 \times 10^{-4}$ Torr to provide the needed Mean Free Path (MFP). These pressures were through a combination of varying flow rate, and varying the opening to the turbopump by way of the gate valve between the source chamber and it.

The objective was to have a high enough pressure within the Penning Ion Generator (PIG) source to strike a plasma, but to still maintain the low pressure needed for the path length of the ions to reach the target. This was difficult to attain as it is suspected there was significantly more leakage from the PIG than anticipated. This may be due to either the threading on the support rods being slightly angled, reducing the tightness of the seal, or more simply, that the mated components making up the PIG cannot seal sufficiently to have a low enough leakage rate to manage the pressure within the chamber. Opening the gate valve and increasing the flow rate proved ineffective as this appeared to result in the plasma being physically forced out of the PIG. 


\subsection{Resistor Array Arcing}

A common occurrence in the initially formation of the plasmas was a cone of plasma between the plasma electrode and the first Einzel lens electrode. As the pressure of the system was gradually reduced, this cone would narrow until a focused beam could be observed. Shortly after formation, an audible crackling could be heard originating from the resistor array, and the plasma would extinguish. It is suspected that this is a result of the increase in the voltage between the first Einzel lens electrode and the plasma electrode, which are connected via the resistor array. When the plasma starts to focus, the increase in potential could have caused the short to ground across the resistor array support. When the arc occurs this would cause a sudden loss of current to the plasma, and it would be extinguished.

\subsection{Vacuum Arcing}

During the operation of the system with a plasma present, occasional sparks could be observed eliminating from either the surface of the chamber or the surface of the metallic spacers used for positioning the Einzel lens components. It was suspected that this was simply a discharge of built up charge from the plasma and was unlikely to be an issue as the voltage was ramped up. Particularly as the pressure is brought down to its target operating range; however, adding a charge bleed component would eliminate this issue altogether. 


\section{Conclusions}

A vacuum chamber with a modular support system and a Penning Ion Generator (PIG) plasma ion source with Einzel focusing lens were designed, manufactured, and tested. The vacuum chamber was capable of attaining vacuum levels of $4 \times 10^{-8}$ Torr, and plasmas of both argon and molecular nitrogen were formed. The system was found to be capable of generating a maximum beam current of $2.9 \mu \mathrm{A}$ at an acceleration voltage of $5 \mathrm{kV}$.

Research and development of new power sources are, societally speaking, of paramount importance; and fusion represents one of the best possible future energy sources. This test bed and PIG plasma ion source provide the foundation for investigation of target materials, acceleration techniques, and projectile types and will be able to grow and evolve to meet the changing needs of the research, advancing understanding of fusion under a variety of conditions.

\subsection{Recommendations and Future Work}

\subsubsection{Chamber}

For proper detection of successful fusion events, a number of detectors are required. As many of the fusion processes of interest produce $\alpha$-particles, a first detector that would be required would be a pin diode array for energy discriminating $\alpha$-particle

detection. As $\alpha$-particles are stopped very easily by very little material, an array of pin diodes, some blocked by thin layers of metal, and other exposed would allow for the recording of coincidence and anti-coincidence spectra that would allow for discrimination of ejected fragments. Additionally a more integrated control system and data collection device that centralizes the control and monitoring of the system would be beneficial to the controlled operation of the test bed. Lastly, achieving fusion will require increased voltage, which as demonstrated by the arcing observed 
at the resistor array, will require the electrical systems to be revised. Redesigning the electrical systems to use supports made of Polytetraflouroethylene (PTFE) should allow for much higher acceleration potentials, enabling fusion to occur. Once these changes have been made and, an investigation into target material, target design, feed gasses, and projectile type and can be undertaken to further improve understanding of fusion. This could further be used to create a neutron source for investigation of plasma wall material choices for conventional forms of fusion reactors.

\subsubsection{Simulation}

In order to better predict and compare reactions, it would be beneficial to couple Geant4 into the beamline simulation suite. This would provide information of the bulk interaction of the ion beam with the target and the interactions of the fragments with the detectors.

Another important step would be to modify IBSIMU to have more automated resolution management. As it operates currently, it is necessary to manually identify the minimum acceptable resolution and apply this to the entire simulation section. While splitting up the simulation into subsection alleviates some of the issues around this, multi-resolution simulations would additionally accelerate the simulation time dramatically. Furthermore, improving parallelization automation to include memory usage prediction and management of which simulation components to run when would greatly reduce the burden on the designer when investigating many designs.

\subsubsection{Penning Ion Generator}

Improvements to the PIG that would be worth investigating include the benefits of a Halbach array as the source of the magnetic field as it could potentially allow for miniaturization of the source and improved magnetic field characteristics. 
Additionally as one of the downsides of operating a PIG source is the metal fragments that are alleviated by use of a hot cathode, it would be worth investigating the potential of a Spindt array or Field Emission (FE) array for improved efficiency and miniaturization. Miniaturization of the proton source and improved gas sealing. The extraction characteristics of the PIG could be improved through the addition of an intermediate extraction electrode between the plasma electrode and the first Einzel lens electrode. Doing this would allow for finer control of the plasma meniscus and the current density where the beam becomes current limited. This would also provide the Einzel lens with greater range of operating voltages and would allow it to perform well up to much higher acceleration potentials. Finally, improvements to sealing and investigation of PIG redesigns to improve gas flow characteristics and maintenance of a higher internal pressure would be beneficial to the behaviour of the system. 


\section{References}

[1] T. F. Stocker, D. Qin, G. K. Plattner, M. Tignor, S. K. Allen, J. Boschung, A. Nauels, Y. Xia, V. Bex, and P. M. Midgley, Climate Change 2013, The Physical Science Basis, Contribution of Working Group I to the Fifth Assessment Report of the Intergovernmental Panel on Climate Change. Cambridge, United Kingdom and New York, NY, USA: Cambridge University Press, 2013.

[2] IEA, Key World Energy Statistics 2017. 2017.

[3] R. Hull. (2018). Fusioneer list, [Online]. Available: www . fusor . net/board / viewtopic.php?t=13.

[4] High and ultra-high vacuum for science research, Agilent Technologies, 2014. [Online]. Available: https : //www . agilent . com/cs / library/training/ Public/UHV_Seminar_Handbook.pdf.

[5] I. Bello, Vacuum and Ultravacuum. CRC Press, 2017.

[6] C. B. Alcock, V. P. Itkin, and M. K. Horrigan, "Vapour pressure equations for the metallic elements: 298-2500k," Canadian Metallurgical Quarterly, vol. 23, 1984 .

[7] Vapour pressure chart, Luxel Corportation, Washington, U.S.A. [Online]. Available: https : / / luxel . com/wp - content/uploads / 2013/04 / Luxel - Vapor Pressure-Chart.pdf.

[8] W. Umrath, "Fundamentals of vacuum technology," Tech. Rep., 2007. [Online]. Available: https : / / www3 . nd . edu / nssl / Lectures / urls / LEYBOLD_ FUNDAMENTALS . pdf.

[9] S. G. Lias, "Ionization energy evaluation," in NIST Chemistry WebBook, Nist Standard Reference Database, P. J. Linstrom and W. G. Mallard, Eds., 69th ed., 
Gaithersburg, MD, U.S.A: National Institute of Standards and Technology, 2018.

[10] J. A. Geuther, "Radiation generation with pyroelectric crystals," PhD thesis, Rensselaer Polytechnic Institute, 2007.

[11] Y.-K. Kim and K. K. Irikura, "Electron-impact total ionization cross sections of molecular ions," Journal of Research of the National Institute of Standards and Technology, vol. 105, no. 2, 2000.

[12] Y.-K. Kim and M. E. Rudd, "Binary-encounter-dipole model for electron impact ionization," Physical Review A, vol. 50, no. 4, 1994.

[13] K. Wiesemann, "A short introduction to plasma physics," AEPT, Ruhr-Universität Bochum, Germany, Tech. Rep., 2014. [Online]. Available: https://arxiv.org/ ftp/arxiv/papers/1404/1404.0509.pdf.

[14] A. Piel, Plasma Physics, An Introduction to Laboratory, Space, and Fusion Plasmas. Springer, 2010.

[15] T. Kalvas, "Development and use of computational tools for modelling negative hydrogen ion source extraction systems," PhD thesis, University of Jyväskylä, Jyväskylä, 2013.

[16] H. Conrads and M. Schmidt, "Plasma generation and plasma sources," Plasma Sources Science and Technology, vol. 9, no. 4, p. 441, 2000. [Online]. Available: http: //stacks.iop.org/0963-0252/9/i=4/a=301.

[17] J. T. Gudmundsson and A. Hecimovic, "Foundations of de plasma sources," Plasma Sources Science and Technology, vol. 26, no. 12, p. 123001,2017 . [Online]. Available: http://stacks.iop.org/0963-0252/26/i=12/a=123001.

[18] H. W. Loeb, "Plasma-based ion beam sources," Plasma Physics and Controlled Fusion, vol. 47, no. 12B, B565, 2005. [Online]. Available: http://stacks .iop. org $/ 0741-3335 / 47 / i=12 B / a=S 41$. 
[19] R. Scrivens, "Electron and ion sources for particle accelerators," Geneva, Switzerland, Tech. Rep., 2006. [Online]. Available: https : //cds . cern . ch/record/ 941321/files/p495.pdf.

[20] J. R. Pierce, Theory and Design of Electron Beams, second, ser. Bell Telephone Laboratory Series. New York, NY, USA: Van Nostrand, 1954.

[21] R. Becker, "Numerical simulation of ion-beam formation (invited)," Review of Scientific Instruments, vol. 67, no. 3, pp. 1132-1137, 1996. DOI: 10.1063/1. 1146769.

[22] H. Leibl, "Applied charged particle optics," in. Springer, 2008, ch. Lenses: Basic Optics.

[23] T. Kalvas, "Beam extraction and transport," Jyväskylä, Finland, Tech. Rep., 2014. [Online]. Available: https://arxiv.org/pdf/1401 .3951.pdf.

[24] A. Vong Sy, "Advanced penning-type ion source development and passive beam focusing techniques for an associated particle imaging neutron generator with enhanced spatial resolution," PhD thesis, University of California, Berkeley, Berkeley, California, U.S., 2013.

[25] S. S. M. Wong, Introductory Nuclear Physics, 2nd ed. John Wiley and Sons, Inc., 1998.

[26] J. K. Tuli, "Nuclear wallet cards," National Nuclear Data Center, Tech. Rep., 2011. [Online]. Available: http://www.nndc .bnl.gov/wallet/wal135.pdf.

[27] N. Soppera, M. Bossant, and E. Dupont, "Janis 4: An improved version of the nea java-based nuclear data information system," Nuclear Data Sheets, vol. 120, 2014.

[28] P. K. Kaw and I. Bandyopaghyay, "The case for fusion," in Fusion Physics, M. Kikuchi, K. Lackner, and M. Q. Tran, Eds., Vienna, Austria: International Atomic Energy Agency, 2012. 
[29] W. Tornow, S. M. Shafroth, and J. D. Brownridge, "Evidence for neutron production in deuterium gas with a pyroelectric crystal without tip," Journal pf Applied Physics, vol. 104, no. 3, 2008.

[30] B. Naranjo, J. K. Gimzewksi, and S. Putterman, "Observation of nuclear fusion driven by a pyroelectric crystal," Letters to Nature, vol. 434, 2005.

[31] W. Tornow, S. M. Lynam, and S. M. Shafroth, "Substantial increase in acceleartion potential of pyroelectric crystals," Journal of Applied Physics, vol. 107, no. $6,2010$.

[32] J. L. Ellsworth, S. Falabella, S. Sanchez, V. Tang, and H. Wang, "Compact deuterium-tritium neutron generator using a novel field ionization source," Journal of Applied Physics, vol. 116, no. 19, 2014.

[33] J. L. Rovey, "Design parameter investigation of a cold-cathode penning ion source for general laboratory applications," Plasma Source Science and Technology, vol. 17, 2008.

[34] J. L. Rovey, B. P. Ruzic, and T. J. Houlahan, "Simple penning ion source for laboratory research and development applications," Review of Scientific Instruments, vol. 78, 2007.

[35] B. K. Das and A. Shyam, "Development of compact size penning ion source for compact neutron generator," Review of Scientific Instruments, vol. 79, 2008.

[36] J. Long, Z. Yang, P. Dong, X. He, and K. Zhang, "Study on a cold-cathode Hpig-type ion source," Nuclear Science and Techniques, vol. 24, 2013.

[37] Z. Yang, P. Dong, T. Wang, J. Long, C. Lan, X. He, and K. Zhang, "Studies on hydrogen plasma in a penning ion source by optical emission spectroscopy," IEEE Transactions on Plasma Science, vol. 41, no. 10, 2013. 
[38] H. Schulte and B. H. Wolf, "Ion and electron flow in hot-cathode pig sources for multiply charged heavy ions," IEEE Transactions on Nuclear Science, vol. 23, no. $2,1976$.

[39] R. Keller, "Ion extraction systems: Optics and design," Nuclear Instruments and Methods in Physics Research Section A: Accelerators, Spectrometers, Detectors and Associated Equipment, vol. 298, no. 1, pp. 247-254, 1990, ISSN: 0168-9002. DOI: https://doi .org/10.1016/0168-9002(90) 90622-D.

[40] T. Kalvas, O. Tarvainen, T. Ropponen, O. Steczkiewicz, J. Ärje, and H. Clark, "Ibsimu: A three-dimensional simulation software for charged particle optics," Review of Scientific Instruments, vol. 81, no. 2, 2010.

[41] S. B. Bayram and M. V. Freamat, "Vibrational spectra of n2: An advanced undergraduate laboratory in atomic and molecular spectroscopy," American Journal of Physics, vol. 80, no. 8, 2012.

[42] K. Wagatsuma and K. Hirokawa, "Characterization of atomic emission lines from argon, neon and nitrogen glow discharge plasmas," Analytical Chemistry, vol. 57, no. 14,1985 .

[43] J. E. Bringas, Handbook of Comparative World Steel Standards (5th Edition). ASTM Internation, 2016.

[44] J. Donev. (2017). Total primary energy supply, [Online]. Available: www. energyeducation. ca/encyclopedia/Total_primary_energy_supply (visited on 07/02/2018). 


\section{Glossary}

304-SS Type 304 Stainless Steel (304-SS) is the most common SAE International (SAE) designated grade of stainless steel. As with all steel, it primarily contains iron, but also contains $18 \%$ to $20 \%$ chromium, $8 \%$ to $10.5 \%$ nickle, $2 \%$ manganese, and trace amounts of silicon, carbon, phosphorous, sulfur and nitrogen [43]. 8

316-SS Type 316 Stainless Steel (316-SS) is a SAE designated grade of stainless steel. As with all steel, it primarily contains iron, but also contains $16 \%$ to $18 \%$ chromium, $10 \%$ to $14 \%$ nickle, $2 \%$ to $3 \%$ molybdenum, $2 \%$ manganese, and trace amounts of silicon, carbon, phosphorous, sulfur and nitrogen [43]. 8

ANSYS ANSYS Multiphysics is a software suite for multiphysics modelling and simulation. 47

$\mathbf{C}++\mathrm{C}++$ is a programming language that can be used as an object oriented programming language, an imperative programming language, and still provide low-level memory control. Note: All $\mathrm{C}++$ code used in this work is compiled under the $\mathrm{C}++11$ standard. 47, 90, 94, 102

CERN CERN (European Organization for Nuclear Research) is a European research organization that operates many of the world's biggest nuclear and particle physics experiments. 90

CF Conflat Flanges (CF) are flanges suitable for use in constructing both $\mathrm{HV}$ and UHV systems. These flanges are typically made of either 304-SS or 316-SS, and use sex-less mates to create a seal. Both sealing faces have a knife-edge that compresses a gasket made of a softer metal (typically copper) or a vacuum compatible fluorocarbon gasket, thus creating a seal capable of achieving and 
maintaining UHV. The flange's size is designated by a number that follows "CF", which indicates the diameter of the flange in inches. 8

COMSOL COMSOL Multiphysics (COMSOL) is a software package for multiphysics simulation and plotting. 44

Geant4 Geant4 is a toolkit developed by The European Organization for Nuclear Research (CERN) for the simulation of particle-solid interactions. 82

GHG Greenhouse gasses (GHGs) are atmospheric gases which contribute to a global greenhouse effect. These include $\mathrm{CO}_{2}, \mathrm{CH}_{4}, \mathrm{~N}_{2} \mathrm{O}$, and halocarbons such as $\mathrm{CFCl}_{3}, \mathrm{CF}_{2} \mathrm{ClCFCl}_{2}$, and $\mathrm{CHF}_{2} \mathrm{Cl}$ to name primary contributors [1]. 1

HV High Vacuum (HV) is a term which refers to vacuum pressures in the range of $1 \times 10^{-3}$ Torr to $1 \times 10^{-8} \operatorname{Torr}[5] .6$

IBSIMU IBSIMU is a $\mathrm{C}++$ library for ion optics, space-charge calculation, and plasma extraction. It is able to import 2-D and 3-D geometries as exported in .STL or .DXF file formats. It also allows for mathematical definition of geometries. Note: Version 1.0.6 of the IBSIMU library was used for this work [40]. 44, 47-49, 82

ISO The International Organization for Standardization (ISO) is an international body for setting industrial standard. It operates in 162 countries.. 92

JANIS The Java-based Nuclear Data Information System (JANIS) is an NEA maintained data bank containing cross section data for a great number of nuclear interactions, with the impinging particles being neutrons, protons, deuterons, tritons, and major stable helium nuclei. 30

MFP Mean Free Path (MFP) is the average distance travelled by particles between interactions. 7,79 
NEA The Nuclear Energy Agency (NEA) is an international agency under the Organization for Economic Co-operation and Development (OECD) whose stated goal is to promote and assist the peaceful and environmentally friendly use of nuclear energy.. 30

OECD The Organization for Economic Co-operation and Development (OECD) is an international economic organization that promotes democracy and market economy.. 91

PIG A Penning Ion Generator (PIG) is a simple cold cathode plasma ion source. ii, $17,79,81$

PostgreSQL PostgreSQL is an open source relational database that implements SQL. It is known for its close adherence to current SQL standards. Note: Version 10.1 of PostgreSQL was used for this work. 48

Python Python is a flexible scripting language that can be used as an imperative language, an object oriented language. Note: All python code used in this work is written and executed using Python3.6. 46, 47

RGA A Residual Gas Analyzer is a specialized mass spectrometer used in the identification and measurement of constituent gases of the background gas in a vacuum. Most RGAs use quadrupoles as the mass discriminator. ii, 10

SAE SAE International (SAE) is a U.S. based international professional association also responsible for developing many standards used globally. It was started as the Society of Automotive Engineers, hence the initialism that makes up the association's current name. 89

SOLIDWORKS SOLIDWORKS is a CAD tool developed by Dassault Systèmes. Note: Professional Version 2018 was used for this work. 38 
SQL SQL (Structured Query Language) is a language for interaction with relational databases. SQL is defined by an International Organization for Standardization (ISO) standard, but compliance is dependant on the database software provider, and so is not wholly portable between SQL databases. The SQL database used in this work was PostgreSQL version 10.. 48

TFC Total final consumption (TFC) is a measure of consumption of energy in its final state after refinement and conversion [44]. 1

TPES Total primary energy supply is a measure of the total available energy. On a national scale, this would account for imports and exports, but on a global scale represents the amount of energy available before conversion to final use state (refinement for use as fuel, electricity, etc.) [44]. 1

UHV Ultra High Vacuum (UHV) is a term which refers to vacuum pressures less than $1 \times 10^{-8} \operatorname{Torr}[5] .6$ 


\section{Appendix}

\section{A Code}

\section{A.1 Beamline Simulation Suite README}

\section{A.1.1 Introduction}

This is the README for the beamline simulation test suite. The suite was built on CENTOS 7 and so required a number of workarounds in order to make it operational. These may not be necessary on other systems.

\section{A.1.2 Required Packages}

The following are the libraries and packages used to create the test suite.

- IBSIMU version 1.0.6-dev for the beamline simulation. It was manually installed from the sourceforge git repository located at https://sourceforge.net/pr ojects/ibsimu/files/ibsimu/ Additional information on dependencies and installation guide can be found at http://ibsimu. sourceforge.net/install ation.html. All dependencies were installed via the package manager yum and not manually.

- Python 3.6 installed via yum by manually adding a repo. The guide for doing this is from https://janikarhunen.fi/how-to-install-python-3-6-1-on - centos-7.html and the required python modules (installed via pip) are as follows

- psycopg2

- datetime (should be pre-installed with python3.x)

- yaml (should be pre-installed with python3.x) 
- multiprocessing (should be pre-installed with python3.x)

- BOOST 1.66 $\mathrm{C}++$ Libraries installed manually from source and configured to run with python 3.6

- Postgresql10 installed via yum repository and setup using the guide found here: https://linode.com/docs/databases/postgresql/how-to-installpostgresql-relational-databases-on-centos-7/

- Additional information on enabling connections through python available here https://www.depesz.com/2007/10/04/ident/

\section{A.1.3 Installation and Setup Process}

- IBSIMU 1.0.6 dev

- The installation process for IBSIMU 1.0.6dev is started by downloading the .tar.gz files from https://sourceforge.net/projects/ibsimu/file s/ibsimu/ and extracting them. This was done in the following location $/ \mathrm{src} /$

- From within the extracted folder, the process was started by running the following commands

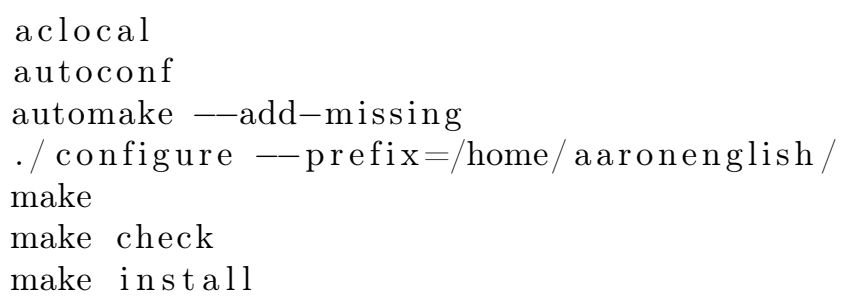

- The first few commands aren't necessarily required, but may be useful in resolving some issues. The dev branch was chosen for the installation to be able to take advantage of the modern incarnation of suitesparse (or specifically umfpack from within suitesparse). 
- Once the installation was completed, the following lines were added to /.bashrc so that the IBSIMU libraries could be found on compilation. export PKG_CONFIG_PATH="PKG_CONFIG_PATH: / lib / pkgconfig " export LD_L̈BRARY_PATH="\$LD_L̈BRARY_PATH: / $1 \mathrm{ib} "$

- Ensure that the makefile points to the right name of IBSIMU

- Python3.6

- First, installation of some base packages must be verified by running the following commands

sudo yum update

sudo yum install

sudo yum groupinstall development

- Then an additional repository must be added

sudo yum install https:// centos 7 .iuscommunity.org/ius-release . $\mathrm{rpm}$

- Now python 3.6 can be installed

sudo yum install python $36 u$

- Now development headers and pip must be installed

sudo yum install python $36 u-p i p$

sudo yum install python $36 u-$ devel

- For good measure a virtual environment will be created within which all additional pip packages will be installed

python $3.6-\mathrm{m}$ venv venv

- This can then be activated or deactivated at any time by the following commands

. /venv/bin/activate

deactivate

- IMPORTANT NOTE: For the purposes of boost, it is important to make note of the location of the Python.h header file as well as the location 
of the lib. Following these instructions the python root was found to be in /usr/lib64/python3.6 and NOT in lib/. Additionally, the python executable was in /usr/python3.6m. These were all found to cause no issues so long as the makefile and the boost setup were done using the correct and matching paths.

\section{- BOOST 1.66}

- First the .tar.gz must be downloaded and extracted. In this case, it was downloaded and extracted to $\sim /$ src/

- From within the uncompressed folder /src/boost1.66, the following commands must be run:

\footnotetext{
./ bootstrap.sh - prefix $=/$ home/aaronenglish - - with - python $=/$ usr / python $3.6 \mathrm{~m}-$-with - python - version $=3.6-$ - with - python - root $=$ / usr/lib64/python3.6
}

- This creates a project-config.jam file which contains the paths to the python installation, as well as the path that the libraries will be copied to .$/$ b2 install

- This will execute the moving of the libraries to the appropriate locations. Once these have been completed, the libraries are ready to be used. See the makefile for the required compilation structure

- If issues are being encountered, try adding the following to the /.bashrc export CPLUS_INCLUDE_PATH=" $\$$ SCPLUS_INCLUDE_PATH: / us r / include / python $3.6 \mathrm{~m} /:^{\sim} / \operatorname{src} /$ boost_1_66_0"

- Postgresql10

- Install all the key postgresql files as well as dependencies sudo yum install postgresql10-server
sudo yum install postgresql10-contrib

- Initialize the database, start postgresql and allow it to start on startup. 
sudo postgresql-setup initdb

sudo systemctl start postgresql

sudo systemctl enable postgresql

- Set the password for the special postgres user that was created by the installation and change to that user.

sudo passwd postgres

su $*$ postgres

- Change the postgresql internal user password and start the postgresql Command Line Interface (CLI).

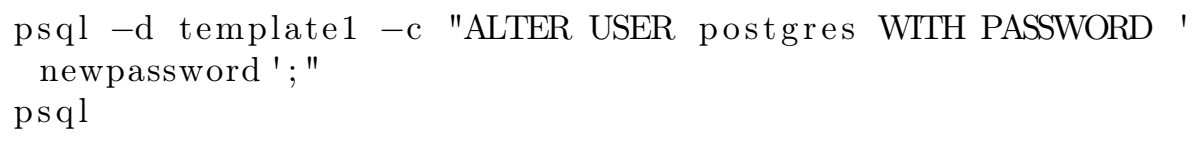

- From within the postgresql CLI identify the location of the hba config file in order to modify the connection permissions.

show hba_file;

$\backslash \mathrm{q}$

- Edit the config file at the following lines with the listed info in order to allow connection to the database via python. Note: this must be done as the postgres user and the objective here is to edit the hba config file to enable connection to the db through python

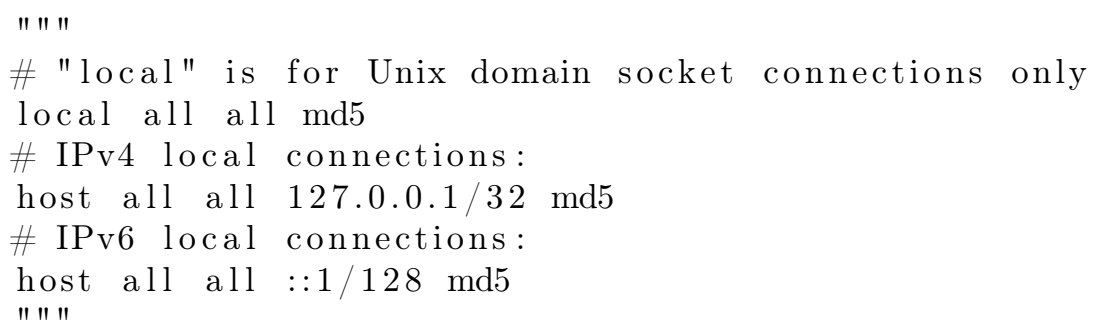

- The database must now be restarted for these changes to take effect but the location of pg_ctl must be specified as it is not in the path. In this install it was located and thus could be run by first changing directory and then executing the pg_ctl command as shown below.

cd / usr/pgsql - 10/bin

. / pg_ctl restart 
- From here postgresql is good to go. The following commands were used to initialize the database used for the experiments.

createdb beamSims

- All subsequent commands were executed from within the postgresql CLI.

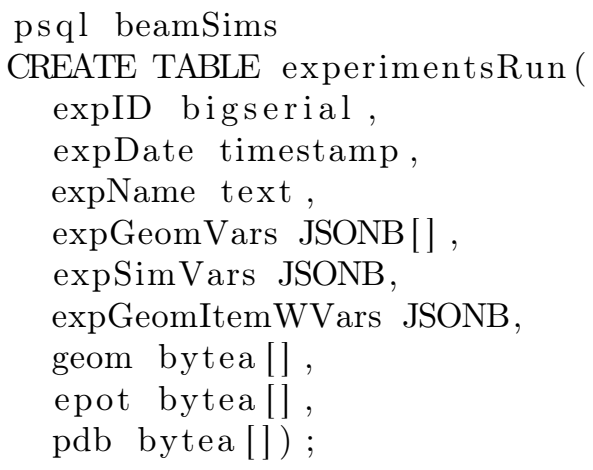

\section{A.1.4 Code Hierarchy}




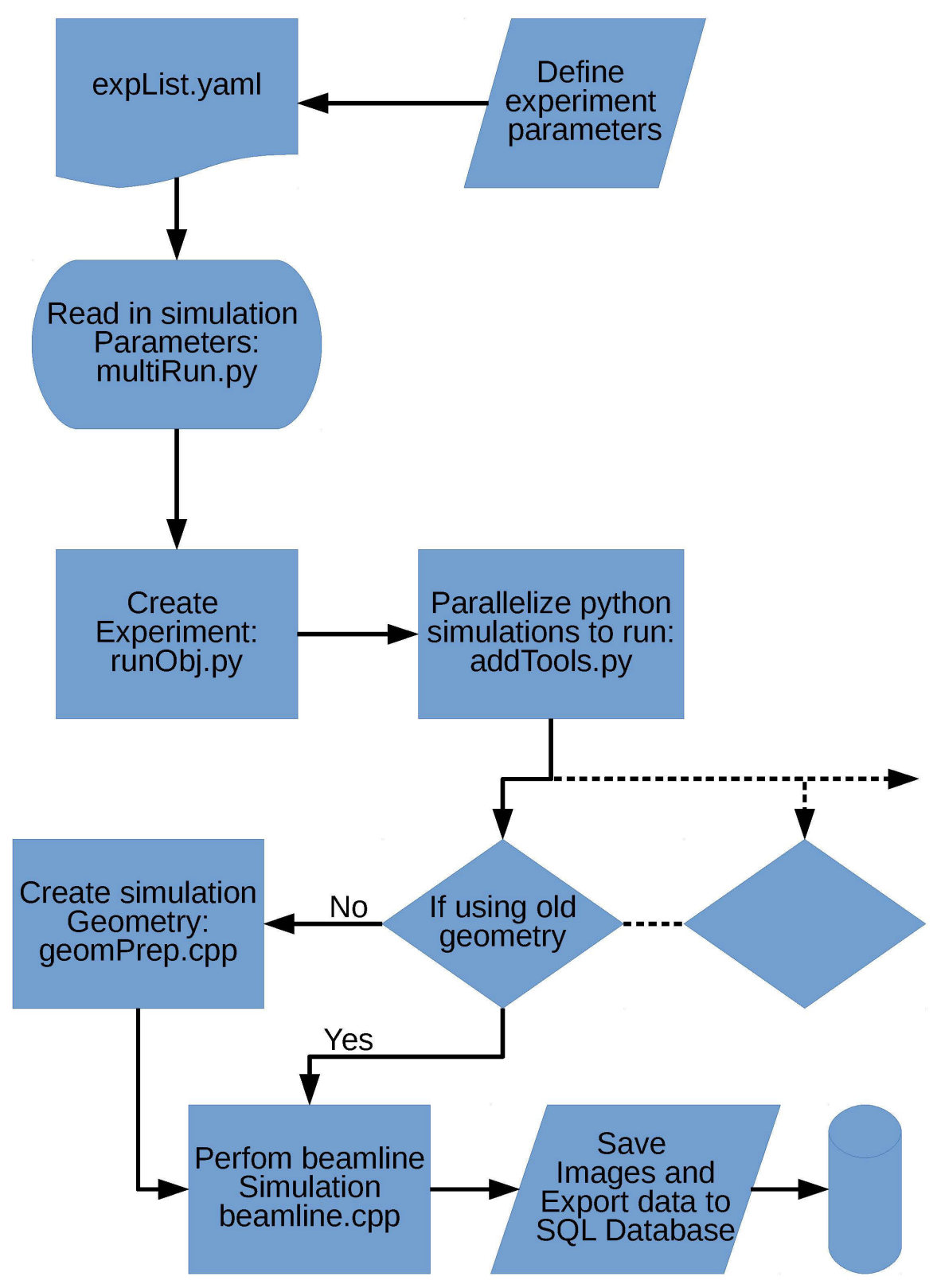

Figure 50: Flow chart for beamline simulation code 


\section{A.1.5 Usage}

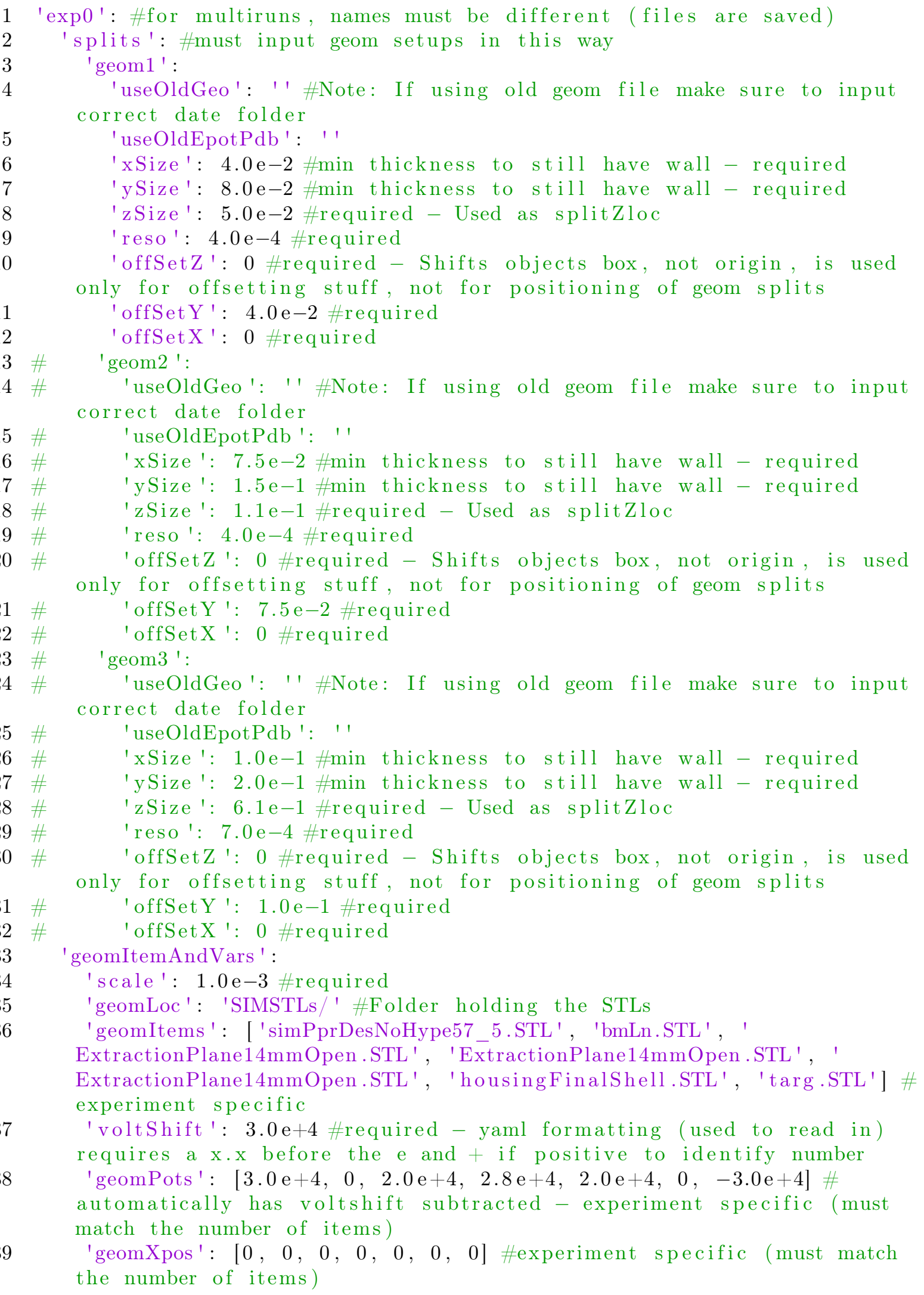




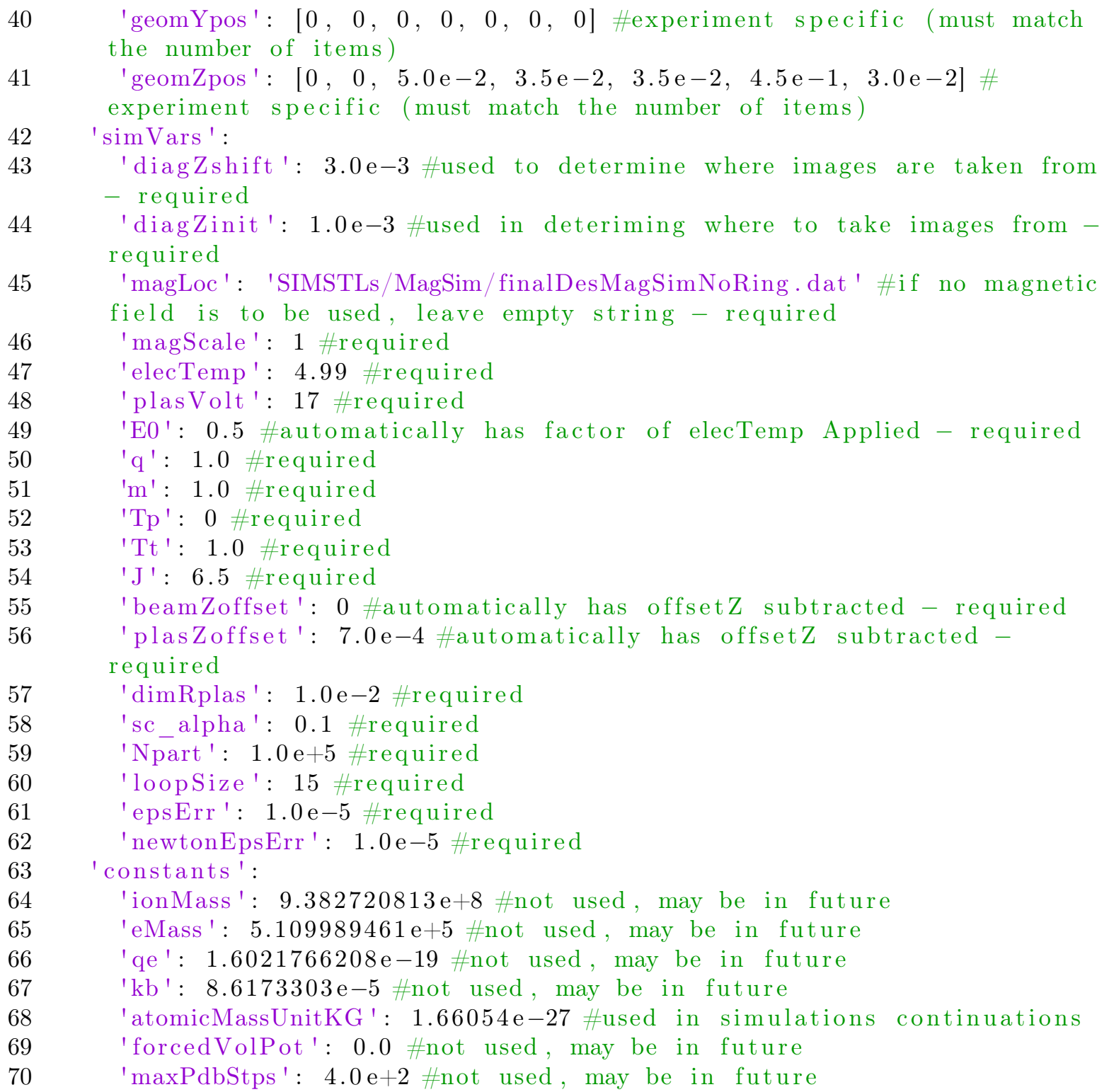

Listing 1: expList.yaml

- All experiments to be run are defined from the yaml file titled expList

- When initiated, the yaml file is read in by the python code multiRunPy and is parsed into a python dict

- This dict contain each run to be done, within which are nested dicts which are the simulation variables, geometry variables, and the geometry item variables

- These dicts are then fed into python objects which hold all the data and results 
- The objects are then passed to the $\mathrm{C}++$ code geomPrep to create the geometry files

- Once completed the geometry variables of that successful run (and the resultant geom file) are fed into a new row of the database and stored in a local folder

- The objects are then passed to the beamline $\mathrm{C}++$ code to have the EM simulation performed

- These beamline simulations run in parallel thanks to python's multiprocessing module

- The beamline simulations once completed have the simulation variables as well as the resultant epot, pdb, and key image files fed into the sql database and saved in the appropriate local folder

- These simulations can then be explored in depth using the analysis $\mathrm{C}++$ code

\section{A.2 Beamline Simulation Code}

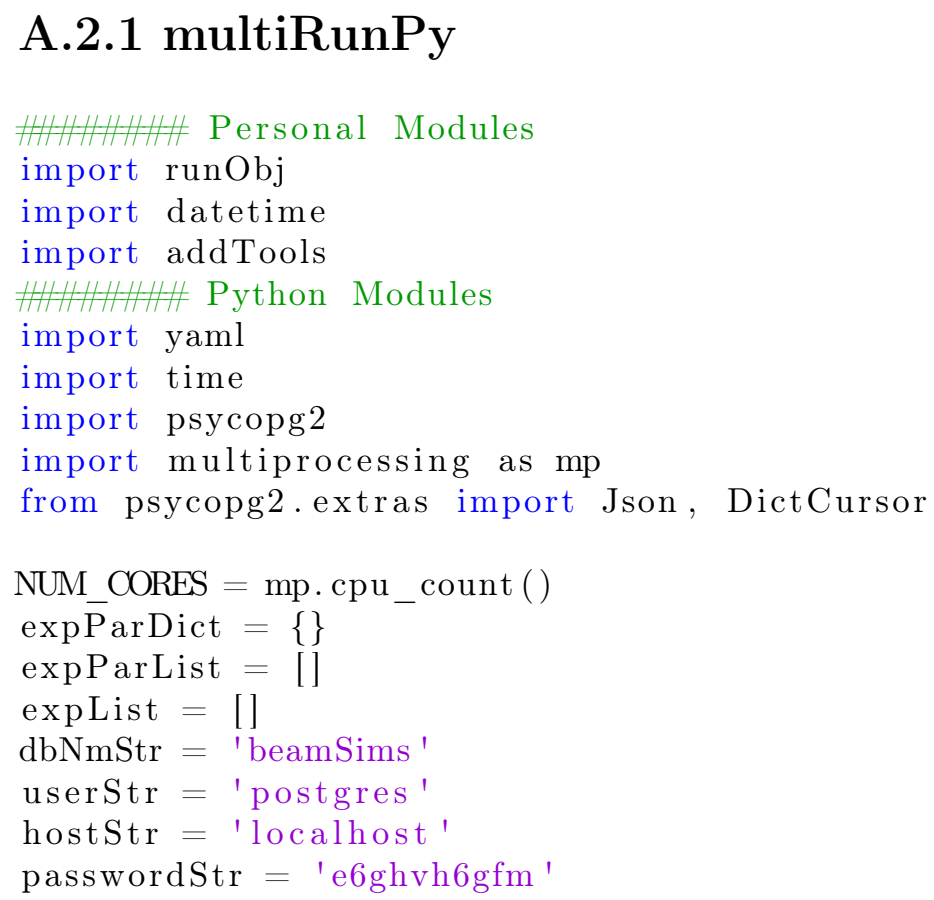




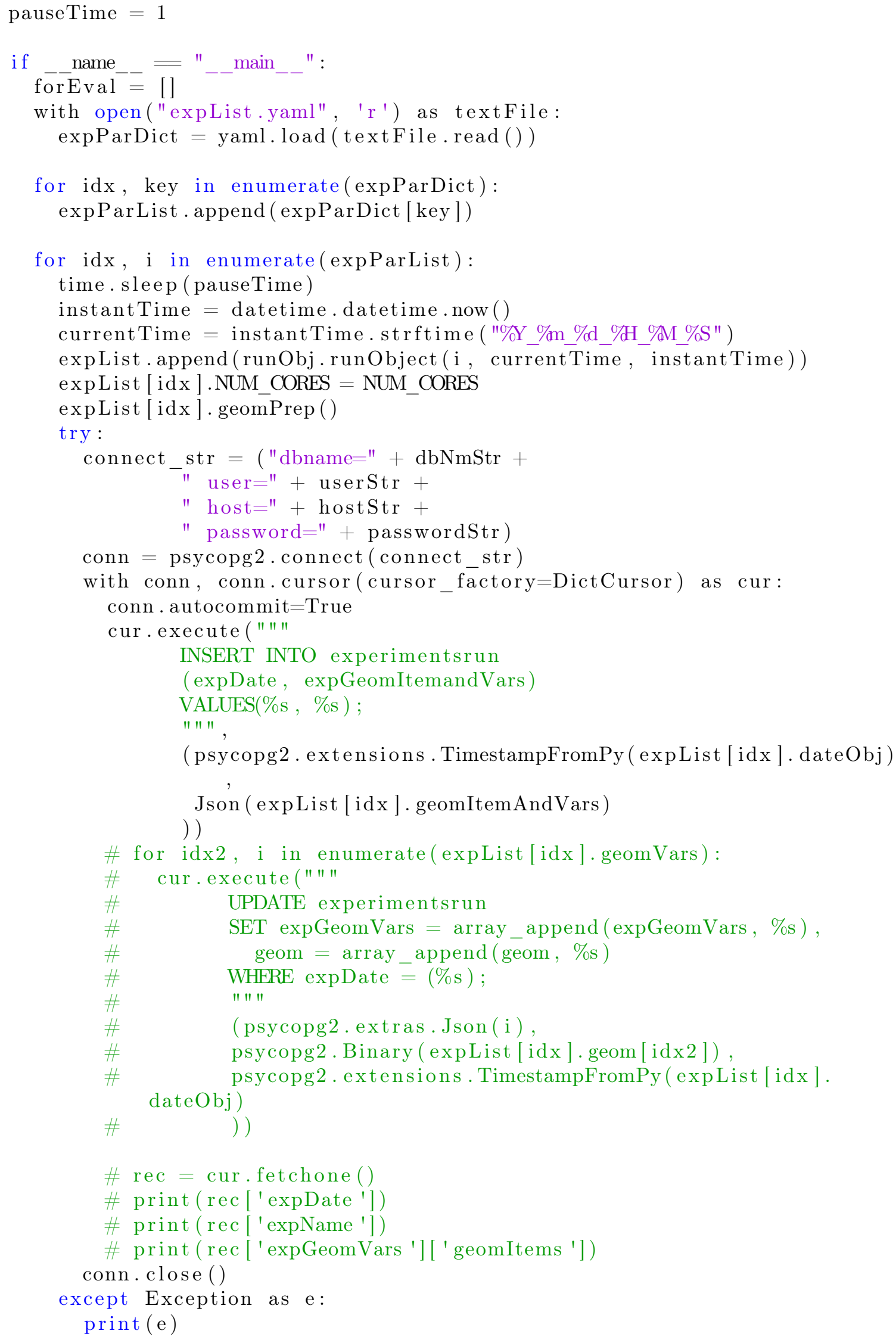


72

73

74

75

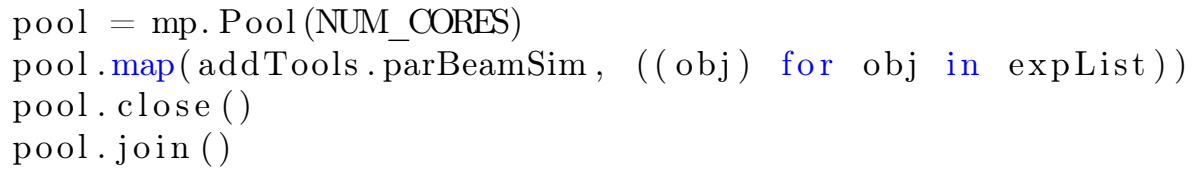

Listing 2: multiRunPy.py

\section{A.2.2 runObj}

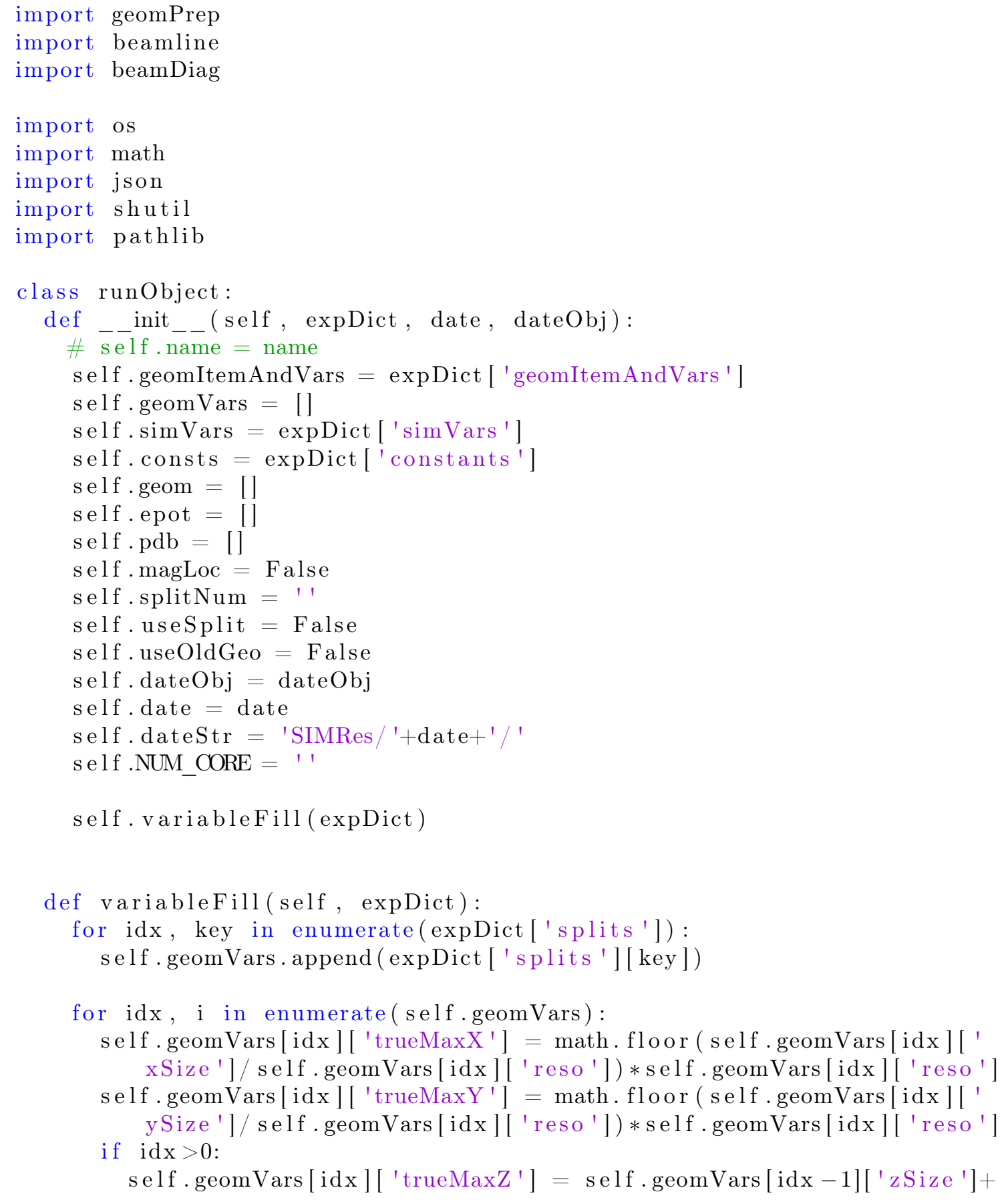


math.floor (( self.geomVars [idx]['zSize ']-self.geomVars [idx $-1][$ 'zSize'])/

else : self geomVars [idx ] ['reso']) *self geomVars [idx ] ['reso']

self.geomVars[idx]['trueMaxZ'] = math.floor(self.geomVars[idx][' zSize']/self.geomVars[idx]['reso'])*self geomVars[idx]['reso ']

print ( self .geomVars [idx ] [ 'trueMaxX'], self .geomVars [idx ]['trueMaxY' ], self .geomVars [idx ] ['trueMaxZ'])

def geomPrep(self):

for idx, i in enumerate(self.geomItemAndVars['geomItems ']): self.geomItemAndVars ['geomItems' $][\mathrm{idx}]=$ self.geomItemAndVars [' geomLoc']+i \#modifying the file names to point to the folder STLs/

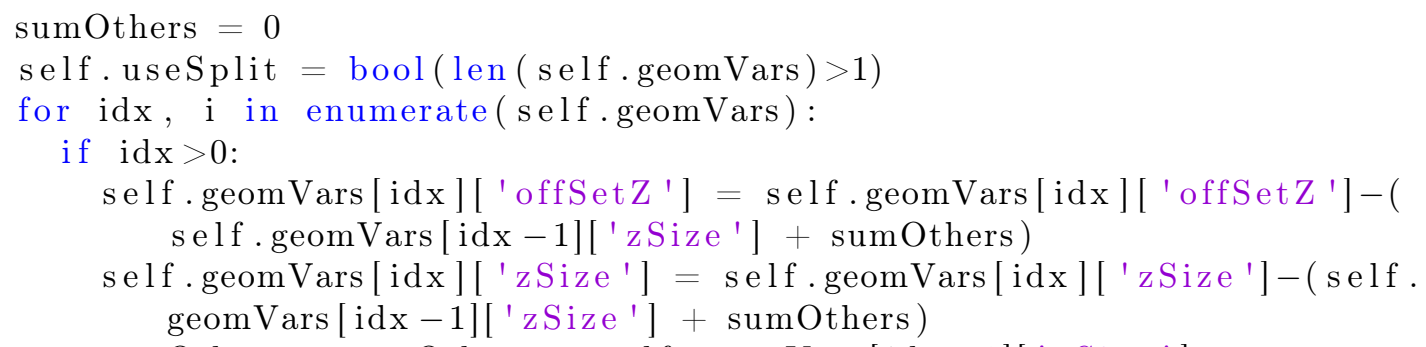




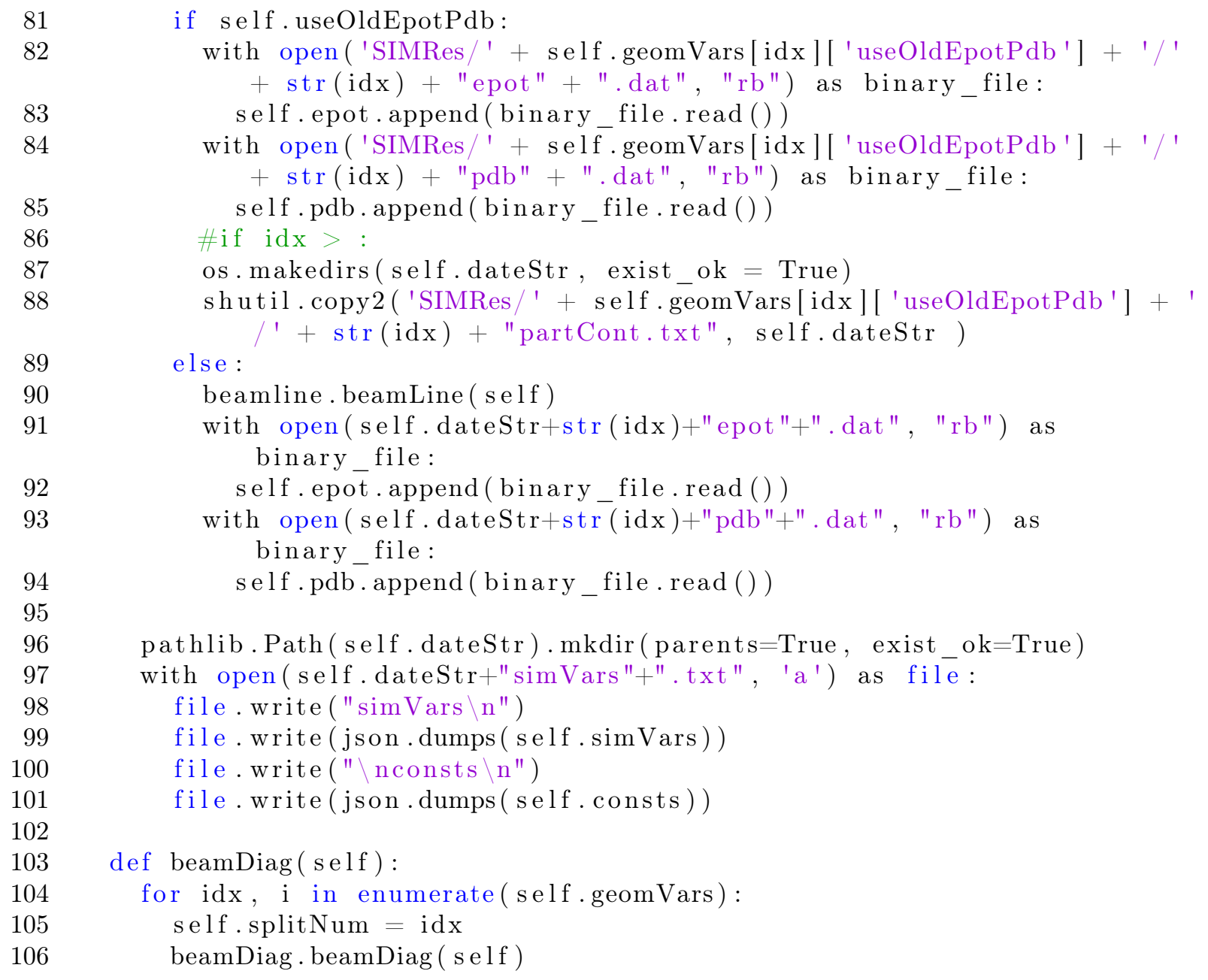

Listing 3: runObj.py

\section{A.2.3 addTools}

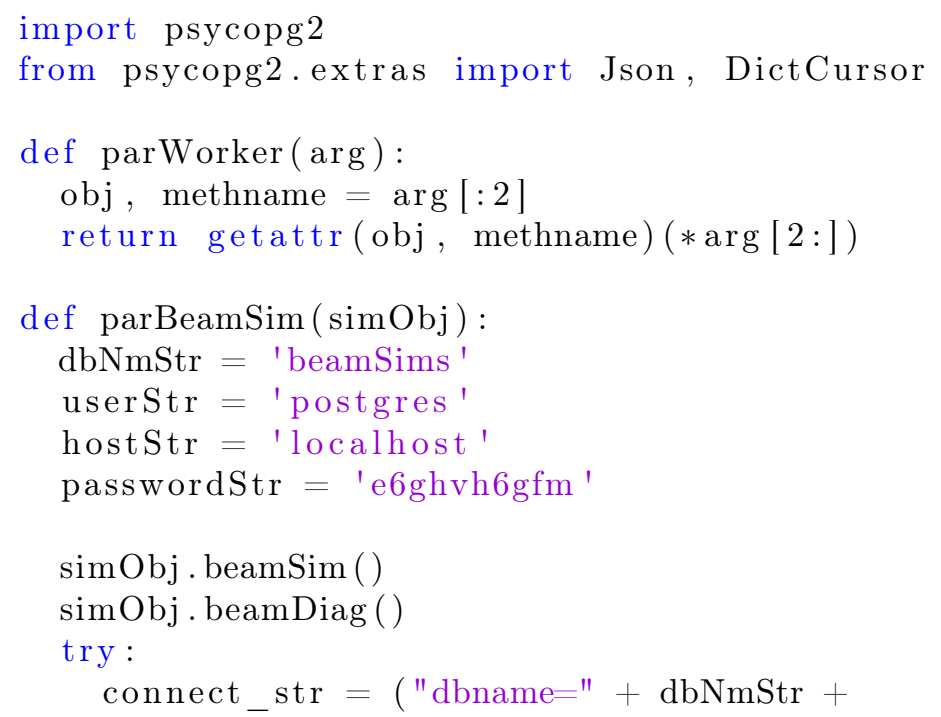




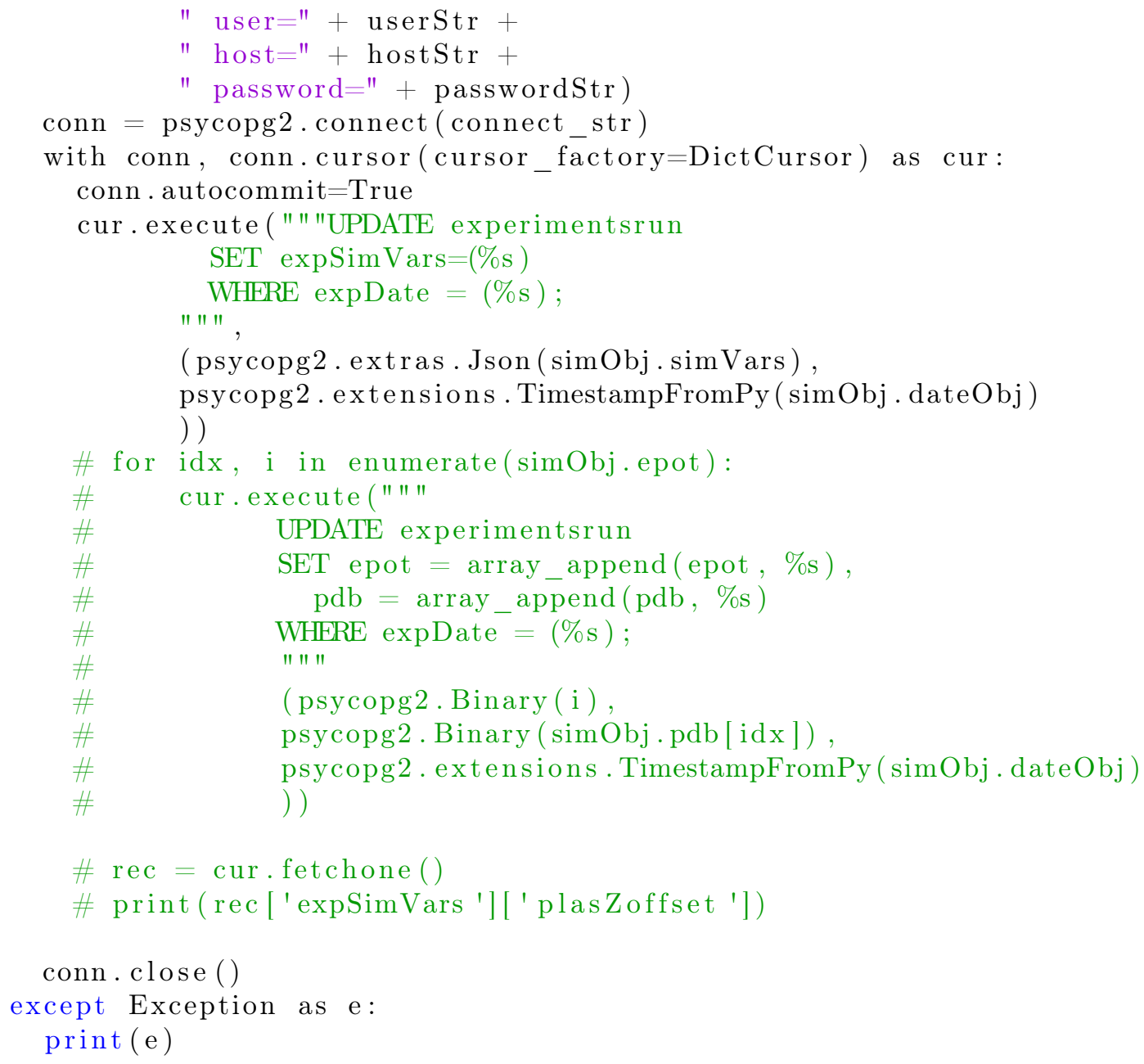

Listing 4: addTools.py

\section{A.2.4 geomPrep}

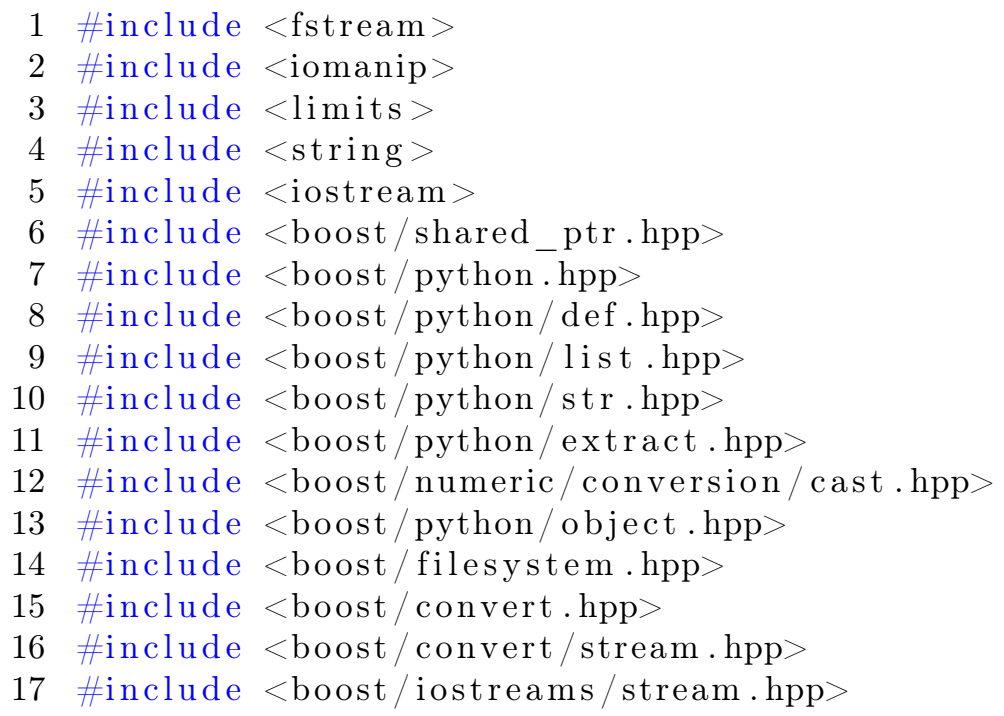




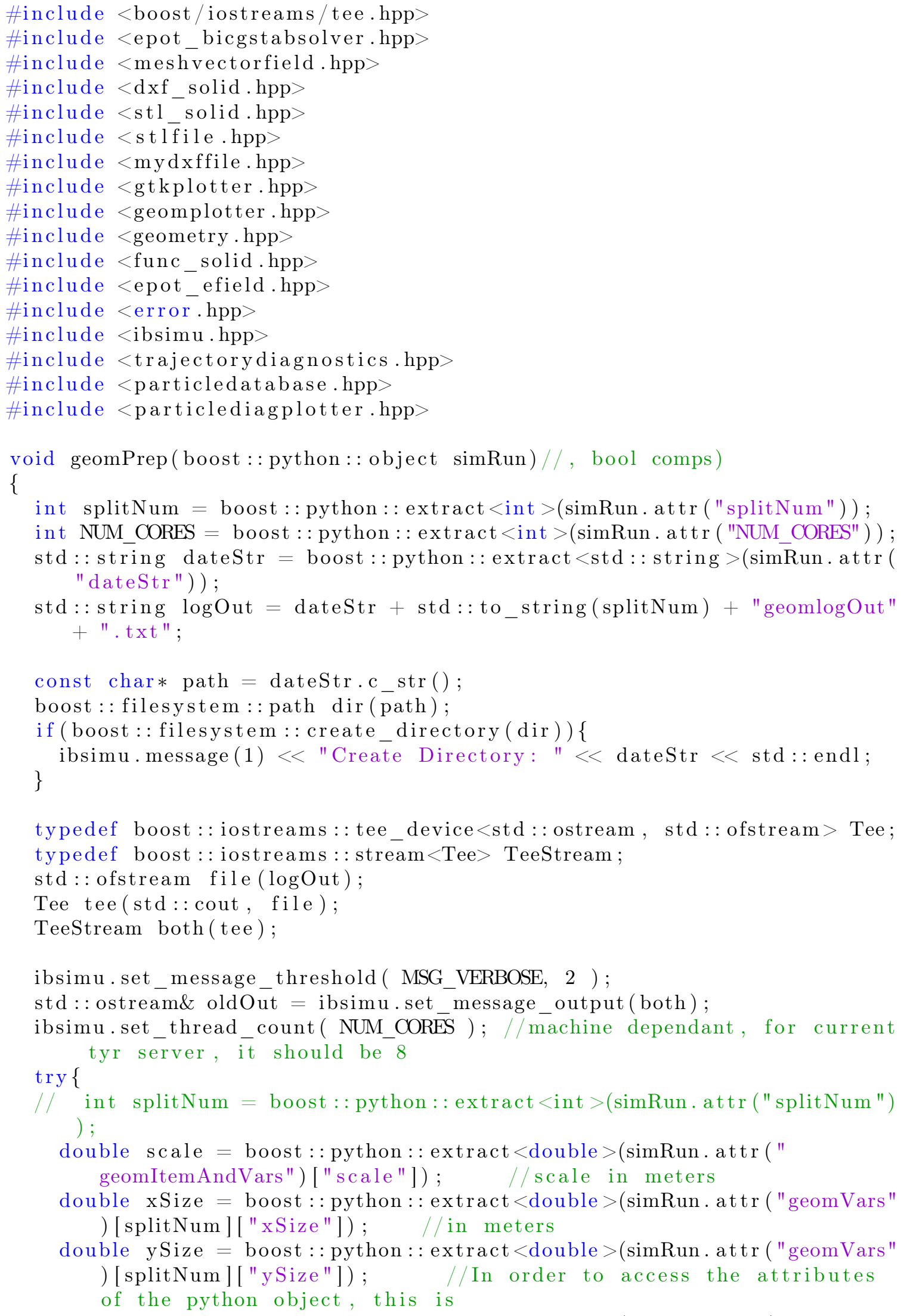


) [splitNum]["zSize"]); //the code thats required. It must further be extracted into a $c++$ typ

double offSetX = boost : : python : : extract $<$ double $>$ (simRun. attr $("$ geomVars") [splitNum ] ["offSetX"]); // any offset resulting from the model itself

double offSetY $=$ boost : : python : : extract $<$ double $>$ (simRun attr $("$ geomVars") [splitNum ] ["offSetY"]); //These values were found using the wqindows

double offSetZ = boost : : python : : extract $<$ double $>$ (simRun . attr $("$ geomVars") [splitNum ] ["offSetZ"]); // built in stl viewer which would display all double voltShift $=$ boost : : python : : extract $<$ double $>$ (simRun.attr $("$ geomItemAndVars") [ "voltShift"]);

double reso $=$ boost : : python : : extract $<$ double $>$ (simRun attr $(" \operatorname{geomVars"})$ [splitNum]["reso"]); //size of nodes in meters

int itemCount $=7$;

$/ /$ std : : string expName = boost : : python : : extract $<$ std : : string $>(\operatorname{simRun}$. $\operatorname{attr}($ "name"));

std:: string dateStr = boost: : python : : extract $<$ std : : string $>$ (simRun. $\operatorname{attr}(" d \operatorname{ateStr")})$;

$/ / \operatorname{const} \operatorname{char} * \operatorname{path}=$ dateStr.c $\_$str ()

boost:: filesystem : : path dir (

if (boost : : filesystem : : create_directory (dir)) \{

ibsimu.message $(1)<<$ "Create Directory: " $<<$ dateStr $<$ std: : endl;

std:: string geoNm $=$ dateStr + std : : to_string $(\operatorname{splitNum})+$ "geom" + ". dat" ;

Int3D meshSize( ( int ) floor $(x \mathrm{Size} / \mathrm{reso})+1$, (int) floor (ySize/reso $)+1$, (int) floor (zSize/reso $)+1$ ); // calculation gives number of nodes in each dimension

Vec3D origin (- offSetX, - offSetY, - offSetZ );

Transformation $\mathrm{t}$;

t.scale( Vec3D( scale, scale, scale) ); //Scale is defined by user, not stl file

Geometry geom( MODE_3D, meshSize, origin, reso );

geom.set_boundary ( 1 , Bound(BOUND_NEUMANN, 0.0));

geom.set_boundary ( 2 , Bound(BOUND_NEUMANN, 0.0));

geom.set_boundary ( 3 , Bound(BOUND_NEUMANN, 0.0));

geom.set_boundary ( 4 , Bound(BOUND_NEUMANN, 0.0));

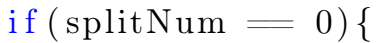

geom.set_boundary ( 5, Bound(BOUND_DIRICHLET, 0.0));

\} else \{

\}

geom.set_boundary ( 5, Bound(BOUND_NEUMANN, 0.0));

geom.set_boundary ( 6, Bound(BOUND_NEUMANN, 0.0) ); 


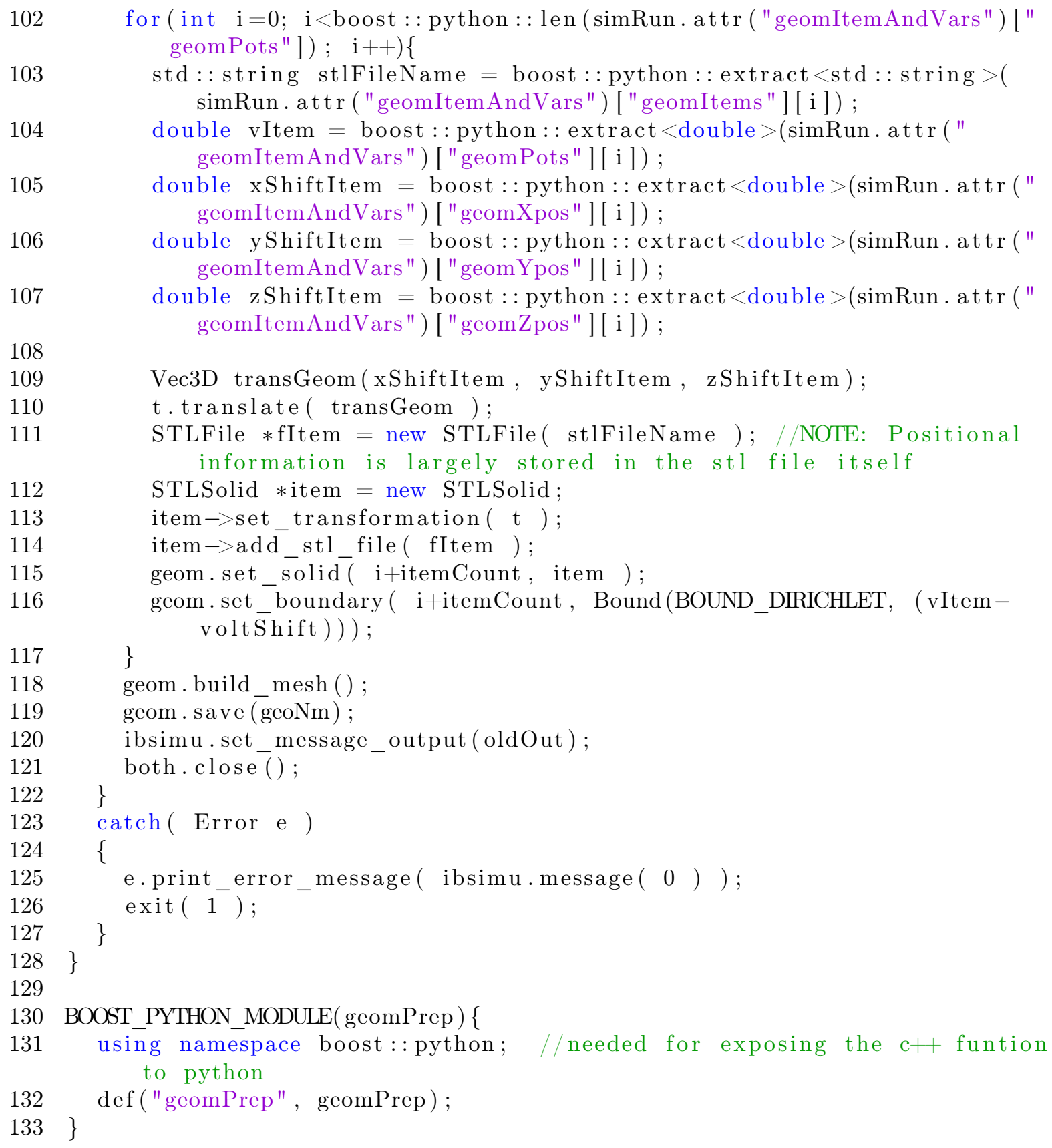

Listing 5: geomPrep.cpp

\section{A.2.5 beamline}

1 \#include <fstream $>$

2 \#include <iomanip $>$

3 \#include $<$ limits $>$

4 \#include $<$ cmath $>$

5 \#include $<$ string $>$

6 \#include <iostream >

7 \#include <boost/python.hpp $>$ 


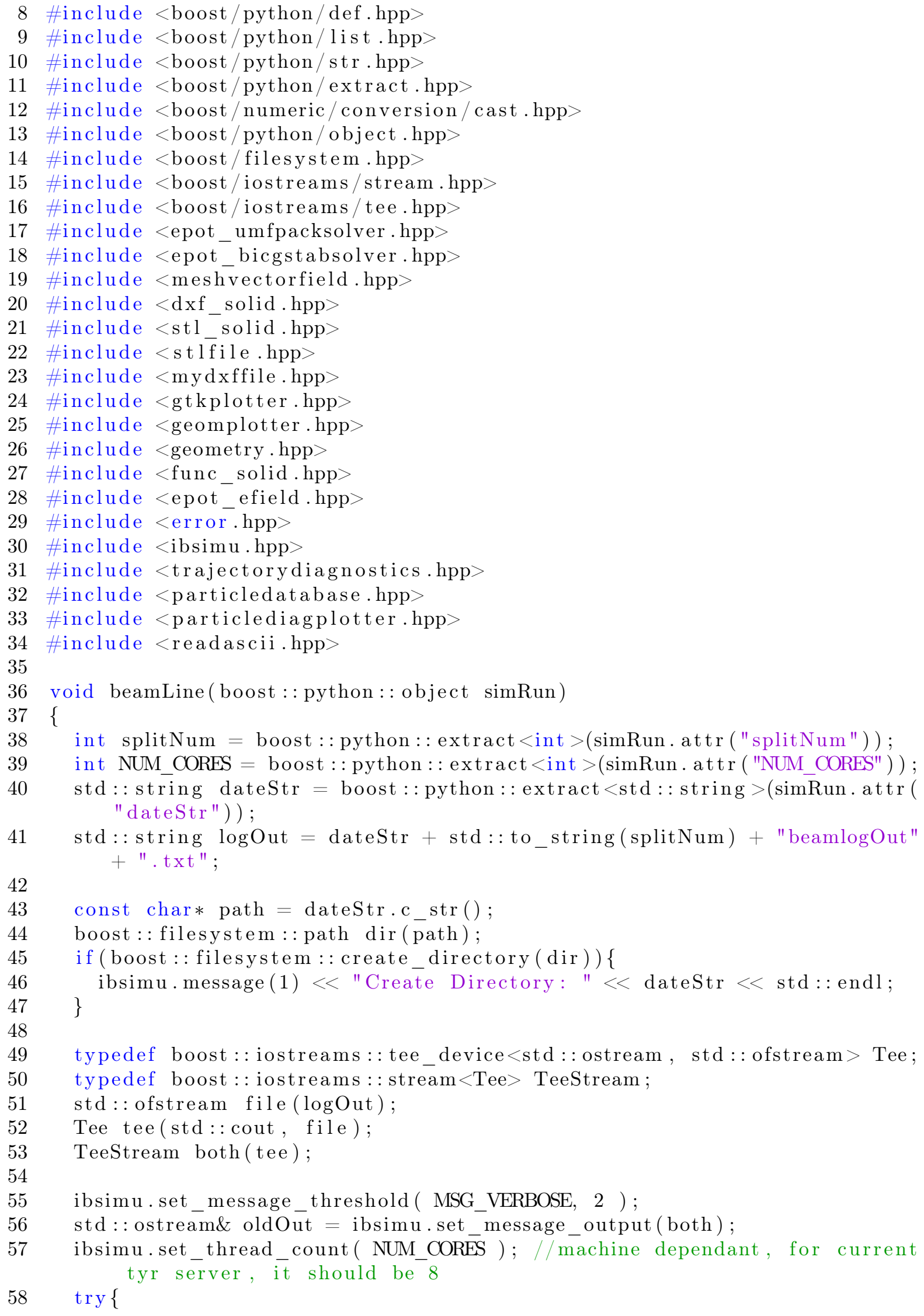


double offSetX $=$ boost:: python: : extract $<$ double $>$ (simRun. attr $("$ geomVars") [splitNum][" offSetX "]); // any offset resulting from the model itself $/ /$ double offSetY $=$ boost $:$ : python : : extract $<$ double $>$ (simRun. attr $("$ geomVars") [splitNum][" offSetY"]); // These values were found using the wqindows

double offSetZ = boost:: python: : extract $<$ double $>$ (simRun.attr $("$ geomVars") [ splitNum ] [ offSetZ"]); double $z$ Size $=$ boost:: python : : extract $<$ double $>$ (simRun.attr $($ "geomVars" ) [splitNum]["zSize"]); //the code thats required. It must further be extracted into a $\mathrm{c}++$ typ double zSizePrev $=0$;

double plasVolt = boost:: python: : extract $<$ double $>$ (simRun.attr $("$ simVars") ["plasVolt"]); // the plasma voltage simVars")["elecTemp"]); // electron temperature in the parrallel direction ) ["E0"]); // beam energy double $\mathrm{E} 0=$ E0Temp*elecTemp;

double $q=$ boost : : python : : extract $<$ double $>$ (simRun. attr $(" \operatorname{sim} V \operatorname{ars} ")[$ "q" ]); //charge in units of $q$

double $\mathrm{m}=$ boost : : python : : extract $<$ double $>$ (simRun. attr ( "simVars") [ "m" ]); //mass of particles in beam (in atomic units) double $\mathrm{Tp}=$ boost : : python : : extract $<$ double $>$ (simRun.attr ("simVars") [" $\mathrm{Tp} "])$;

double $\mathrm{Tt}=$ boost $:$ : python $:$ : extract $<$ double $>$ (simRun. attr $(" \operatorname{sim}$ Vars" $)$ [" Tt"]); //transverse plasma electron temperature

double $\mathrm{J}=$ boost : : python : : extract $<$ double $>$ (simRun. attr $(" \operatorname{sim} V \operatorname{ars}$ ") [ "J" ]) ; //Current density of beam in $\mathrm{A} / \mathrm{m}^{\wedge} 2$

double beamZoffsetTemp = boost: : python : : extract $<$ double $>$ (simRun $\cdot$ attr ( "simVars") [ "beamZoffset"]);

double beamZoffset $=$ beamZoffsetTemp - offSetZ;

double dimRplas $=$ boost : : python : : extract $<$ double $>$ (simRun. attr $("$ simVars") [ "dimRplas"]);

double NpartTemp = boost:: python: : extract $<$ double $>$ (simRun.attr $("$ simVars") [ "Npart"]);

int Npart $=$ boost : : numeric_cast $<$ int $>($ NpartTemp $)$;

double loopSizeTemp = boost : : python: : extract $<$ double $>$ (simRun. attr (" simVars") [ "loopSize"]);

double epsErr $=$ boost : : python : : extract $<$ double $>$ (simRun.attr $(" \operatorname{simVars"}$ ) ["epsErr"]);

double newtonEpsErr $=$ boost : : python : : extract $<$ double $>$ (simRun. attr $("$ simVars") [ "newtonEpsErr"]);

uint loopSize = boost: : numeric_cast $<$ uint $>($ loopSizeTemp $)$;

double scale $=$ boost: : python: : extract $<$ double $>$ (simRun.attr $("$ geomItemAndVars") ["scale"]);

double magScale $=$ boost : : python : : extract $<$ double $>$ (simRun. attr $("$ simVars") ["magScale"]); 
92

93

94

95

96

97

98

99

100

101

102

103

104

105

106

107

108

109

110

111

112

113

114

115

116

117

118

119

120

121

122

123

124

125

126

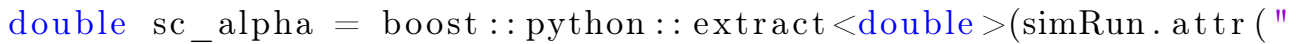
simVars") ["sc_alpha"]);

double plasZoffsetTemp = boost: : python: : extract $<$ double $>$ (simRun attr ( "simVars") ["plasZoffset"]);

double plasZoffset $=$ plasZoffsetTemp - offSetZ;

bool useMag = boost: : python : : extract $<$ bool $>(\operatorname{simRun}$. attr $($ "useMag" $))$;

bool useOldGeo = boost: : python : : extract $<$ bool $>$ (simRun.attr ("useOldGeo ")) ;

double atomicMassUnitKG $=$ boost : : python : : extract $<$ double $>$ (simRun attr ("consts") ["atomicMassUnitKG"]);

double trueMaxZ = boost:: python : : extract $<$ double $>$ (simRun.attr $("$ geomVars") [ splitNum ] [ "trueMaxZ"]);

$/ /$ std : : string expName $=$ boost $:$ : python $:$ : extract $<$ std $:$ : string $>($ simRun . $\operatorname{attr}($ "name"));

if $($ splitNum $>0)\{$

zSizePrev = boost : : python $:$ : extract $<$ double $>$ (simRun. attr $($ "geomVars" $)$ [splitNum -1]["zSize"]);

\}

Vec3D dir $1(1,0,0)$;

Vec3D dir $2(0,1,0)$;

Vec3D blOrigin $(0,0$, beamZoffset $)$;

ibsimu.message $(1)<<$ splitNum $<<$ " Z offset $="<<$ beamZoffset $<<" \backslash$ $\mathrm{n} "$

$/ /$ const $\operatorname{char} *$ path $=$ dateStr.c_str ()$;$

boost:: filesystem : : path dir ( path);

if (boost: : filesystem : : create_directory (dir)) \{

ibsimu.message $(1)<<$ "Create Directory: " $<<$ dateStr $<<$ std:: endl ;

/ $\}$

std:: string epotNm $=$ dateStr + std::to_string (splitNum) + "epot" + ". dat" ;

std:: string pdbNm = dateStr + std::to_string( dat" ;

std:: string geomNm $=$ dateStr + std::to_string( splitNum) + "geom" + ". dat";

std: : string emitNm $=$ dateStr + std:: to_string $($ splitNum $)+$ "emit" + ". txt" ;

std::string partContNm $=$ dateStr + std::to_string $($ splitNum $)+$ " partCont"+ ".txt";

std : : istringstream datStream (boost : : python : : extract $<$ std : : string $>($ simRun.attr ("geom") [ splitNum]));

std:: string partContPrevNm = dateStr + std : : to_string $($ splitNum -1$)+$

"partCont" + ".txt";

Geometry geom( datStream );

geom.build_surface (); 


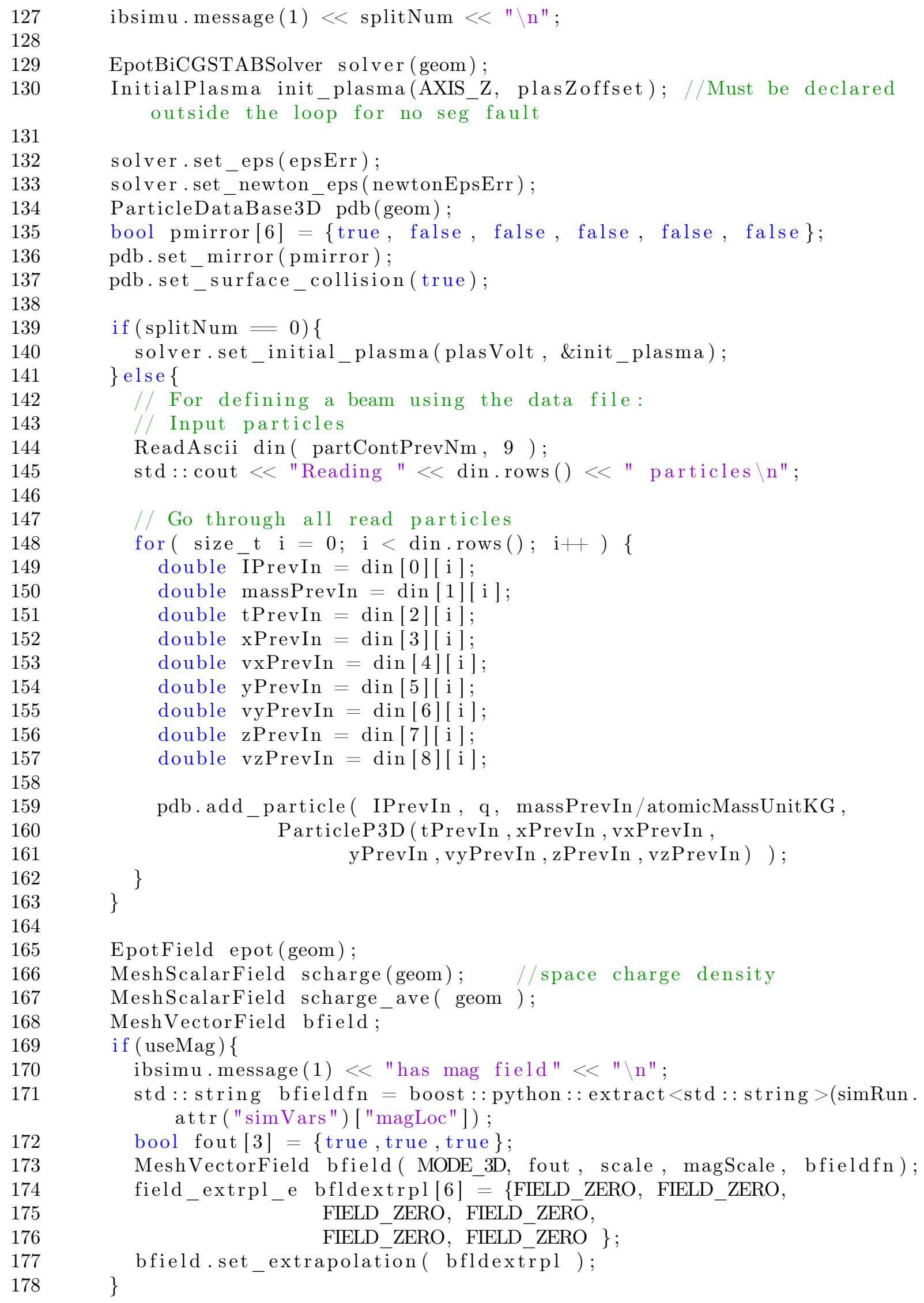

ibsimu. message $(1)<<$ splitNum $<<" \backslash \mathrm{n} "$

EpotBiCGSTABSolver solver (geom);

InitialPlasma init_plasma(AXIS_Z, plasZoffset); //Must be declared outside the loop for no seg fault

solver.set_eps (epsErr);

solver.set_newton_eps (newtonEpsErr);

ParticleDataBase3 $\overline{\mathrm{D}}$ pdb(geom);

bool pmirror $[6]=\{$ true, false, false, false, false, false $\}$;

pdb.set_mirror (pmirror);

pdb.set_surface_collision (true);

if ( splitNum $=0)\{$

solver.set_initial_plasma(plasVolt, \&init_plasma);

\} else \{

$/ /$ For defining a beam using the data file:

$/ /$ Input particles

ReadAscii din( partContPrevNm, 9 );

std: : cout $<$ "Reading " $<$ din.rows ()$<<$ " particles $\backslash$ n";

$/ /$ Go through all read particles

for $\left(\operatorname{size} \mathrm{t}_{\mathrm{i}}=0 ; \mathrm{i}<\right.$ din.rows ()$\left.; \mathrm{i}++\right)\{$

double IPrevIn $=\operatorname{din}[0][\mathrm{i}]$;

double massPrevIn $=\operatorname{din}[1][\mathrm{i}]$;

double tPrevIn $=\operatorname{din}[2][\mathrm{i}]$;

double $x$ PrevIn $=\operatorname{din}[3][\mathrm{i}]$;

double vxPrevIn $=\operatorname{din}[4][\mathrm{i}]$;

double yPrevIn $=\operatorname{din}[5][\mathrm{i}]$;

double vyPrevIn $=\operatorname{din}[6][\mathrm{i}]$;

double zPrevIn $=\operatorname{din}[7][\mathrm{i}]$;

double vzPrevIn $=\operatorname{din}[8][\mathrm{i}]$;

pdb.add_particle(IPrevIn, q, massPrevIn/atomicMassUnitKG, ParticleP3D (tPrevIn, xPrevIn, vxPrevIn, yPrevIn, vyPrevIn, zPrevIn , vzPrevIn ) ) ;

\}

\}

EpotField epot(geom);

MeshScalarField scharge(geom); //space charge density

MeshScalarField scharge_ave( geom );

MeshVectorField bfield;

if ( useMag) \{

ibsimu. message $(1)<<$ "has mag field" $<<" \backslash \mathrm{n} "$;

std: : string bfieldfn = boost : : python : : extract $<$ std : : string $>(\operatorname{simRun}$. attr ("simVars") ["magLoc"]);

bool fout $[3]=\{$ true, true, true $\}$;

MeshVectorField bfield ( MODE_3D, fout, scale, magScale, bfieldfn);

field_extrpl_e bfldextrpl[6]=\{FIELD_ZERO, FIELD_ZERO,

FIELD_ZERO, FIELD_ZERO,

FIELD_ZERO, FIELD_ZERO \};

\} 


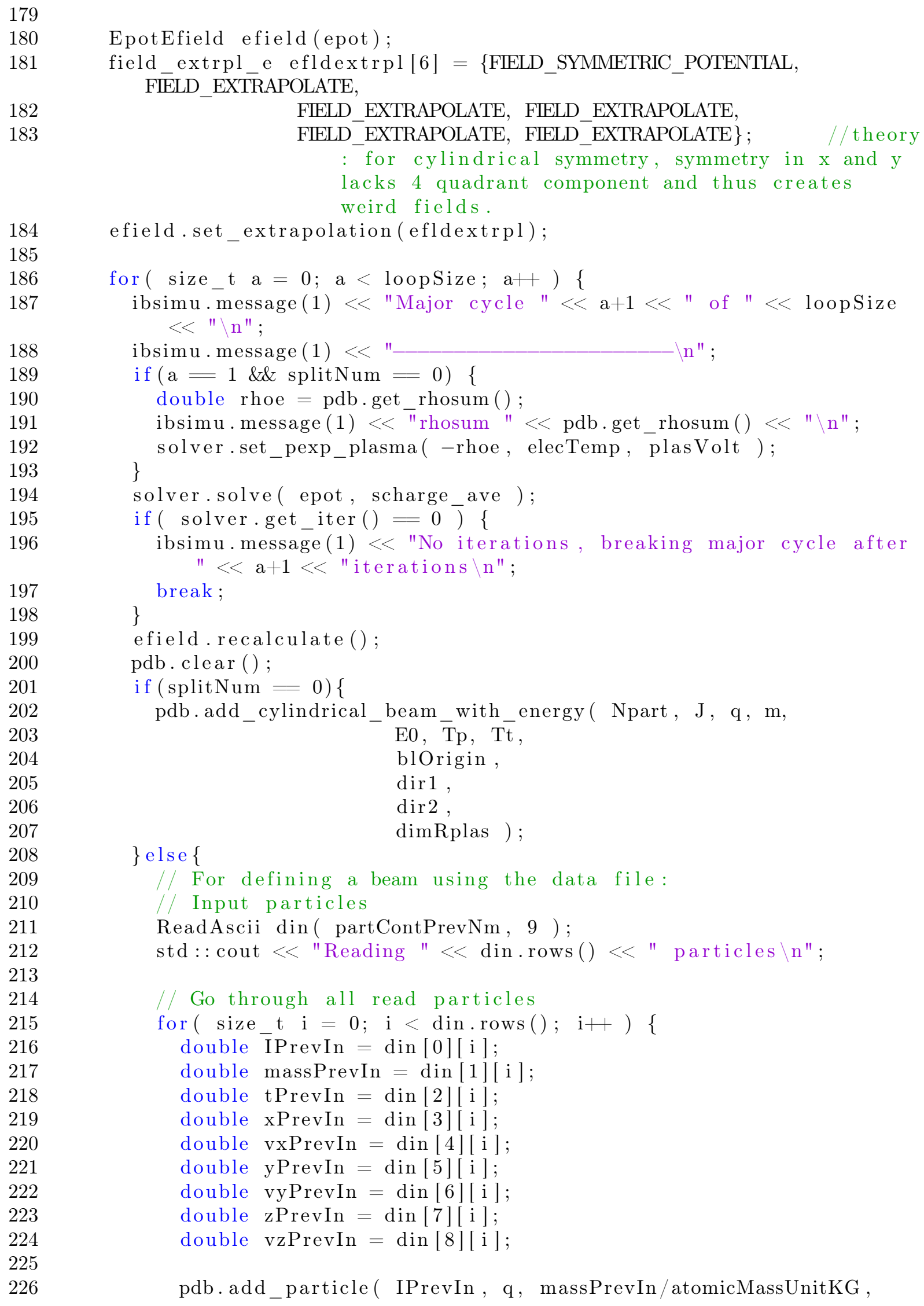


ParticleP3D (tPrevIn, xPrevIn, vxPrevIn, yPrevIn , vyPrevIn , zPrevIn , vzPrevIn ) ) ; \} 


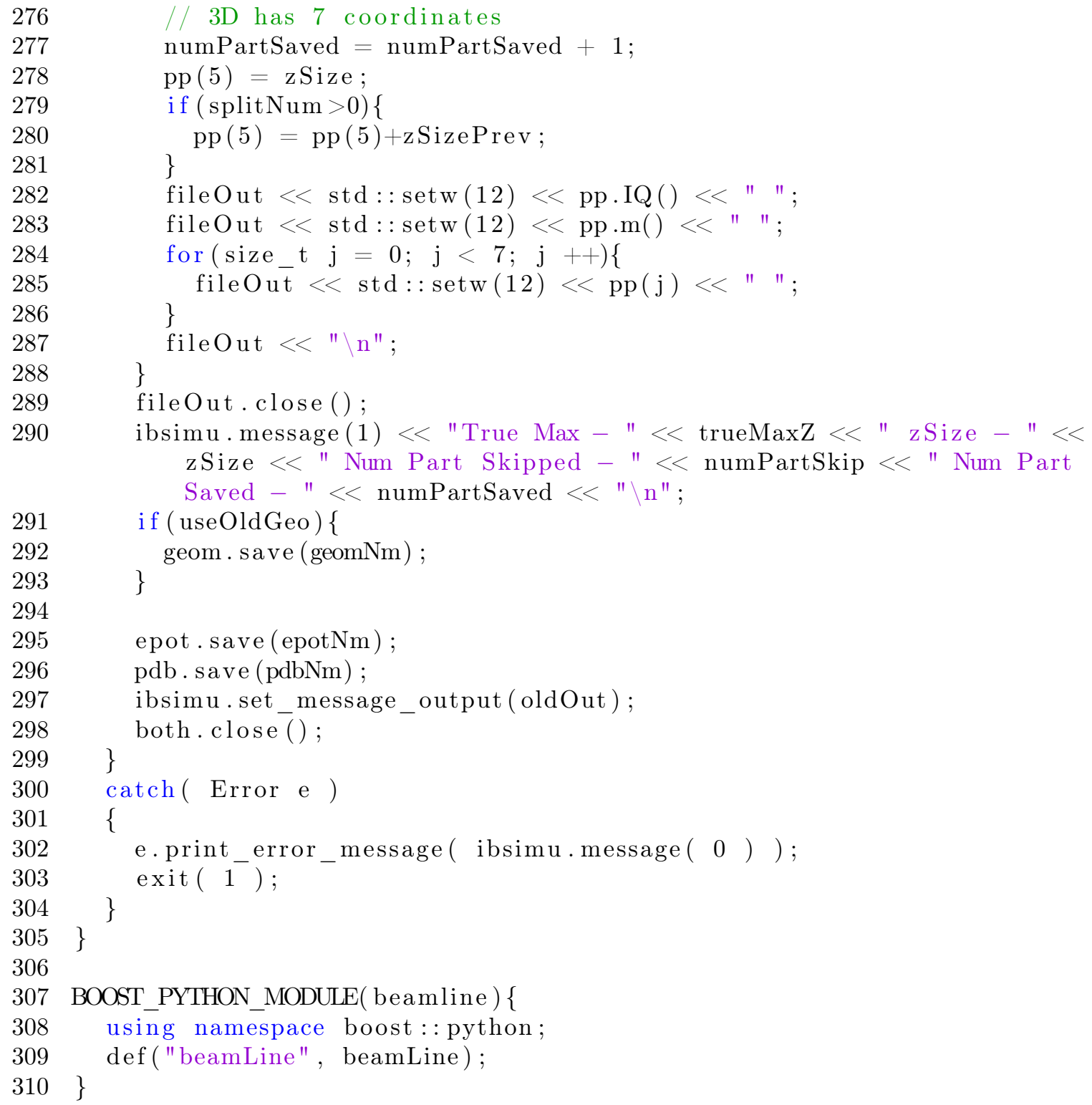

Listing 6: beamline.cpp

\section{A.2.6 beamDiag}

1 \#include $<$ fstream $>$

2 \#include <iomanip $>$

3 \#include $<$ limits $>$

4 \#include $<$ cmath $>$

5 \#include $<$ string $>$

6 \#include < boost/python.hpp>

7 \#include <boost/python/def.hpp>

8 \#include < boost/python/list.hpp>

9 \#include < boost/python/str.hpp>

10 \#include <boost/python/extract.hpp>

11 \#include < boost/numeric/conversion/cast.hpp> 


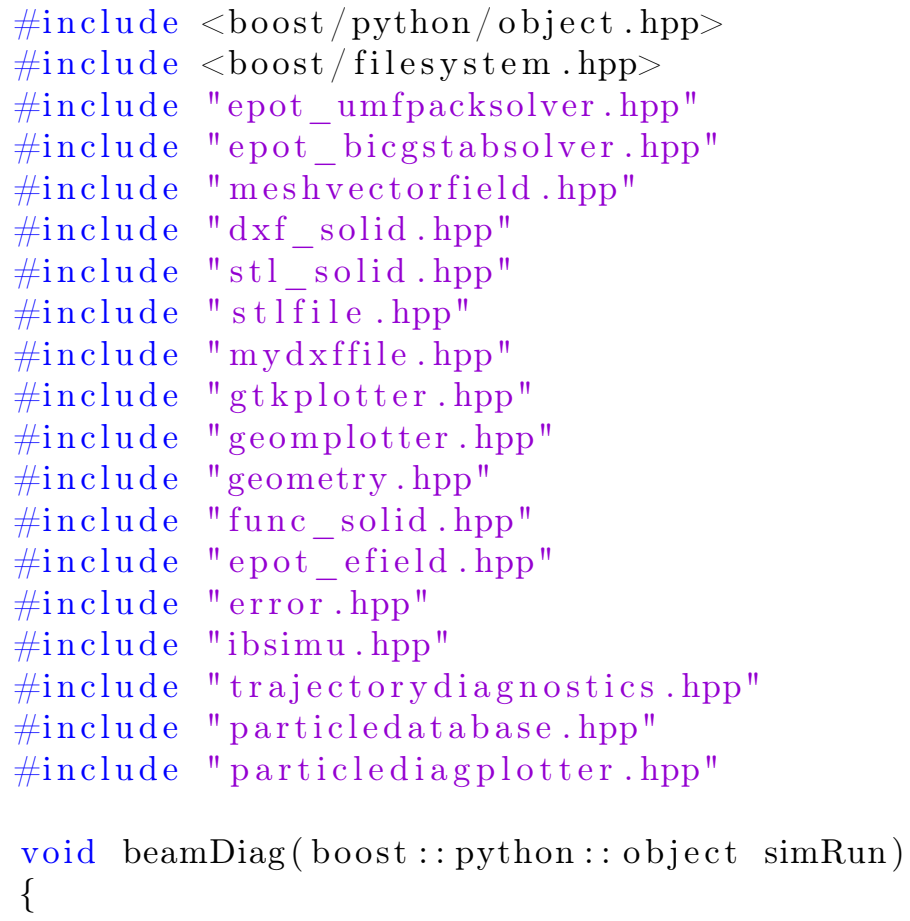


simRun . attr ("geom") [ splitNum ]));

Geometry geom( geoStream );

geom.build_surface ();

bool useMag $=$ boost : : python : : extract $<$ bool $>$ (simRun.attr ("useMag" $))$;

if ( useMag) \{

std:: string bfieldfn = boost:: python : : extract $<$ std : : string $>$ (simRun attr ("simVars") [ "magLoc"]);

bool fout $[3]=\{$ true, true, true $\}$;

MeshVectorField bfield( MODE_3D, fout, scale, magScale, bfieldfn);

field_extrpl_e bfldextrpl[6] $=\{$ FIELD_ZERO, FIELD_ZERO,

FIELD_ZERO, FIELD_ZERO,

FIELD_ZERO, FIELD_ZERO \};

\}

bfield.set_extrapolation( bfldextrpl);

std:: istringstream epoStream(boost : : python : : extract $<$ std : : string $>($

simRun.attr ("epot") [ splitNum]));

EpotField epot( epoStream, geom );

EpotEfield efield( epot );

field extrpl e efldextrpl[6]=\{FIELD_SYMMETRIC_POTENTIAL,

FIELD_EXTRAPOLATE,

efield.set_extrapolation(efldextrpl);

std: : istringstream pdbStream (boost: : python : : extract $<$ std $:$ : string $>($ simRun.attr ("pdb") [splitNum]));

ParticleDataBase3D pdb( pdbStream, geom );

79

MeshScalarField scharge(geom);

MeshScalarField tdens( geom );

pdb.build_trajectory_density_field( tdens );

double $z$ Level $=$ zMax - zLevelShift;

$/ /$ for (int $\mathrm{i}=0 ; \mathrm{i}<$ boost: : python: : len ( $\operatorname{simRun}$.attr ("geomItemAndVars")

["geomZpos"]); i ++$)\{$

86 // double zLevelitem = boost : : python: : extract $<$ double $>$ (simRun. attr (" geomItemAndVars") ["geomZpos "] [i ]);

$/ /$ double zLevel $=$ zLevelItem - zLevelShift;

for $($ int $\mathrm{j}=0 ; \mathrm{j}<\mathrm{i} ; \mathrm{j}++)\{$

zLevelItem = boost : : python : : extract $<$ double $>$ (simRun. attr $("$ geomItemAndVars") ["geomZpos "] [ j ] ) ; 


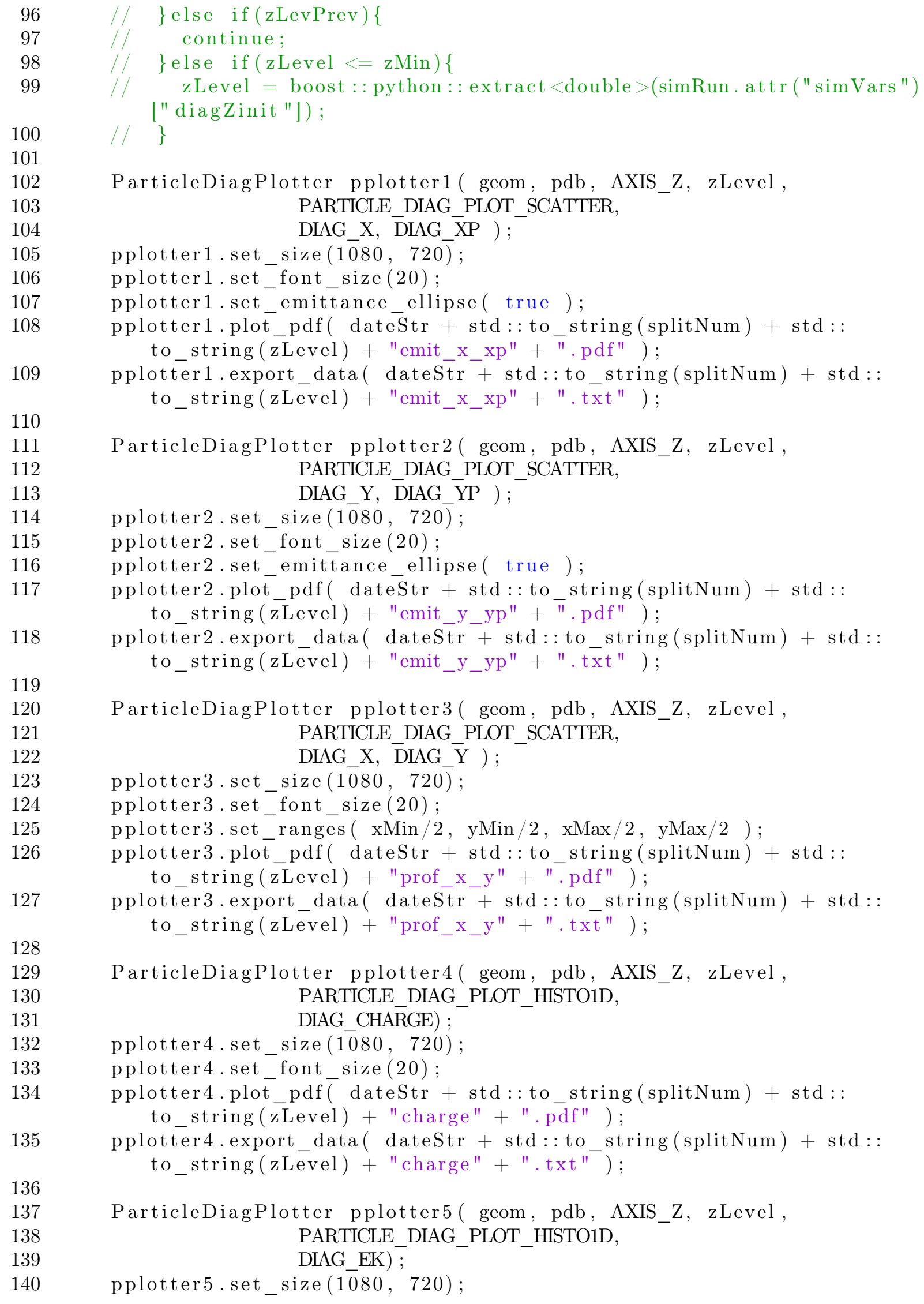

ParticleDiagPlotter pplotter1 ( geom, pdb, AXIS_Z, zLevel, PARTICLE_DIAG_PLOT_SCATTER, DIAG_X, DIAG_XP );

pplotter 1. set_size $(1 \overline{0} 80,720 \overline{)}$;

pplotter1.set_font_size $(20)$;

pplotter1.set_emittance_ellipse ( true );

pplotter1.plot_pdf( datestr + std:: to_string (splitNum) + std: :

to_string $(\overline{z L e v e l})+$ "emit_x_xp" $+\overline{\text { L }}$. pdf" );

pplotter 1.export_data ( dateStr__ std:: to_string ( $\operatorname{splitNum})+\operatorname{std}:$ :

to_string $($ zLevel $)+$ "emit_x_xp" + ". tx

ParticleDiagPlotter pplotter2( geom, pdb, AXIS_Z, zLevel, PARTICLE_DIAG_PLOT_SCATTER, DIAG_Y, DIAG_Y YP );

pplotter 2 .set_size $(1 \overline{0} 80,720)$;

pplotter2.set_font_size $(20)$;

pplotter2.set_emittance_ellipse ( true );

pplotter2.plot_pdf( datestr + std:: to_string (splitNum) + std:

to_string (zLevel) + "emit_y_yp" $+\overline{\text { zLepdf }}$ " );

pplotter2.export_data( dateStr + std:: to_string(

to_string $($ zLevel $)+$ "emit_y_yp" + ".txt" );

ParticleDiagPlotter pplotter3 ( geom, pdb, AXIS_Z, zLevel, PARTICLE_DIAG_PLOT_SCATTER,

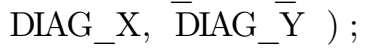

pplotter 3. set_size $(1 \overline{0} 80,720)$;

pplotter3.set_font_size $(20)$;

pplotter3.set_ranges $(x \operatorname{xin} / 2, \quad y M i n / 2, \quad x M a x / 2, \quad y M a x / 2)$;

pplotter3.plot_pdf( dateStr + std:: to_string(

to_string (zLevel) + "prof_x_y" + ".pdf" );

pplotter3.export_data( dateStr_ std: : to_string (

to_string (zLevel) + "prof_x_y" + ".txt" );

ParticleDiagPlotter pplotter4( geom, pdb, AXIS_Z, zLevel, PARTICLE_DIAG_PLOT_HISTO1D, DIAG_CHĀ $\bar{R} G \mathrm{E})$;

pplotter 4 .set_size $(1 \overline{0} 80,720)$;

pplotter 4 .set_font_size $(20)$;

pplotter4.plot_pdf( dateStr + std: : to_string (splitNum) + std : :

to_string (zLevel) + "charge" + ".p-pdf" );

pplotter4.export_data( dateStr + std: : to_string ( $\operatorname{splitNum)~+~std::~}$

to_string (zLevel) + "charge" + ".txt" );

ParticleDiagPlotter pplotter5( geom, pdb, AXIS_Z, zLevel, PARTICLE_DIAG_PLOT_HISTO1D, DIAG_EK) ;

pplotter 5 .set_size $(1 \overline{0} 80,720)$; 


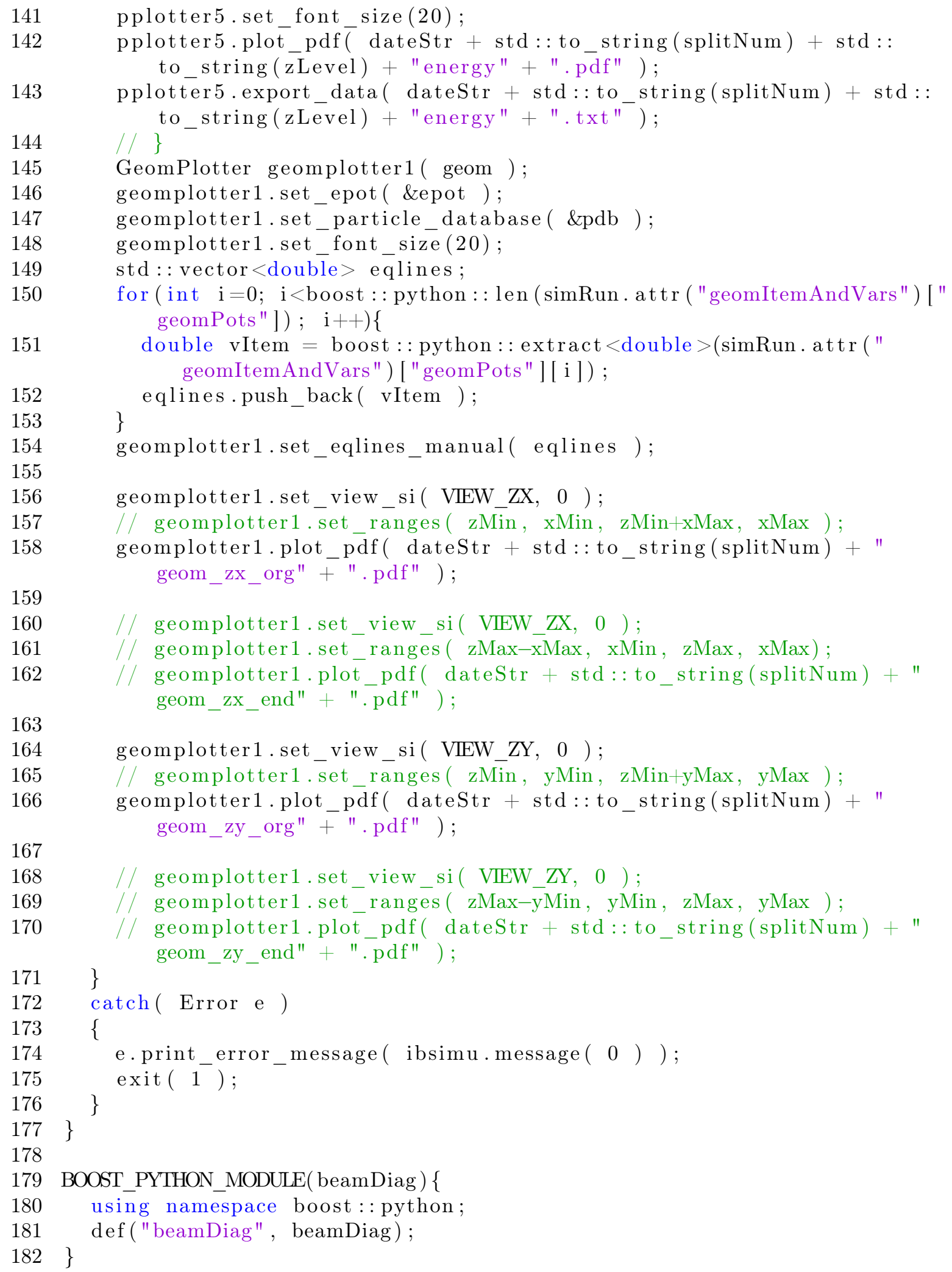

Listing 7: beamDiag.cpp 


\section{A.2.7 analysis}

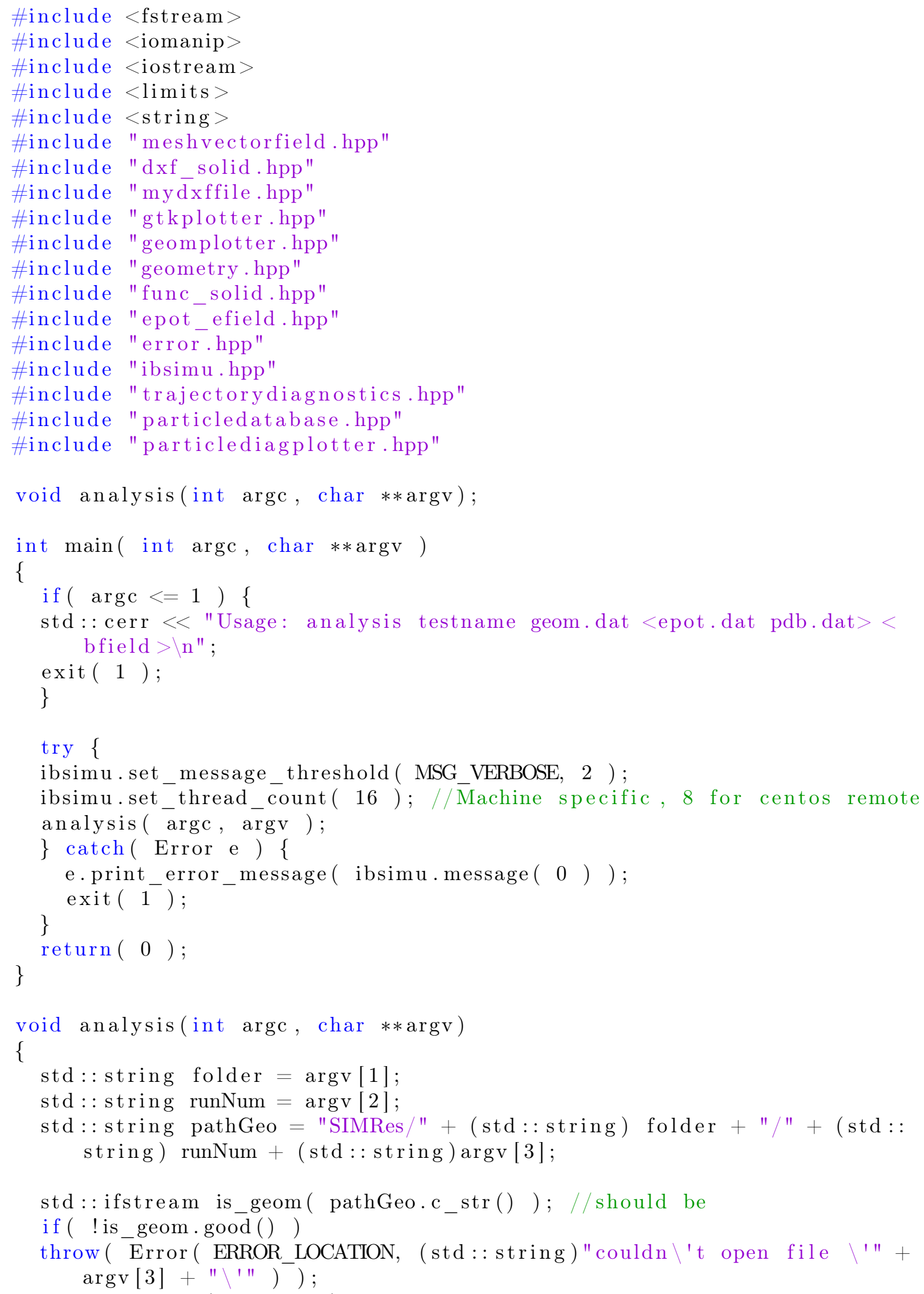




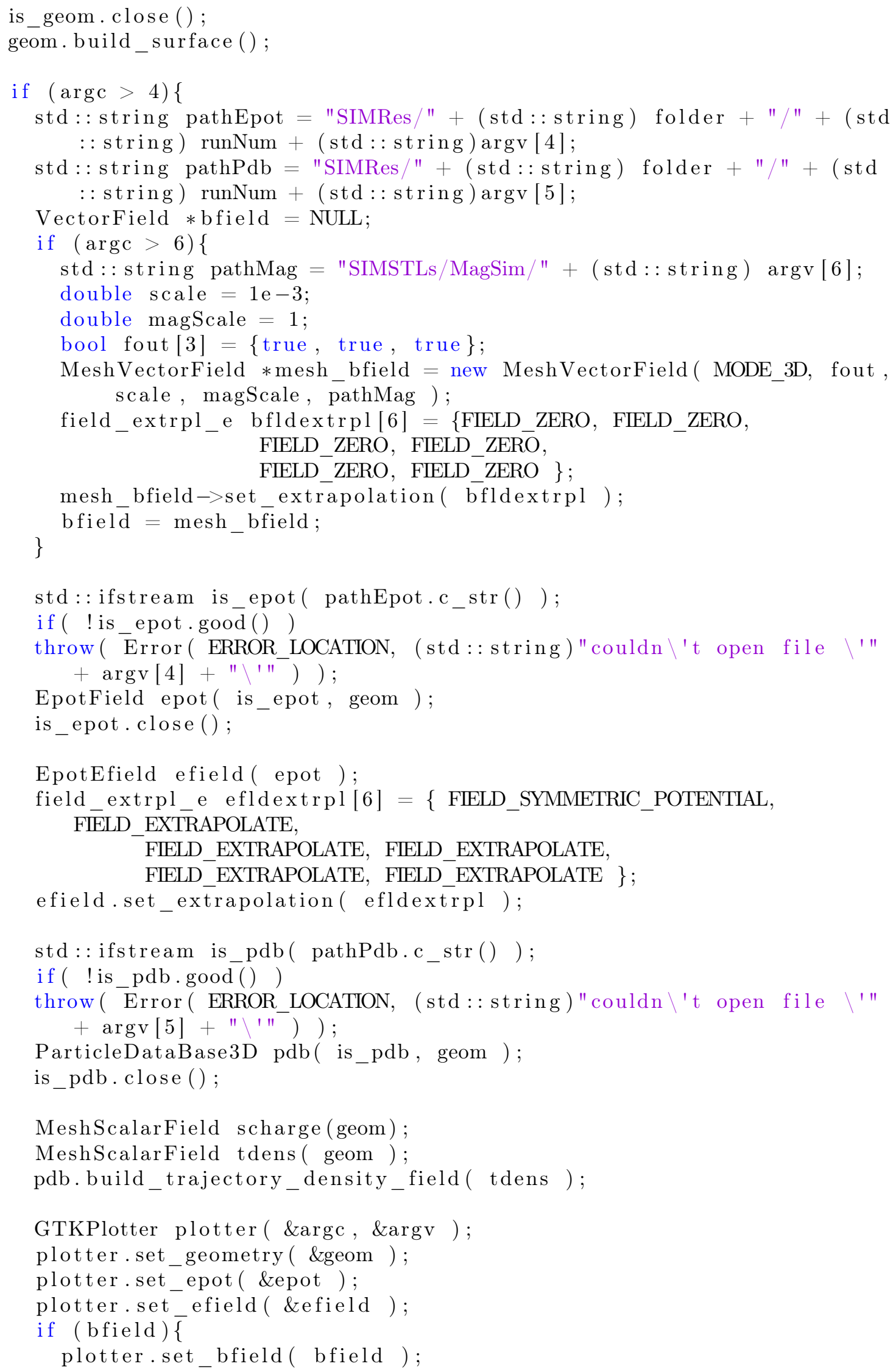




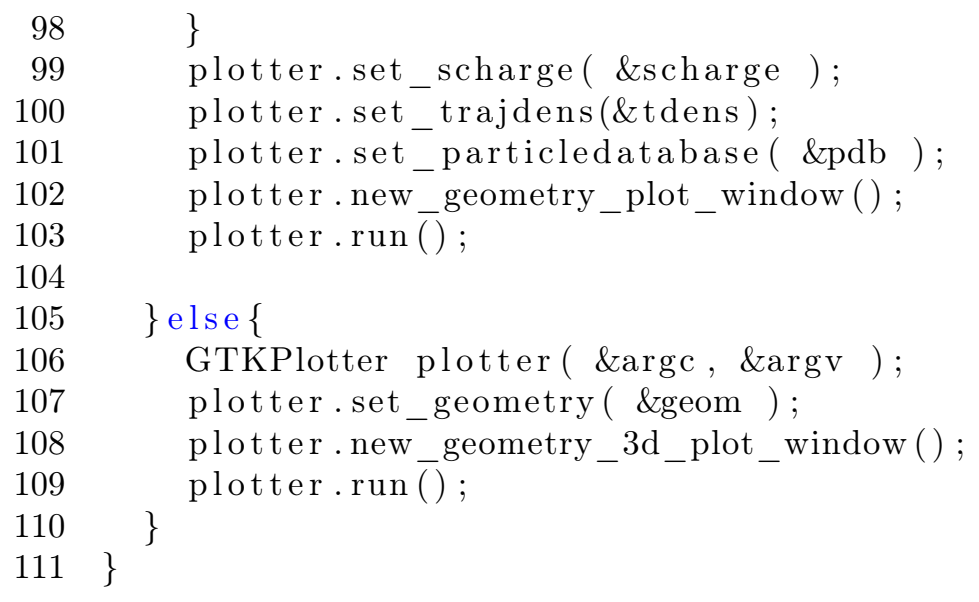

Listing 8: analysis.cpp

\section{A.3 Additional Calculation Code}

\section{A.3.1 simParamCalc}

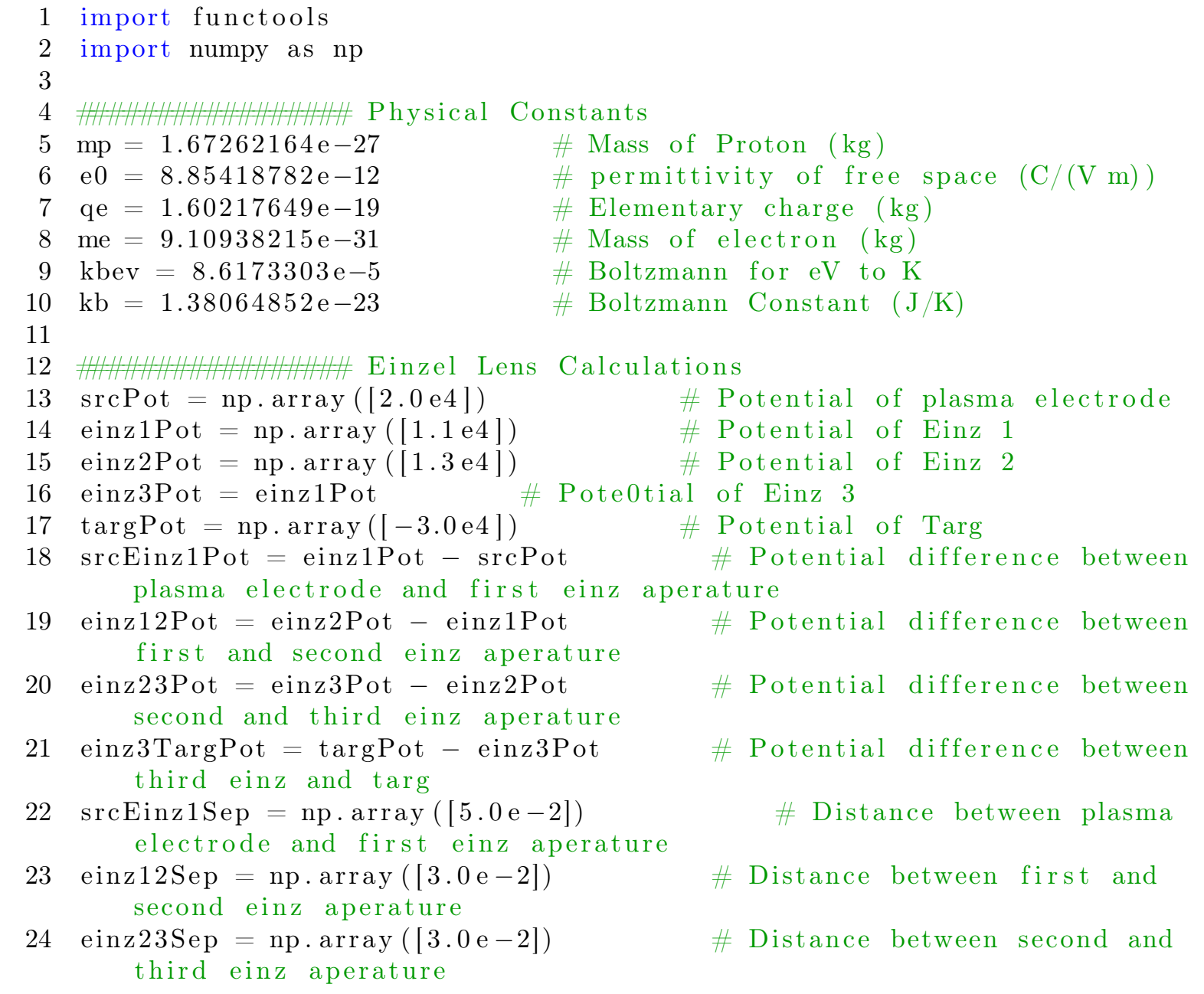




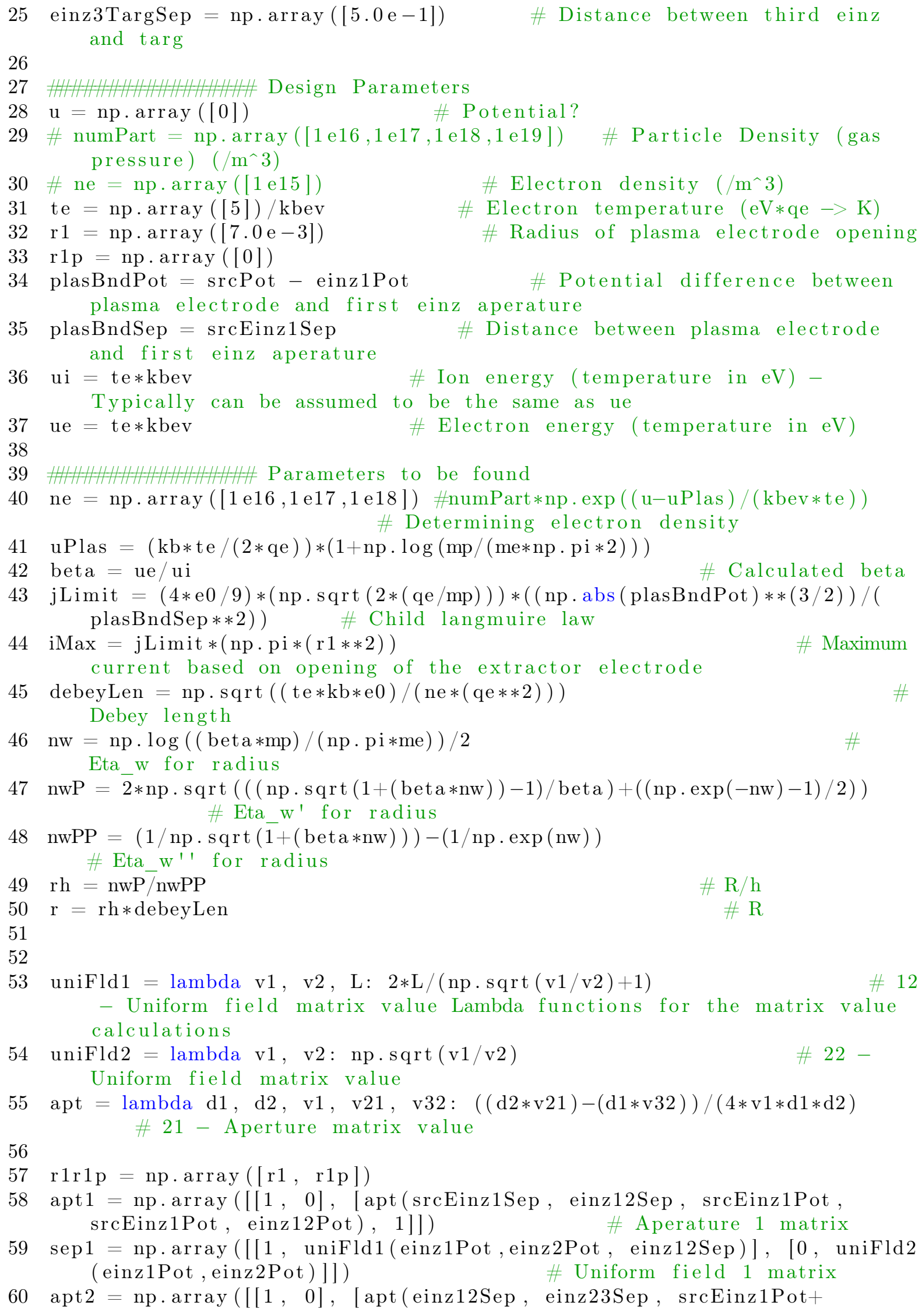




$$
\text { einz12Pot, einz12Pot, einz23Pot), 1]]) \# Aperture } 2
$$

matrix

61 sep2 = np.array $([[1$, uniFld1 (einz2Pot, einz3Pot, einz23Sep)], [0, uniFld2

(einz2Pot, einz3Pot)]]) \# Uniform field 2 matrix

62 apt $3=$ np.array $([[1,0], \quad[\operatorname{apt}(\operatorname{einz} 23 \mathrm{Sep}$, einz3TargSep, srcEinz1Pot+

einz12Pot+einz3Pot, einz23Pot, einz3TargPot), 1]]) \# Aperture 3

matrix

totMat = apt1@sep1@apt2@sep2@apt3 \# Einzel lens effect on trajectory is calculated by multiplying aperture, uniform field, aperture, uniform field, aperture

$64 \mathrm{r} 2 \mathrm{r} 2 \mathrm{p}=\mathrm{np} \cdot \operatorname{dot}($ totMat, $\operatorname{rr} 1 \mathrm{p}) \quad$ \# Determining the post-einzel lens trajectory

65 distTo2 $\mathrm{cm}=(2 \mathrm{e}-2-\mathrm{r} 2 \mathrm{r} 2 \mathrm{p}[0]) / \mathrm{r} 2 \mathrm{r} 2 \mathrm{p}[1] \quad \#$ Determining the distance to the size of the target (taken to have a radius of $2 \mathrm{~cm}$ )

66

67

68

69

70

print ('Number of Electrons $=$ ', ne, ' Current density $\left(\mathrm{A} / \mathrm{m}^{\wedge} 3\right)=$ ', jLimit, 'Max Current $(\mathrm{A})=$ ', iMax) print ('Debey Length $(\mathrm{m})=$ ', debeyLen, 'Circle Radius $=(\mathrm{m})$ ', $\mathrm{r}$ ) print ('(r2, r2p', r2r2p, ' distance to $2 \mathrm{~cm}=1$, distTo2 cm) print ('Plasma Potential = ', uPlas)

Listing 9: simParamCalc.py

\section{A.3.2 wallThickCalc}

1

2

2

3

4

4

5

6

12

13

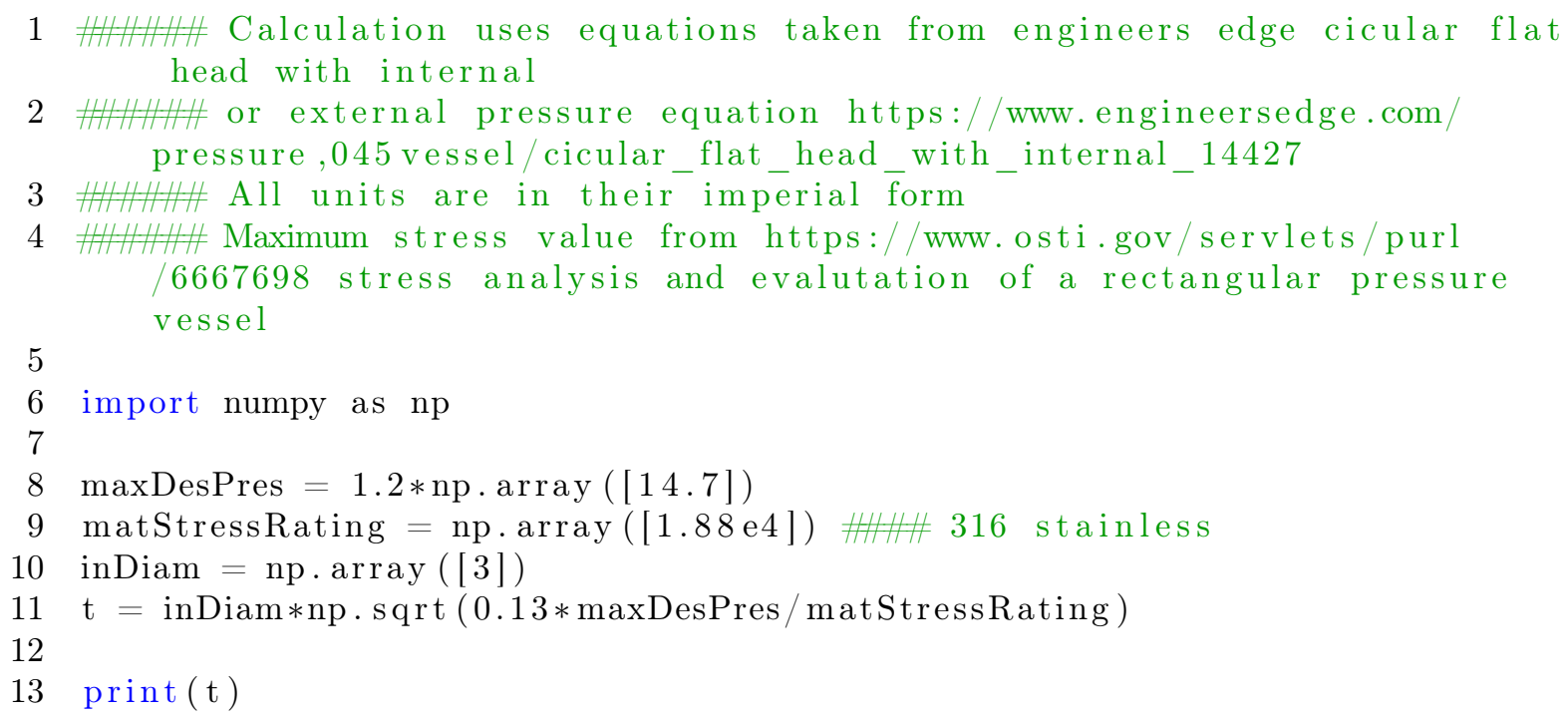

8 maxDesPres $=1.2 *$ np. array $([14.7])$

9 matStressRating $=$ np.array $([1.88 \mathrm{e} 4])$ \#\#\#\#316 stainless

10 inDiam $=$ np. array $([3])$

$11 \mathrm{t}=\operatorname{inDiam} * \mathrm{np} . \operatorname{sqrt}(0.13 *$ maxDesPres/matStressRating $)$

Listing 10: wallThickCalc.py

\section{A.3.3 geomPrepManDef}

1 \#include $<$ fstream $>$

2 \#include <iomanip $>$

3 \#include $<$ limits $>$

4 \#include $<$ string $>$

5 \#include < boost/python.hpp> 


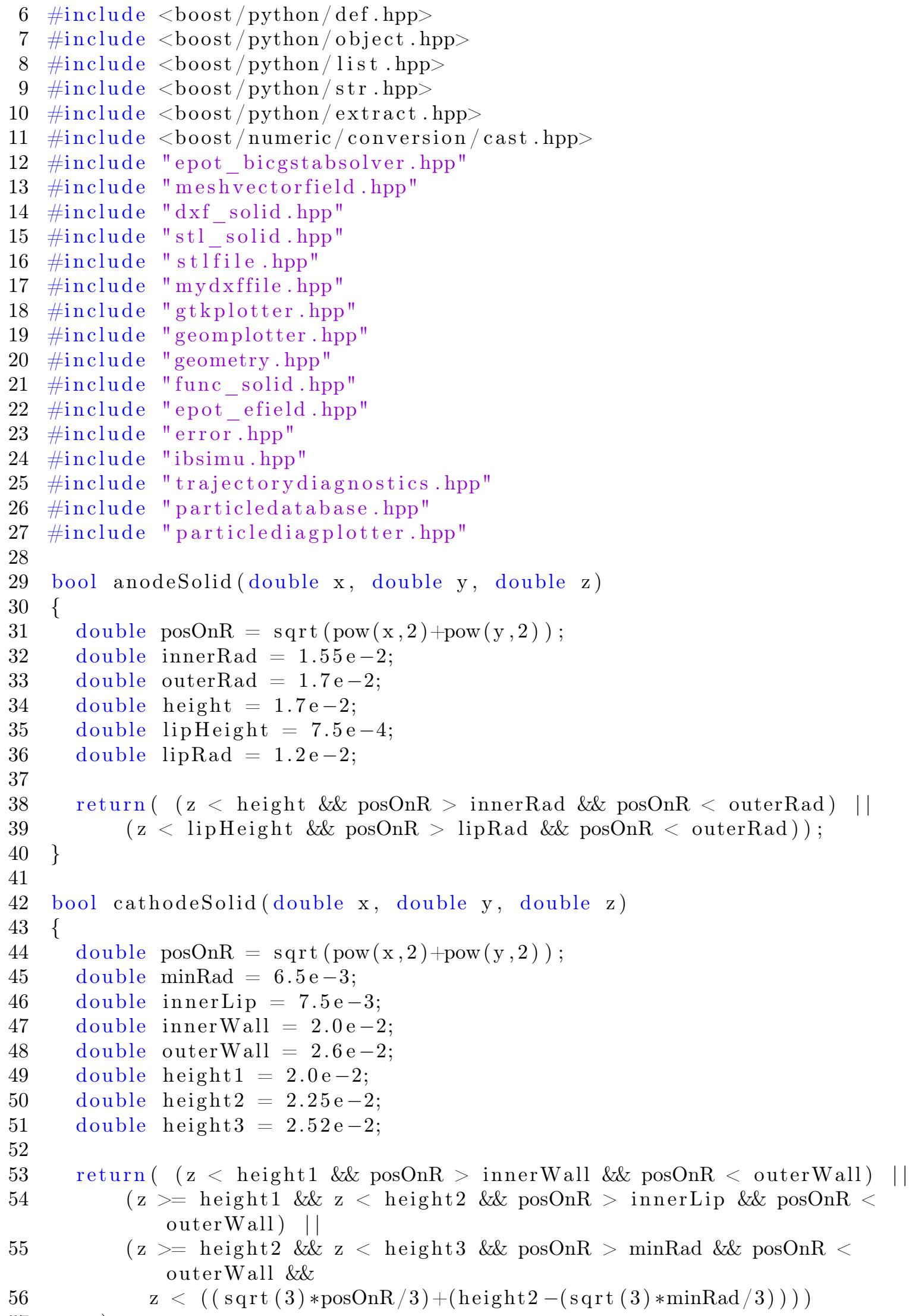




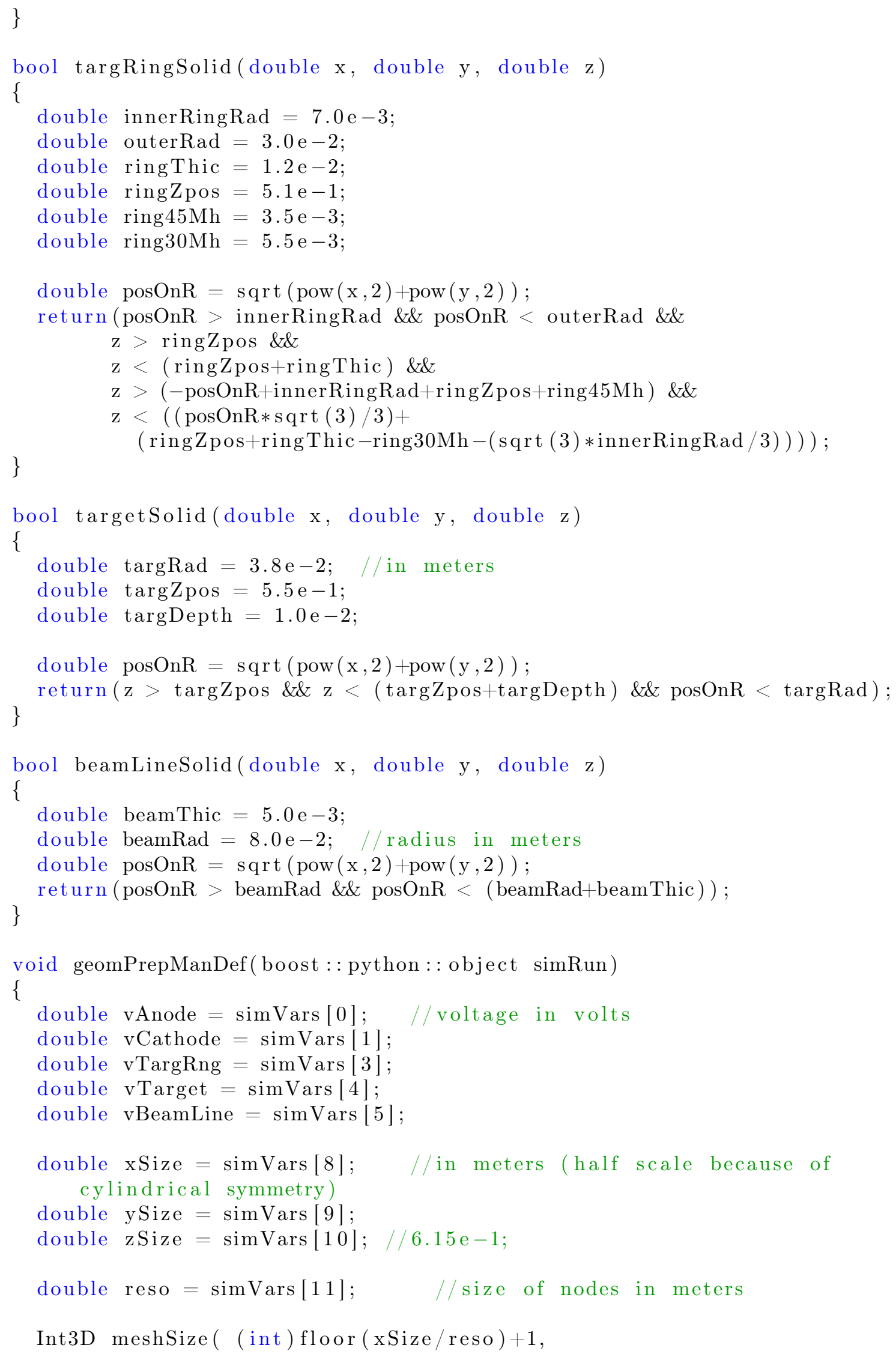




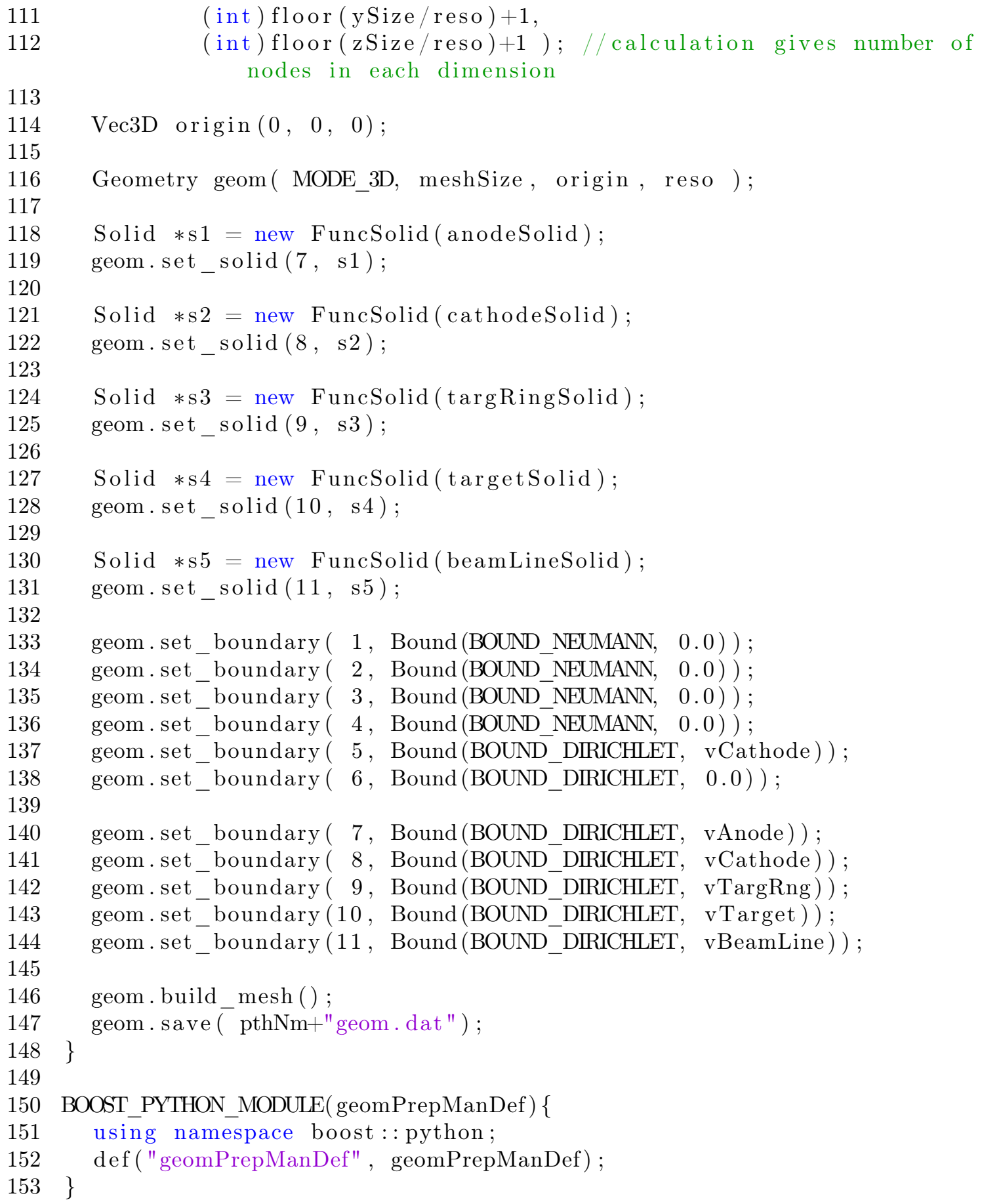

149

150 151 152 153

Listing 11: geomPrepManDef.cpp 